\title{
23. QUATERNARY RADIOLARIANS FROM THE MOUTH OF THE GULF OF CALIFORNIA, DEEP SEA DRILLING PROJECT LEG $65^{1}$
}

\author{
Richard N. Benson, Delaware Geological Survey, University of Delaware, Newark, Delaware
}

\section{INTRODUCTION}

I recovered well-preserved radiolarian assemblages from the Quaternary sediments drilled at all four sites at the mouth of the Gulf of California during Leg 65 (Fig. 1). The sites, with positions and water depths averaged for all hole locations per site, are

Site $482-22^{\circ} 47.4^{\prime} \mathrm{N}, 107^{\circ} 59.6^{\prime} \mathrm{W}$; water depth, 3022 meters.

Site $483-22^{\circ} 53.0^{\prime} \mathrm{N}, 108^{\circ} 44.8^{\prime} \mathrm{W}$; water depth, 3070 meters.

Site $484-23^{\circ} 11.2^{\prime} \mathrm{N}, 108^{\circ} 23.6^{\prime} \mathrm{W}$; water depth, 2887 meters.

Site $485-22^{\circ} 44.9^{\prime} \mathrm{N}, 107^{\circ} 54.2^{\prime} \mathrm{W}$; water depth, 2981 meters.

The nearly 200 taxa I identified are listed alphabetically in the systematic reference list. The only reliable radiolarian biostratigraphic datum determined for the Quaternary sedimentary section is the highest occurrence of Axoprunum angelinum (Hays) at Sites 483, 484 , and 485 .

\section{THE RADIOLARIAN ASSEMBLAGE}

With some additions, the species identified from Leg 65 are the same I described in earlier studies of Holocene sediments from the Gulf of California (Benson, $1964,1966)$. The major differences between the Holocene and older assembiages are in the relative abundances of individual species. I could not determine whether these differences in abundance reflect environmental conditions, preservation, or both.

\section{Holocene Radiolarians from the Gulf of California}

In an earlier study (Benson, 1966), I found radiolarians in the Recent sediments at 26 of 28 stations distributed throughout the Gulf of California (Fig. 2). At the time, I concluded that the Holocene assemblage was derived primarily from equatorial Pacific waters. From an examination of recent literature on the distribution of modern radiolarians, particularly in the eastern $\mathrm{Pa}$ cific but also in high as well as intermediate and low latitudes (Riedel, 1958; Nigrini, 1967, 1968, 1970; Casey, 1971, 1977; Ling et al., 1971; Kling, 1973, 1977; Molina-Cruz, 1977; Nigrini and Moore, 1979), I conclude that the assemblage is primarily tropical to subtropical but with contributions of cooler water species from the California Current System.

\footnotetext{
${ }^{1}$ Lewis, B. T. R., Robinson, P., et al., Init. Repts, DSDP, 65: Washington (U.S, Govt. Printing Office).
}

Table 1 lists the dominant members of the Holocene assemblage in the Gulf, as determined by averaging the percentages for each species at each station. The quantitative methods used in my earlier research to obtain the percentages are as follows. I first scanned all strewn slides prepared from the $\mathrm{HCl}$-insoluble, clay-free residues of sediments from the 28 sampling stations in order to determine the occurrence or nonoccurrence of each species. Next, I counted 500 tests for each station, preliminary counts of 1000 having shown no significant differences from the 500 count in relative frequencies of each species at a station. The slide with the greatest concentration of tests was chosen for purposes of counting. In order to include as many variations in test density as possible on the slide, I made a diagonal traverse across the $22 \mathrm{~mm} \times 44 \mathrm{~mm}$ area under the cover glass. Six of the 28 stations yielded total populations of less than 500. The counts for each species at each station were converted into percentages.

Species that show cooler water affinities and that are probably, at least in part, from the California Current System include Hexacontium enthacanthum, Stylochlamydium venustum, Lithomelissa hystrix, Larcopyle bütschlii, Lithelius minor, Pterocorys minythorax, $\mathrm{Hel}$ otholus histricosa group, Actinosphaera cristata(?), and Theocalyptra davisiana s.l. The influence of the California Current System is evident in Bé's (1977) map of the major faunal provinces of living planktonic foraminifers. The current carries a higher latitude (Transition Zone) assemblage southward along the west coast of Baja California. The intrusion of the cool water current into subtropical and tropical waters causes three faunal provinces to converge at a point offshore from southern Baja California, namely, the Transition Zone, Subtropical Faunal Province, and Tropical Faunal Province. Likewise, the Holocene radiolarian assemblage within and at the mouth of the Gulf of California represents a mixing of species from similar latitudinally defined radiolarian provinces.

I did take into consideration the commingling in the sediments of tests of species occupying overlying water masses which are vertically separated or distinct from one another. For example, Kling (1977) attributes the decrease in abundance of Theocalyptra davisiana davisiana, which occupies the temperate and polar regions of most oceans, from common in a core from the Santa Monica Basin to rare in one from the Santa Barbara Basin, to the fact that this species is restricted to the subsill depths of the Santa Monica Basin. The sill depth of the Santa Barbara Basin is 260 meters shallower. Petru- 


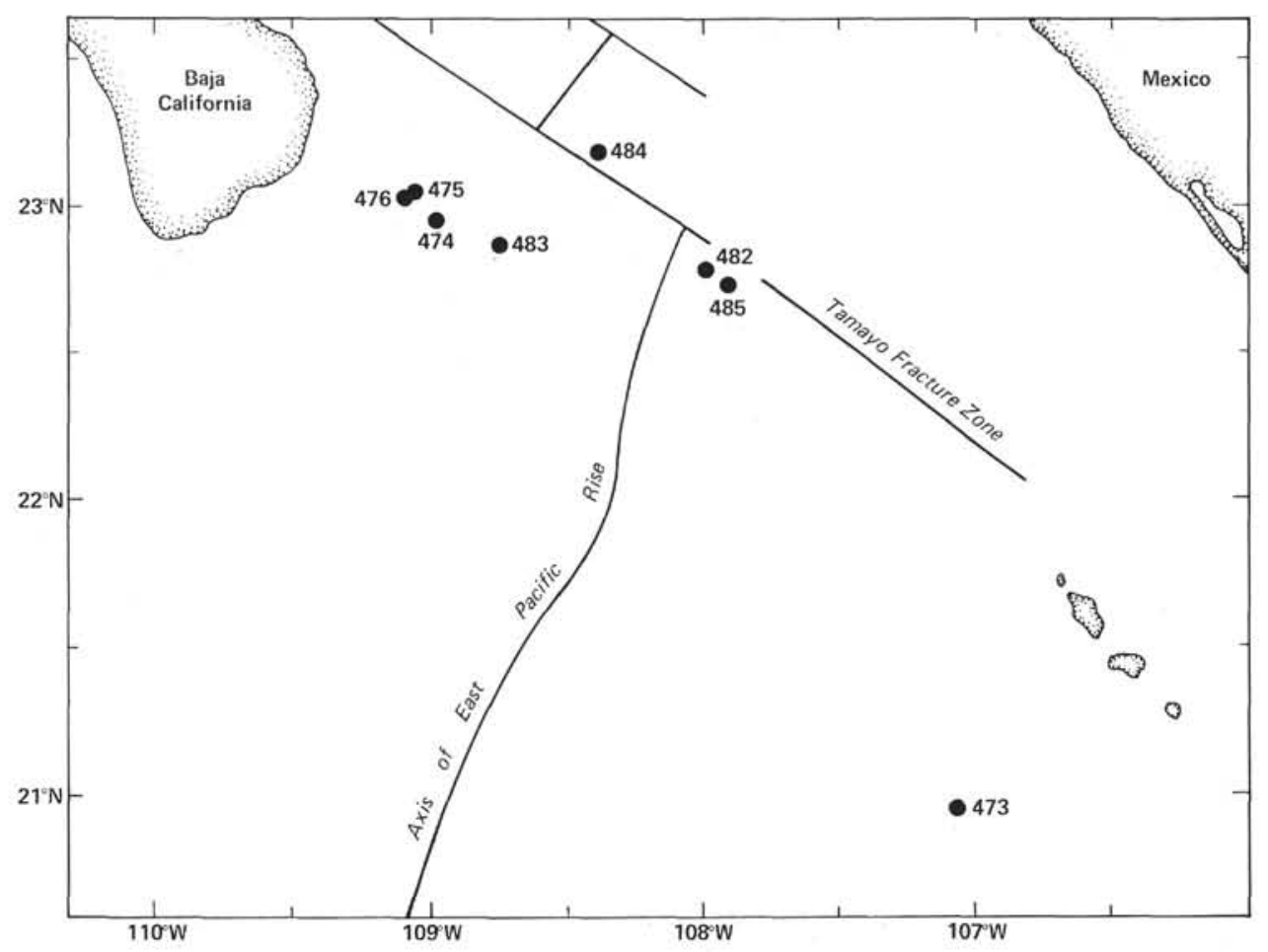

Figure 1. DSDP drilling sites at the mouth of the Gulf of California. Site 473 was drilled on Leg 63, Sites 474 through 476 were drilled on Leg 64, and Sites 482 through 485 were drilled on Leg 65.

shevskaya and Björklund (1974) also note that this species (their Diplocyclas davisiana) is associated with deep water, being common in deep water and rare in shallow water sediments of the Norwegian-Greenland seas. In the Gulf of California, T. davisiana s.l. (mostly $T$. davisiana davisiana but also $T$. davisiana cornutoides in smaller numbers) is more abundant in the deeper water sediments. $T$. davisiana davisiana is also a quantitatively important member of the Quaternary assemblage at Leg 65 sites, which are in relatively deep water. Figure 3 illustrates the strong correlation $(r=0.90)$ between water depth and the relative percentage of this species in each Holocene sample from the gulf (Benson, 1966) and in presumably Holocene samples from Sites 482,483 , and 485. Theocalyptra davisiana s.1. is probably representative of faunas living in submerged, colder water masses that contribute to the overall assemblage in the sediments of the Gulf of California.

\section{The Quaternary Assemblage at Leg 65 Sites}

Tables 2 through 5 list the abundance and degree of preservation of radiolarians in samples examined from Leg 65 sites. Data are shown graphically in Figure 4.

The overall assemblage is approximately the same at all four sites, dominant species being among the spumellines Tetrapyle octacantha group, Phorticium pylonium group, Actinosphaera cristata(?), Hexacontium enthacanthum, Druppatractus variabilis, Thecosphaera spp., Lithelius minor, Lithelius(?) sp., and several spongodiscids; among the nassellines Theocalyptra davisiana davisiana, Botryostrobus auritus-australis group, B. aquilonaris, and Lamprocyclas maritalis martialis. In samples with abundant radiolarians, more than 100 spe- cies are present. The number of nasselline species generally exceeds that of spumelline species, but the number of spumelline tests exceeds the number of nasselline tests.

In addition to those already noted, many other species are persistent and quantitatively important in the Quaternary section. Spumellines include Acrosphaera murrayana, Actinomma antarcticum, A. leptodermum, A. medianum, Hexacontium heteracantha, Druppatractus irregularis, Amphisphaera cristata, Xiphatractus cronos, $X$. pluto, Ommatartus tetrathalamus, Amphirhopalum ypsilon, Dictyocoryne profunda, D. truncatum, Euchitonia elegans, Euchitonia sp. cf. E. furcata, Hymeniastrum euclidis, Stylochlamydium asteriscus group, and $S$. venustum group. Nassellines include Liriospyris reticulata, Lithomelissa monoceras, Dictyophimus crisiae, Carpocanistrum sp. A, C. petalospyris group, Cornutella profunda, Lamprocyclas maritalis polypora, Anthocyrtidium ophirense, Theocorythium trachelium trachelium, Lamprocyrtis nigriniae, L.(?) hannai, Pterocorys minythorax, Theocalyptra davisiana cornutoides, Eucyrtidium hexagonatum, Siphocampe lineata group, and Phormostichoartus corbula.

I found no significant changes in the assemblage with depth at any site. As in the case of the Holocene assemblage, the mouth of the Gulf of California was apparently a region where species from both lower and higher latitudes mixed during the Pleistocene. There was no domination by a strictly cold water assemblage, at least not for sufficient time to have left a record which could be detected with the sample spacing used in this study. Statistical analysis of data from more closely spaced samples at each site may reveal more subtle 


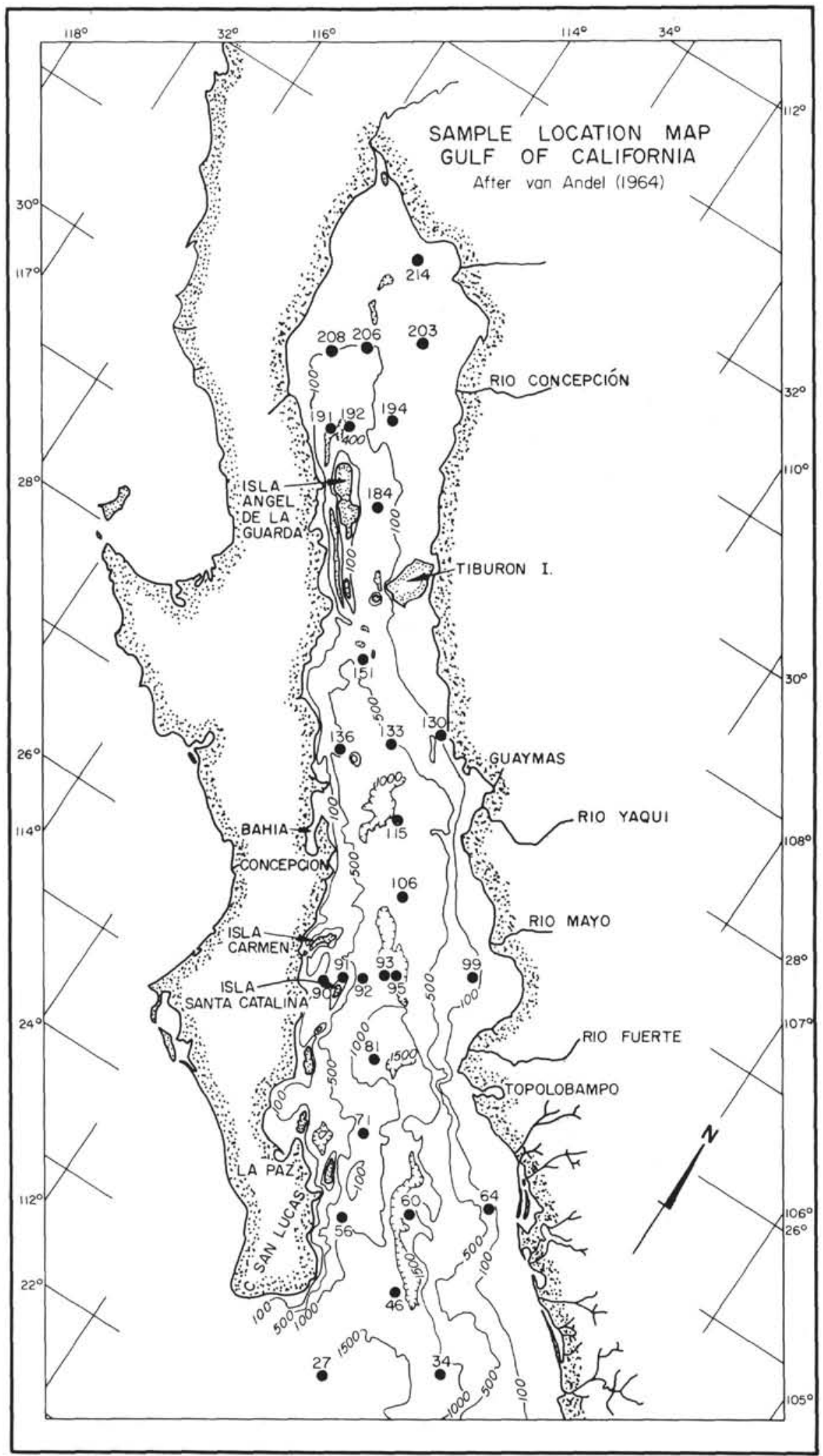

Figure 2. Sample location map, Gulf of California (from Benson, 1966). Numbers refer to cores collected by G. A. Rusnak during the Vermilion Sea Expedition in 1959. (Depth contours in fathoms.) 
Table 1. Relative abundances of dominant Holocene radiolarians in the Gulf of California (after Benson, 1966).

\begin{tabular}{|c|c|c|}
\hline Taxon & $\begin{array}{c}\text { Av. } \% \\
\text { Per Station } \\
\text { (26 stations) }\end{array}$ & $\begin{array}{c}\text { Stations } \\
\text { Where } \\
\text { Present } \\
\text { (out of 26) }\end{array}$ \\
\hline Tetrapyle octacantha group & 6.8 & 26 \\
\hline Phorticium pylonium group & 6.1 & 26 \\
\hline Druppatractus variabilis & 4.6 & 25 \\
\hline Hexacontium enthacanthum & 3.9 & 25 \\
\hline Eucyrtidium hexagonatum & 3.8 & 26 \\
\hline Stylochlamydium venustum/S. asteriscus & 3.8 & 26 \\
\hline Lithomelissa hystrix & 2.7 & 23 \\
\hline Spirocyrtis scalaris/S. subscalaris & 2.4 & 25 \\
\hline Larcopyle butschlii & 2.3 & 24 \\
\hline Lithelius minor & 2.1 & 25 \\
\hline Hexapyle dodecantha & 1.8 & 26 \\
\hline Ommatartus tetrathalamus & 1.7 & 26 \\
\hline Pterocorys minythorax $/ P$. zancleus & 1.7 & 21 \\
\hline Euchitonia sp. cf. E. furcata & 1.6 & 26 \\
\hline Lithomelissa monoceras & 1.5 & 25 \\
\hline Druppatractus irregularis & 1.5 & 23 \\
\hline Plectacantha oikiskos & 1.4 & 16 \\
\hline Helotholus histricosa group & 1.4 & 22 \\
\hline Pseudocubus obeliscus & 1.4 & 23 \\
\hline Actinosphaera cristata(?) & 1.2 & 25 \\
\hline Pterocanium bicorne(?) & 1.2 & 23 \\
\hline Theocalyptra davisiana s. I. & 1.0 & 18 \\
\hline Spongodiscus biconcavus & 1.0 & 26 \\
\hline Theopilium tricostatum & 1.0 & 23 \\
\hline Actinomma antaracticum & 0.9 & 19 \\
\hline Hexacontium laevigatum & 0.8 & 23 \\
\hline Plagiacantha(?) panarium & 0.8 & 17 \\
\hline Anomalacantha dentata & 0.7 & 20 \\
\hline
\end{tabular}

changes related to fluctuations of sea surface temperature during the Pleistocene.

Throughout the Pleistocene section, the planktonic foraminiferal data show the same lack of domination by species from any one of the three major modern faunal provinces which converge at a point off southern Baja California (Bé, 1977). Using my shipboard identifications, I constructed Table 6, which shows that in those samples from Site 483 with common to abundant foraminifers, the dominant species represent all three provinces. Throughout the Pleistocene, the California Current System was active at least as far south as the mouth of the Gulf of California, transporting large populations of such higher latitude species as Globigerina bulloides and Globoquadrina pachyderma to a subtropical to tropical region dominated by $G$. dutertrei, Globigerinoides ruber, G. sacculifer, Globorotalia menardii, and Pulleniatina obliquiloculata.

\section{BIOSTRATIGRAPHY}

\section{Quaternary Radiolarian Zonations and Datum Levels}

In the study of Leg 65 samples, I attempted to apply Quaternary radiolarian zonations and datum levels used in both equatorial (Nigrini, 1971; Dinkelman, 1973; Johnson and Knoll, 1975) and higher latitude studies (Hays, 1970; Kling, 1973). The absence or scarcity of the marker species Pterocanium prismatium, Theocorythium vetulum, Anthocyrtidium angulare, Collosphaera tuberosa, and Buccinosphaera invaginata precluded use of Nigrini's (1971) fourfold zonation of the

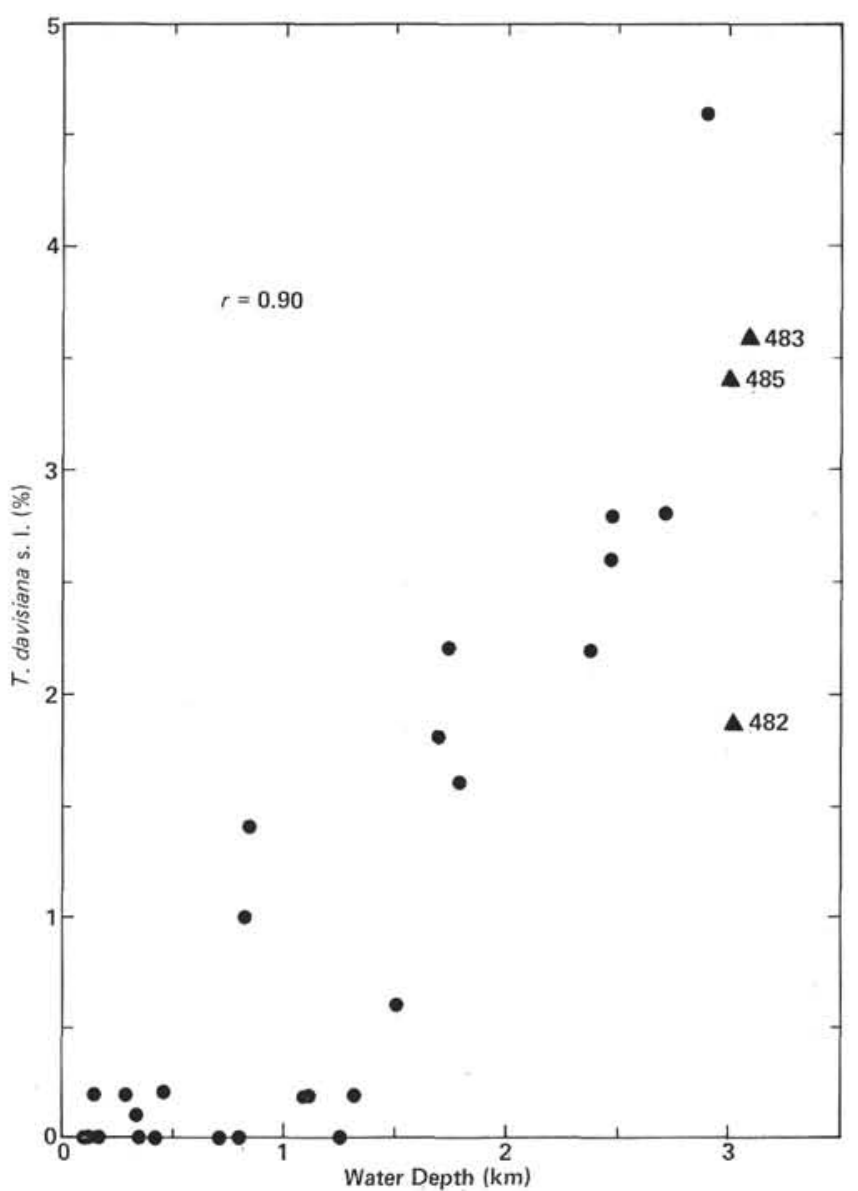

Figure 3. Relative percentage of Theocalyptra davisiana s.1. in Holocene radiolarian assemblages vs, water depth in the Gulf of California. (Closed circles = sampling stations of Benson (1966); closed triangles $=$ Leg 65 sites; $r$-correlation coefficient.)

Quaternary in equatorial Pacific sediments. The highest occurrence of Axoprunum angelium (= Stylatractus universus) is the only datum level I determined with any confidence. Sediments containing radiolarians above this level could be assigned to Kling's (1973) Artostrobium miralestense (= Botryostrobus aquilonaris) Zone and those below to his Axoprunum angelinum Zone ( $=$ S. universus Zone of Hays, 1970). Because of the absence of such marker species as Eucyrtidium matuyamai and Lamprocyrtis heteroporos, I could determine neither the base of the latter zone nor the presence of the underlying E. matuyamai Zone as defined by Hays (1970) or Kling (1973).

The need to determine rates of sediment accumulation at DSDP sites forces biostratigraphers to emphasize the importance of the ages in years of significant paleontological datum levels discovered in the sedimentary sections cored. Table 7 summarizes the estimated ages for several levels in the Quaternary. The reader should consult the references given in the table in order to assess the validity of the ages.

Data from Leg 65 support Hays and Shackleton's (1976) conclusion that the extinction level of $A$. angelinum $(=S$. universus) was globally synchronous at about $0.41 \pm 0.005 \mathrm{Ma}$. At Sites 483,484 , and 485 , this 
Table 2. Radiolarians at Site 482.

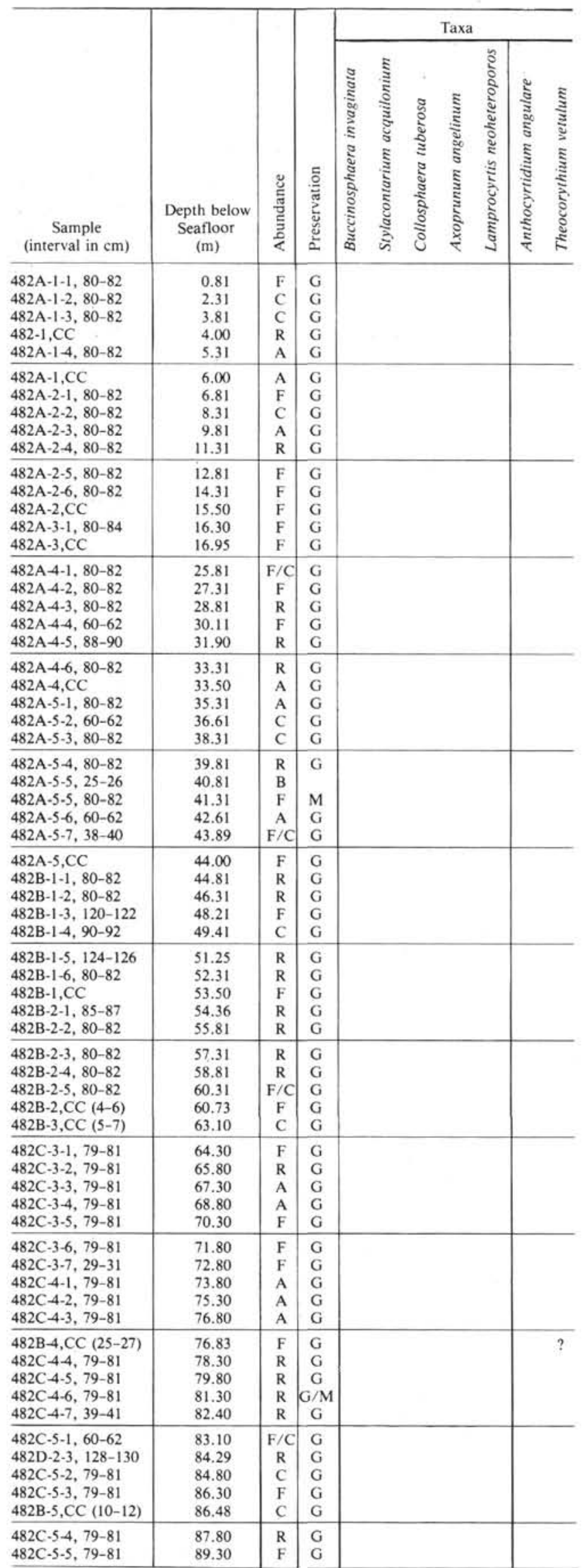

Table 2. (Continued).

\begin{tabular}{|c|c|c|c|c|c|c|c|}
\hline \multirow[b]{2}{*}{$\begin{array}{c}\text { Sample } \\
\text { (interval in cm) }\end{array}$} & \multirow[b]{2}{*}{$\begin{array}{l}\text { Depth below } \\
\text { Seafloor } \\
\text { (m) }\end{array}$} & \multirow[b]{2}{*}{ 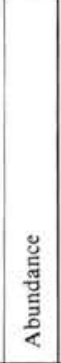 } & \multirow[b]{2}{*}{ 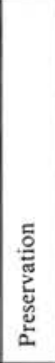 } & \multicolumn{4}{|c|}{ Taxa } \\
\hline & & & & 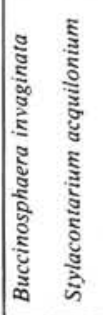 & 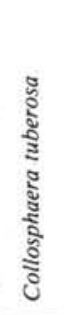 & 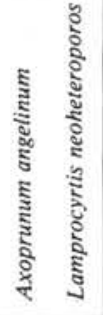 & 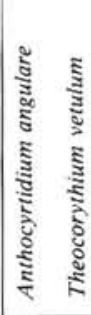 \\
\hline $\begin{array}{l}482 C-5-6,79-81 \\
482 C-5-7,79-81 \\
482 C-5, C C(8-10)\end{array}$ & $\begin{array}{l}90.80 \\
92.00 \\
92.00\end{array}$ & \begin{tabular}{|c|}
$R$ \\
$R / F$ \\
$R / F$ \\
\end{tabular} & $\begin{array}{l}\text { G } \\
\text { G } \\
\text { G } \\
\end{array}$ & & & & \\
\hline $\begin{array}{l}482 \mathrm{~B}-6-1,80-82 \\
482 \mathrm{~B}-6-2,92-94 \\
482 \mathrm{~B}-6-3,80-82 \\
482 \mathrm{~B}-6-4,80-82 \\
482 \mathrm{~B}-6-5,80-82\end{array}$ & $\begin{array}{l}92.31 \\
93.93 \\
95.31 \\
96.81 \\
98.31\end{array}$ & $\begin{array}{c}\mathrm{R} / \mathrm{F} \\
\mathrm{R} \\
\mathrm{F} \\
\mathrm{R} \\
\mathrm{R}\end{array}$ & $\begin{array}{l}\text { G } \\
G \\
G \\
G \\
G\end{array}$ & & & & \\
\hline $\begin{array}{l}482 \mathrm{~B}-6-6,80-82 \\
482 \mathrm{~B}-6, \mathrm{CC}(18-20) \\
482 \mathrm{~B}-7-1,80-82 \\
482 \mathrm{~B}-7-2,80-82 \\
482 \mathrm{~B}-7, \mathrm{CC}(3-5) \\
\end{array}$ & $\begin{array}{r}99.80 \\
100.70 \\
101.81 \\
103.31 \\
103.60 \\
\end{array}$ & $\begin{array}{l}\mathrm{R} \\
\mathrm{C} \\
\mathrm{R} \\
\mathrm{B} \\
\mathrm{B} \\
\end{array}$ & $\begin{array}{l}G \\
G \\
G\end{array}$ & & & & \\
\hline $\begin{array}{l}482 \mathrm{~B}-8-1,80-82 \\
482 \mathrm{D}-5, \mathrm{CC}(24-26) \\
482 \mathrm{C}-6-1,70-72 \\
482 \mathrm{~B}-8-2,80-82 \\
482 \mathrm{C}-6-2,70-72\end{array}$ & $\begin{array}{l}111.31 \\
111.70 \\
111.71 \\
112.80 \\
113.21\end{array}$ & $\begin{array}{l}\mathrm{B} \\
\mathrm{B} \\
\mathrm{B} \\
\mathrm{B} \\
\mathrm{B} \\
\end{array}$ & & & & & \\
\hline $\begin{array}{l}482 \mathrm{~B}-8-3,35-37 \\
482 \mathrm{~B}-8-3,56-58 \\
482 \mathrm{~B}-8-3,80-82 \\
482 \mathrm{~F}-2-1,82-84 \\
482 \mathrm{C}-6-3,70-72\end{array}$ & $\begin{array}{l}113.86 \\
114.07 \\
114.31 \\
114.33 \\
114.71 \\
\end{array}$ & $\begin{array}{l}\mathrm{B} \\
\mathrm{B} \\
\mathrm{B} \\
\mathrm{F} \\
\mathrm{B} \\
\end{array}$ & G & & & & \\
\hline $\begin{array}{l}482 \mathrm{~B}-8-4,80-82 \\
482 \mathrm{C}-6-4,70-72 \\
482 \mathrm{~B}-8-5,63-65 \\
482 \mathrm{~B}-8, \mathrm{CC}(3-5) \\
482 \mathrm{~F}-2, \mathrm{CC}(33-35)\end{array}$ & $\begin{array}{l}115.81 \\
116.71 \\
117.10 \\
117.38 \\
117.46\end{array}$ & $\begin{array}{l}\text { B } \\
\text { B } \\
\text { B } \\
\text { B } \\
\text { A }\end{array}$ & G & & & & \\
\hline $\begin{array}{l}482 \mathrm{C}-6-5,70-72 \\
482 \mathrm{C}-6-6,13-15 \\
482 \mathrm{C}-6-6,24-26 \\
482 \mathrm{~B}-9-1,80-82 \\
482 \mathrm{~B}-9-2,80-82\end{array}$ & $\begin{array}{l}117.71 \\
118.64 \\
118.75 \\
120.81 \\
122.31\end{array}$ & $\begin{array}{l}\text { B } \\
\text { B } \\
\text { B } \\
\text { B } \\
\text { B }\end{array}$ & & & & & \\
\hline $\begin{array}{l}482 \mathrm{~B}-9-3,80-82 \\
482 \mathrm{~F}-3-2,0-1 \\
482 \mathrm{~B}-9-4,85-87 \\
482 \mathrm{~B}-9-5,80-82 \\
482 \mathrm{~B}-9-6,85-87\end{array}$ & $\begin{array}{l}123.81 \\
124.50 \\
125.36 \\
126.81 \\
128.36 \\
\end{array}$ & $\begin{array}{l}\text { B } \\
\text { B } \\
\text { A } \\
\text { B } \\
\text { B } \\
\end{array}$ & G & & & & \\
\hline $\begin{array}{l}482 \mathrm{~B}-9, \mathrm{CC}(20-22) \\
482 \mathrm{~B}-10-1,80-82 \\
482 \mathrm{C}-8-1,30-32 \\
482 \mathrm{~B}-10-2,80-82 \\
482 \mathrm{C}-9-1,12-16\end{array}$ & $\begin{array}{l}128.96 \\
130.31 \\
130.31 \\
131.81 \\
132.14 \\
\end{array}$ & $\begin{array}{l}\mathrm{B} \\
\mathrm{R} \\
\mathrm{B} \\
\mathrm{F} \\
\mathrm{B} \\
\end{array}$ & $\begin{array}{l}\text { G } \\
\text { G }\end{array}$ & & & & \\
\hline $\begin{array}{l}\text { 482B-10-3, 80-82 } \\
\text { 482B-10-4, 80-82 } \\
\text { 482B-10-5, 80-82 } \\
\text { 482B-10-6, 80-82 } \\
\text { 482B-19-1, 49-51 }\end{array}$ & $\begin{array}{l}133.31 \\
134.81 \\
136.31 \\
137.81 \\
193.50 \\
\end{array}$ & $\begin{array}{l}\mathrm{R} \\
\mathrm{R} \\
\mathrm{B} \\
\mathrm{R} \\
\mathrm{B} \\
\end{array}$ & $\begin{array}{l}\text { G } \\
\text { G } \\
\text { G }\end{array}$ & & & & \\
\hline $482 \mathrm{~B}-24-1,21-24$ & 224.72 & R & G & & & & \\
\hline
\end{tabular}

Note: Abundances are indicated as: A (abundant), C (common), F (few), R (rare), and $\mathrm{B}$ (barren); blank space $=$ species searched for but not found. Preservation is indicated as $\mathrm{G}$ (good), M (moderate), and P (poor).

level occurs between 1 and 10 meters above the top of calcareous nannofossil Zone NN19, as determined by J. Hattner aboard the Glomar Challenger (this volume). The top of this zone (Pseudoemiliania lacunosa) has recently been dated at $0.44 \mathrm{Ma}$ by Gartner (1977). Johnson and Knoll (1975) claim that the highest occurrence of $A$. angelinum may be diachronous (Table 7), since it is significantly younger in two cores from the equatorial Pacific than in the North Pacific sediments 
Table 3. Radiolarians at Site $483 .^{\text {a }}$

\begin{tabular}{|c|c|c|c|c|c|c|c|c|c|c|}
\hline \multirow[b]{2}{*}{$\begin{array}{c}\text { Sample } \\
\text { (interval in } \mathrm{cm} \text { ) }\end{array}$} & \multirow[b]{2}{*}{$\begin{array}{l}\text { Depth below } \\
\text { Seafloor } \\
(m)\end{array}$} & \multirow[b]{2}{*}{ 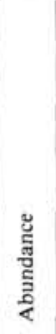 } & \multirow[b]{2}{*}{ 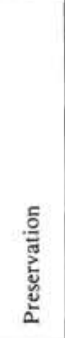 } & \multicolumn{7}{|c|}{ Taxa } \\
\hline & & & & 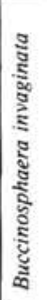 & 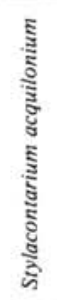 & 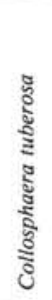 & 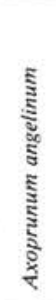 & 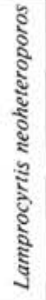 & 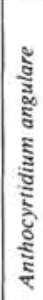 & 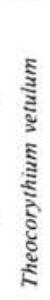 \\
\hline $\begin{array}{l}482-1, C C(13-15) \\
483-2, C C(5-7) \\
483-3-3,100-102 \\
483-3-4,23-25 \\
483-3-4,100-102\end{array}$ & $\begin{array}{r}0.40 \\
5.60 \\
14.51 \\
15.24 \\
16.01 \\
\end{array}$ & $\begin{array}{l}\mathrm{F} \\
\mathrm{A} \\
\mathrm{C} \\
\mathrm{C} \\
\mathrm{C}\end{array}$ & $\begin{array}{c}M / G \\
G \\
G \\
G \\
G\end{array}$ & & & & & & & \\
\hline $\begin{array}{l}483-4-1,100-102 \\
483-4, C C(0-3) \\
483-5-1,100-102 \\
483-5-2,100-102 \\
483-5-3,100-102\end{array}$ & $\begin{array}{l}21.01 \\
24.45 \\
30.51 \\
32.01 \\
33.51 \\
\end{array}$ & $\begin{array}{l}\mathrm{A} \\
\mathrm{A} \\
\mathrm{A} \\
\mathrm{A} \\
\mathrm{A}\end{array}$ & $\begin{array}{l}\mathrm{G} \\
\mathrm{G} \\
\mathrm{G} \\
\mathrm{G} \\
\mathrm{G}\end{array}$ & & & & $\mathrm{F}$ & & & \\
\hline $\begin{array}{l}483-5-4,100-102 \\
483-5, C \mathrm{C}(5-7) \\
483-6-2,28-31 \\
483-6, C \mathrm{CC}(16-18) \\
483-7, \mathrm{CC}(5-7)\end{array}$ & $\begin{array}{l}35.01 \\
37.10 \\
40.80 \\
43.60 \\
55.61 \\
\end{array}$ & $\begin{array}{l}\mathrm{A} \\
\mathrm{A} \\
\mathrm{A} \\
\mathrm{A} \\
\mathrm{C}\end{array}$ & $\begin{array}{c}G \\
G \\
G \\
G \\
M / G\end{array}$ & & & $\begin{array}{l}\mathrm{R} \\
\mathrm{R}\end{array}$ & $\begin{array}{c}\mathrm{R} \\
\mathrm{F} / \mathrm{C} \\
\mathrm{F} \\
\mathrm{C} \\
\mathrm{C}\end{array}$ & & & \\
\hline $\begin{array}{l}483-8-1,70-72 \\
483-8-2,70-72 \\
483-8-3,70-72 \\
483-8-4,70-72 \\
483-8, C C(11-13)\end{array}$ & $\begin{array}{l}58.71 \\
60.21 \\
61.71 \\
63.21 \\
63.65 \\
\end{array}$ & $\begin{array}{c}\mathrm{R} \\
\mathrm{F} / \mathrm{C} \\
\mathrm{R} \\
\mathrm{F} \\
\mathrm{A} \\
\end{array}$ & $\begin{array}{l}G \\
G \\
G \\
G \\
G\end{array}$ & & & & $\begin{array}{l}\mathrm{R} \\
\mathrm{R} \\
\mathrm{R} \\
\mathrm{C} \\
\mathrm{C} \\
\end{array}$ & $\mathbf{R}$ & & R \\
\hline $\begin{array}{l}483-9-1,74-76 \\
483-9, C C(10-12) \\
483-10, C C(19-21) \\
483 C-2-1,80-82 \\
483 C-2-3,80-82 \\
\end{array}$ & $\begin{array}{l}68.25 \\
77.14 \\
79.71 \\
86.81 \\
89.81 \\
\end{array}$ & $\begin{array}{l}\mathrm{A} \\
\mathrm{C} \\
\mathrm{R} \\
\mathrm{R} \\
\mathrm{R} \\
\end{array}$ & $\begin{array}{l}G \\
G \\
G \\
G \\
G\end{array}$ & & & & $\begin{array}{l}\mathrm{C} \\
\mathrm{A} \\
\mathrm{R} \\
\mathrm{R} \\
\end{array}$ & R & $\begin{array}{c}\mathrm{R} \\
\mathrm{cf} .\end{array}$ & \\
\hline $\begin{array}{l}483-11, C C 16-18 \\
483 C-2-6,80-82 \\
483 C-3-1,80-82 \\
483 C-3, C C(5-6) \\
483 B-2-1,70-72\end{array}$ & $\begin{array}{r}93.70 \\
94.31 \\
96.31 \\
98.60 \\
101.70 \\
\end{array}$ & $\begin{array}{l}\mathrm{B} \\
\mathrm{B} \\
\mathrm{B} \\
\mathrm{B} \\
\mathrm{B}\end{array}$ & & & & & & & & \\
\hline $\begin{array}{l}483-12, C C(4-6) \\
483 B-2-4,70-72 \\
483 B-2-6,81-83 \\
483-13-3,85-87 \\
483-17-1,10-13 \\
\end{array}$ & $\begin{array}{l}105.64 \\
106.21 \\
109.32 \\
109.36 \\
142.12 \\
\end{array}$ & $\begin{array}{l}\mathrm{B} \\
\mathrm{B} \\
\mathrm{B} \\
\mathrm{R} \\
\mathrm{B}\end{array}$ & G & & & & & & & \\
\hline $\begin{array}{l}483-18-2,0-2 \\
483-18-2,46-48 \\
483-18-2,130-132 \\
483-18-3,99-101 \\
483-18-3,124-126\end{array}$ & $\begin{array}{l}152.51 \\
152.97 \\
153.81 \\
155.00 \\
155.25 \\
\end{array}$ & $\begin{array}{l}\mathrm{B} \\
\mathrm{B} \\
\mathrm{B} \\
\mathrm{B} \\
\mathrm{B} \\
\end{array}$ & & & & & & & & \\
\hline $\begin{array}{l}483-18-4,70-72 \\
483-26-1,42-51 \\
483 \mathrm{~B}-20-2,71-95 \\
483 \mathrm{~B}-20-2,120-130 \\
483 \mathrm{~B}-25-2,6-8\end{array}$ & $\begin{array}{l}156.21 \\
200.47 \\
210.83 \\
211.25 \\
232.57\end{array}$ & $\begin{array}{l}\mathrm{B} \\
\mathrm{B} \\
\mathrm{B} \\
\mathrm{R} \\
\mathrm{B}\end{array}$ & G & & & & & & & \\
\hline
\end{tabular}

a See note, Table 2, for explanation of symbols.

studied by Hays (1970). In rather weak support of Johnson and Knoll's claim of diachroneity, single specimens of Collosphaera tuberosa occur 3.6 and 7.3 meters below the highest occurrence of $A$. angelinum in Hole 483 (Table 3). According to them, the first occurrence of $C$. tuberosa is dated at $0.37 \pm 0.01 \mathrm{Ma}$ in the two cores they studied.

\section{Biostratigraphy of Leg 65 Sites}

Tables 2-5 and Figure 4 summarize data from the Leg 65 holes for each site and are arranged according to subbottom depth. Marker species that I searched for are given in the tables. The datum for comparing all four sites in Figure 4 is the highest occurrence of Axoprunum angelinum at Sites 483, 484, and 485 . All of the sedi-
Table 4. Radiolarians at Site 484 a

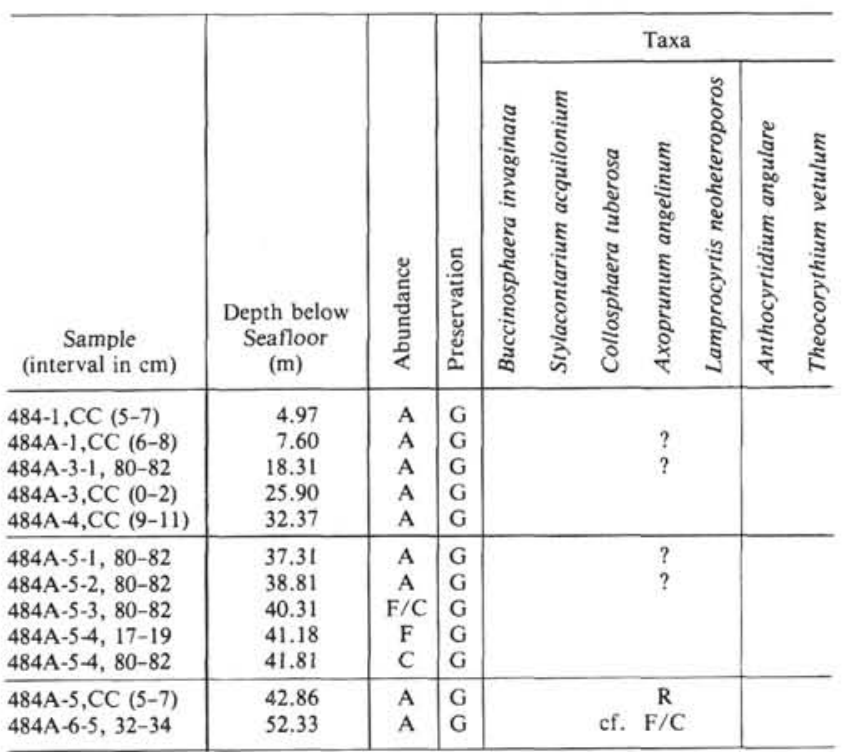

a See note, Table 2, for explanation of symbols.

mentary section recovered at Site 482 was deposited above this datum.

One feature apparent in Figure 4 is that in the few meters or tens of meters of sediment immediately overlying the basement and in the sediments interbedded with the basalt layers at Sites 482,483 , and 485 , radiolarians are generally absent or, if present, are rare but well preserved. Therefore, it does not appear that submarine volcanism had a direct effect on the preservation of radiolarian skeletons in these sediments.

In order to determine whether there is any pattern in the change in radiolarian abundance from rare or barren in the lower part to common and abundant in the upper part of the Pleistocene section in the region of the mouth of the Gulf of California, I have combined the data from Leg 65 with radiolarian data from Legs 63 and 64 in Table 8.

The faunal increase clearly occurred much earlier at sites northwest of the axis of the East Pacific Rise than at sites southeast of the axis (Fig. 1) and must have resulted, at least in part, from enhanced biological productivity in overlying waters. Perhaps upwelling or the influence of the California Current System was felt earlier at the northwestern sites during the opening of the Gulf than at the southeastern ones. Alternatively, more nearly oceanic conditions, but not necessarily upwelling, with concomitant increased contribution of radiolarian skeletons to the bottom sediments, would have prevailed earlier at the northwestern sites, which are farther from the Mexican mainland than the southeastern sites and were, therefore, less influenced by terrigenous sedimentation.

\section{Site $\mathbf{4 8 2}$}

None of the Quaternary-age radiolarian marker species (Table 7) was found in any of the samples from the drill holes at Site 482 (Table 2). All of the sediment at 
Table 5. Radiolarians at Site $485 .^{\mathrm{a}}$

\begin{tabular}{|c|c|c|c|c|c|c|}
\hline \multirow[b]{2}{*}{$\begin{array}{c}\text { Sample } \\
\text { (interval in } \mathrm{cm} \text { ) }\end{array}$} & \multirow[b]{2}{*}{$\begin{array}{l}\text { Depth below } \\
\text { Seafloor } \\
\text { (m) }\end{array}$} & \multirow[b]{2}{*}{ 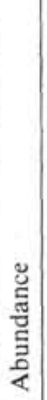 } & \multirow[b]{2}{*}{ 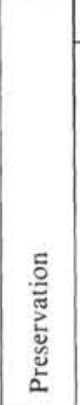 } & \multicolumn{3}{|c|}{ Taxa } \\
\hline & & & & 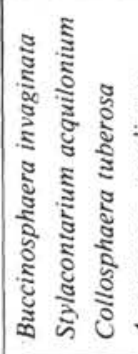 & 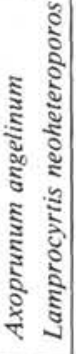 & 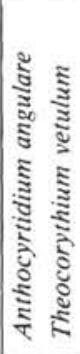 \\
\hline $\begin{array}{l}485-1-2,80-82 \\
485-2, C C(7-9) \\
485-3, C C(7-9) \\
485-4, C C(13-15) \\
485-5-1,80-82\end{array}$ & $\begin{array}{r}2.31 \\
7.83 \\
22.07 \\
25.84 \\
32.31\end{array}$ & $\begin{array}{l}\mathrm{C} \\
\mathrm{C} \\
\mathrm{R} \\
\mathrm{A} \\
\mathrm{R}\end{array}$ & $\begin{array}{c}M \\
M / G \\
M / G \\
G \\
G\end{array}$ & & $?$ & \\
\hline $\begin{array}{l}485-5-2,80-82 \\
485-5-3,80-82 \\
485-5-4,80-82 \\
485-5, C C(5-7) \\
485-6, C C(6-8)\end{array}$ & $\begin{array}{l}33.81 \\
35.31 \\
36.81 \\
37.77 \\
50.58\end{array}$ & $\begin{array}{l}\mathrm{R} \\
\mathrm{R} \\
\mathrm{R} \\
\mathrm{C} \\
\mathrm{C}\end{array}$ & $\begin{array}{l}\mathrm{G} \\
\mathrm{G} \\
\mathrm{G} \\
\mathrm{G} \\
\mathrm{G}\end{array}$ & & $\begin{array}{l}R \\
R \\
F \\
F\end{array}$ & \\
\hline $\begin{array}{l}485 \mathrm{~A}-1, \mathrm{CC}(11-13) \\
485 \mathrm{~A}-2, \mathrm{CC}(19-21) \\
485 \mathrm{~A}-3, \mathrm{CC}(2-4) \\
485 \mathrm{~A}-4, \mathrm{CC}(3-4) \\
485 \mathrm{~A}-5, \mathrm{CC}(7-9)\end{array}$ & $\begin{array}{l}60.10 \\
65.10 \\
73.22 \\
80.15 \\
93.70 \\
\end{array}$ & $\begin{array}{l}\mathrm{R} \\
\mathrm{F} \\
\mathrm{A} \\
\mathrm{R} \\
\mathrm{B}\end{array}$ & $\begin{array}{l}\text { G } \\
G \\
G \\
G\end{array}$ & & $\begin{array}{ll}\mathrm{R} & \\
\mathrm{C} & \mathrm{R}\end{array}$ & cf. \\
\hline $\begin{array}{l}485 \mathrm{~A}-6, \mathrm{CC}(17-19) \\
485 \mathrm{~A}-7, \mathrm{CC}(3-5) \\
485 \mathrm{~A}-8, \mathrm{CC}(5-7) \\
485 \mathrm{~A}-9, \mathrm{CC}(15-17) \\
485 \mathrm{~A}-10, \mathrm{CC}(9-11)\end{array}$ & $\begin{array}{l}102.30 \\
109.70 \\
120.24 \\
129.88 \\
139.60\end{array}$ & $\begin{array}{l}\mathrm{B} \\
\mathrm{R} \\
\mathrm{B} \\
\mathrm{B} \\
\mathrm{B}\end{array}$ & G & & & \\
\hline $\begin{array}{l}485 \mathrm{~A}-11-2,145-147 \\
485 \mathrm{~A}-19-2,10-12 \\
485 \mathrm{~A}-19-2,114-116 \\
485 \mathrm{~A}-20-2,13-15 \\
485 \mathrm{~A}-22, \mathrm{CC}(12-16)\end{array}$ & $\begin{array}{l}148.46 \\
189.61 \\
190.65 \\
194.14 \\
210.00\end{array}$ & $\begin{array}{l}\mathrm{B} \\
\mathrm{B} \\
\mathrm{R} \\
\mathrm{B} \\
\mathrm{B}\end{array}$ & G & & & \\
\hline $\begin{array}{l}485 \mathrm{~A}-26, \mathrm{CC} \\
485 \mathrm{~A}-27, \mathrm{CC}(15-17) \\
485 \mathrm{~A}-28, \mathrm{CC}(15-19) \\
485 \mathrm{~A}-34-1,9-11 \\
485 \mathrm{~A}-34-1,36-39\end{array}$ & $\begin{array}{l}227.70 \\
231.97 \\
235.80 \\
277.10 \\
277.38 \\
\end{array}$ & $\begin{array}{l}\mathrm{B} \\
\mathrm{B} \\
\mathrm{B} \\
\mathrm{R} \\
\mathrm{B}\end{array}$ & $\mathrm{M} / \mathrm{G}$ & & $\mathrm{R}$ & \\
\hline $\begin{array}{l}485 A-36-2,130-150 \\
485 A-37, C C(11-12) \\
485 A-38-1,40-50\end{array}$ & $\begin{array}{l}297.90 \\
306.00 \\
313.50\end{array}$ & $\begin{array}{l}\mathrm{B} \\
\mathrm{B} \\
\mathrm{B}\end{array}$ & & & & \\
\hline
\end{tabular}

a See note, Table 2, for explanation of symbols.

the site must have been deposited above the radiolarian datum level denoted by the highest occurrence of Axoprunum angelinum (0.41 Ma; Fig. 4). This interpretation is supported by the absence of evidence for the existence at Site 482 of calcareous nannofossil Zone NN19, which ended 0.44 Ma (Gartner, 1977).

Radiolarian skeletons are well preserved throughout the sedimentary section. As mentioned, above the faunal increase at about 102 meters sub-bottom, radiolarians are present in all samples. Fluctuations in abundance are partly related to reduction of their numbers through dilution by fine sand, silt, and foraminifers transported to the site by turbidity currents or some other mechanism. With few exceptions, only the more pelagic sediments have common to abundant radiolarians.

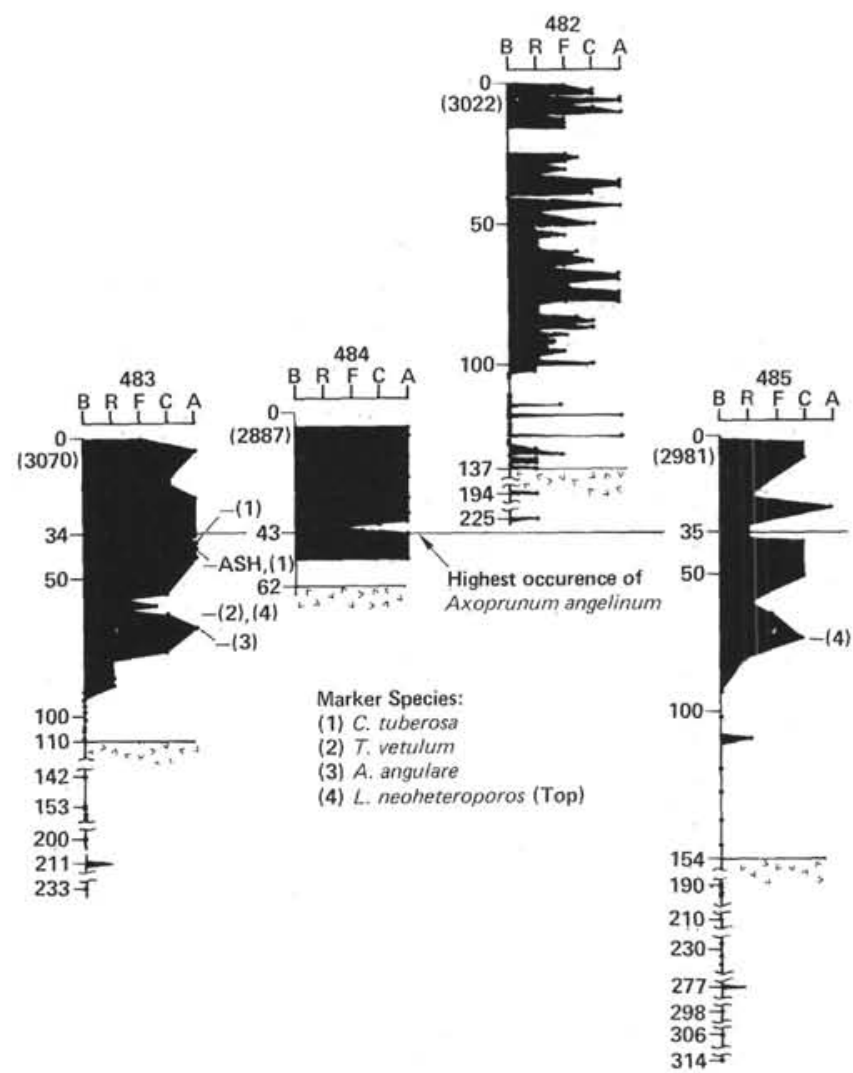

Figure 4. Leg 65 drill sites showing radiolarian occurrences and correlation using the highest occurrence of Axoprunum angelinum ( $=$ Stylatractus universus) as a datum. (Depth below mudline shown in meters on left of each column; water depth in parentheses. B, R, $\mathrm{F}, \mathrm{C}$, and $\mathrm{A}$ correspond to barren, rare, few, common, and abundant, respectively.)

\section{Site $\mathbf{4 8 3}$}

Above 80 meters sub-bottom, well-preserved radiolarians are generally common to abundant at Site 483 (Table 3). Below this depth and in the sediments interlayered with the basalts, they are rare or absent but still well preserved. In the lowest sample with common radiolarians, Sample $483-9, \mathrm{CC}(10-12 \mathrm{~cm})$, the dominant forms are thick-walled actinommids, including abundant Axoprunum angelinum, Actinomma spp., Xiphatractus spp., Druppatractus spp., and a few robust nasselline species, including Theocalyptra davisiana davisiana, Botryostrobus aquilonaris, Carpocanarium papillosum, Plectopyramis dodecomma, and Cornutella profunda. Although there is little indication of chemical attack, this concentration of robust skeletons may have resulted from the dissolution of less solutionresistant skeletons from an originally more diverse assemblage typical of the overlying sediments.

The highest occurrence of Axoprunum angelinum is in Sample 483-5-3, 100-102 cm (33.51 m sub-bottom). At this level, as well as at its highest occurrences at Sites 484 and 485 , I observed several specimens in which the two polar spines were reduced or absent (Plate 1, Figs. 3 
Table 6. Quaternary planktonic foraminifers at Site $483 .^{\mathrm{a}}$

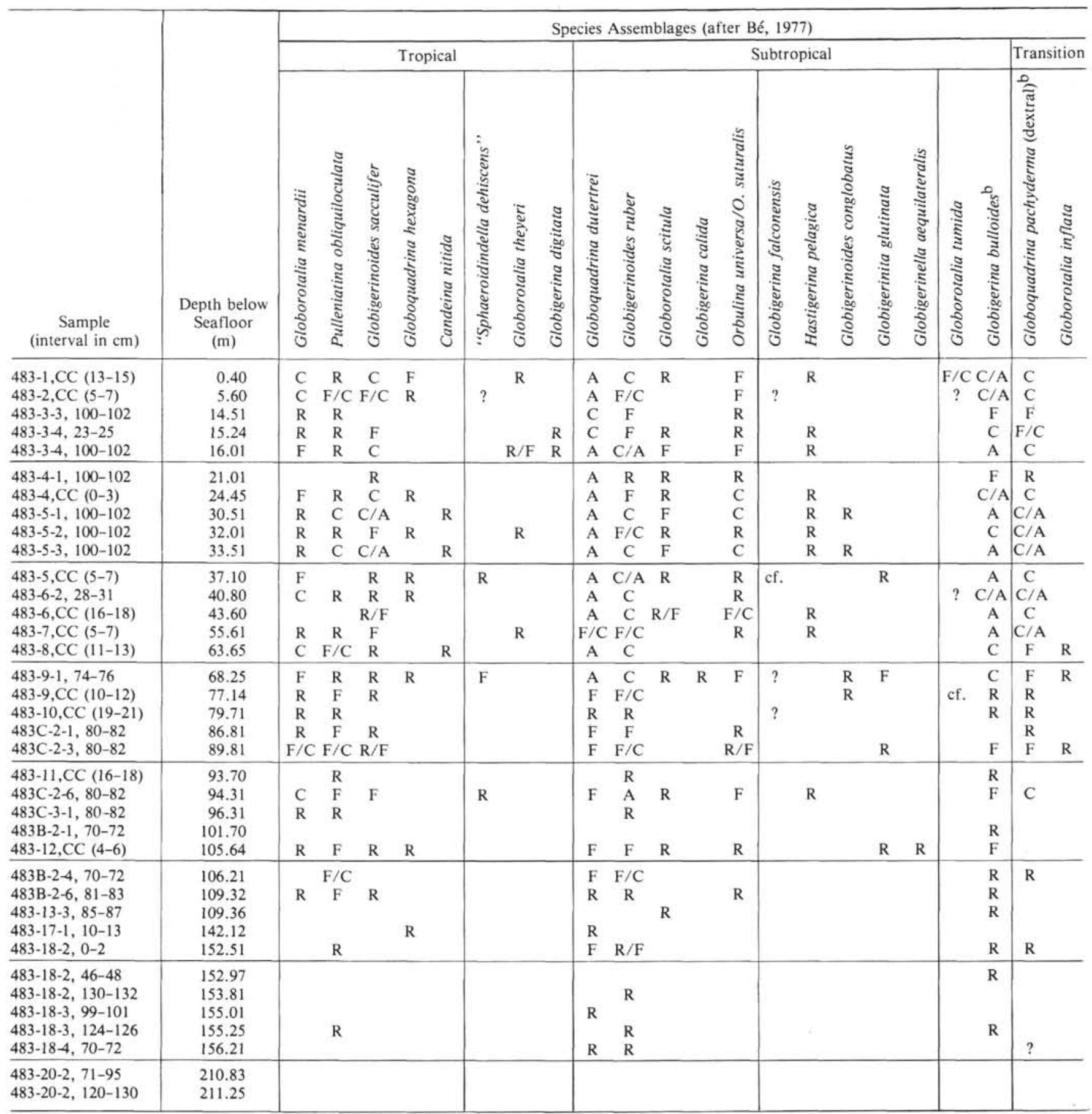

a See note, Table 2, for explanation of symbols.

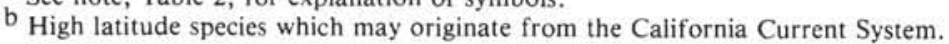

and 4). A. angelinum is present in every sample down to the section which is barren of radiolarians.

Other Quaternary marker species that I noted include: (1) single occurrences of Collosphaera tuberosa in Samples 483-5,CC (5-7 cm) (Plate 1, Figs. 5-6) and 483-6-2, 16-18 cm, the latter being a sample of a volcanic ash layer; (2) rare occurrences of Lamprocyrtis neoheteroporos in Samples 483-8,CC (11-13 cm) (Plate 3, Figs. 4, 6) and 483-9-1, 74-76 cm; (3) one specimen identified as Anthocyrtidium angulare in Sample 483-
9-1, 74-76 cm (Plate 3, Fig. 1), and (4) one specimen identified as Theocorythium vetulum in Sample 483$8, \mathrm{CC}(11-13 \mathrm{~cm})$ (Plate 3, Figs. 7-9). Because of the scarcity of these species, I did not assign any of the section at Site 483 to Nigrini's (1971) fourfold zonation of the Quaternary.

\section{Site 484}

Well-preserved radiolarians are abundant in nearly all of the samples examined from Site 484 (Table 4). The 
Table 7. Estimated ages of Quaternary radiolarian datum levels.

\begin{tabular}{|c|c|c|}
\hline Radiolarian Datum Level & $\begin{array}{l}\text { Estimated Age } \\
\text { (Ma) }\end{array}$ & References and Remarks \\
\hline $\begin{array}{l}\text { Transition: Collosphaera sp. A. } \\
\text { to Buccinosphaera invaginata }\end{array}$ & $0.21 \pm 0.02$ & Johnson and Knoll (1975) \\
\hline Top: Stylacontarium acquilonium & 0.31 & Hays (1970) \\
\hline (= Druppatractus acquilonius) & 0.4 & Kling (1973) \\
\hline Base: Collosphaera tuberosa & $0.37 \pm 0.01$ & Johnson and Knoll (1975) \\
\hline Top: Axoprunum angelinum & 0.32 & Johnson and Knoll (1975) \\
\hline (= Stylatractus universus) & $0.41 \pm 0.005$ & Hays and Shackleton (1976) \\
\hline \multirow[t]{3}{*}{ Top: Lamprocyrtis neoheteroporos } & 0.54 & $\begin{array}{l}\text { Johnson and Knoll's (1975) estimate based } \\
\text { on Kling's (1973) data for DSDP Site } 175\end{array}$ \\
\hline & 0.76 & $\begin{array}{l}\text { Johnson and Knoll's (1975) estimate based } \\
\text { on Kling's (1973) data for DSDP Site 173 }\end{array}$ \\
\hline & 1.03 & $\begin{array}{l}\text { Johnson and Knoll (1975) for tropical } \\
\text { Pacific }\end{array}$ \\
\hline Base: Collosphaera sp. A. & 0.61 & Johnson and Knoll (1975) \\
\hline Top: Anthocyrtidium angulare & 0.94 & Johnson and Knoll (1975) \\
\hline Top: Theocorythism vefulum & 0.94 & $\begin{array}{l}\text { By inference from Johnson and Knoll } \\
\text { (1975) because top same as A. angulare } \\
\text { (Nigrini, 1971) }\end{array}$ \\
\hline Top: Pterocanium prismatium & 1.70 & Johnson and Knoll (1975) \\
\hline
\end{tabular}

Table 8. Radiolarian abundance changes at DSDP sites in the mouth of the Gulf of California.

\begin{tabular}{|c|c|c|c|}
\hline Site & $\begin{array}{l}\text { Depth to } \\
\text { Basement } \\
\text { (m) }\end{array}$ & $\begin{array}{l}\text { Depth to Change } \\
\text { from Rare to Barren } \\
\text { Below to Common and } \\
\text { Abundant Above } \\
\text { (m) }\end{array}$ & $\begin{array}{c}\text { Date of Faunal Increase } \\
\text { Extrapolated from } \\
\text { Estimated Rates of } \\
\text { Sediment Accumulation } \\
\text { (Ma) }\end{array}$ \\
\hline \multicolumn{4}{|c|}{$\begin{array}{l}\text { Southeast of } \\
\text { East Pacific } \\
\text { Rise axis: }\end{array}$} \\
\hline $\begin{array}{l}473 \\
482 \\
485\end{array}$ & $\begin{array}{l}287 \\
137 \\
154\end{array}$ & $\begin{array}{c}29 \\
102 \\
73-81\end{array}$ & $\begin{array}{c}0.5 \\
<0.41 \\
0.48-0.58\end{array}$ \\
\hline \multicolumn{4}{|c|}{$\begin{array}{l}\text { Northwest of } \\
\text { East Pacific } \\
\text { Rise axis: }\end{array}$} \\
\hline $\begin{array}{l}474 \\
475 \\
476 \\
483\end{array}$ & $\begin{array}{l}563 \\
\mathrm{n} / \mathrm{a} \\
257 \\
110\end{array}$ & $\begin{array}{c}320 \\
45-53 \\
80 \\
80\end{array}$ & $\begin{array}{c}1.3 \\
0.85-1.3 \\
1.7 \\
1.2\end{array}$ \\
\hline
\end{tabular}

datum level represented by the highest occurrence of Axoprunum angelinum is at 42.86 meters sub-bottom (Sample 484A-5,CC [5-7 cm]). A few reworked specimens of this species were found above this depth in Samples $484 \mathrm{~A}-1, \mathrm{CC}(6-8 \mathrm{~cm}), 484 \mathrm{~A}-3-1,80-82 \mathrm{~cm}$, $484 \mathrm{~A}-5-1,80-82 \mathrm{~cm}$, and $484 \mathrm{~A}-5-2,80-82 \mathrm{~cm}$. A few reworked calcareous nannofossils from Zone NN19 also were found in some of the upper cores (J. Hattner, personal communication). I did not find other Quaternary radiolarian marker species at Site 484 .

\section{Site $\mathbf{4 8 5}$}

Radiolarians are common to abundant only in the upper 75-80 meters of the sediments at Site 485 (Table $5)$. Below this and in the sediments interbedded with the basalts, they are rare or absent. Wherever they are found, however, the radiolarians are well preserved.

Above 75-80 meters, the most abundant and diverse assemblages are from the more pelagic sediments characterized by the presence of in situ, lower bathyal to abyssal, benthic foraminifers. Radiolarians are generally few to rare in samples from fine-grained turbidites which lack the foraminiferal assemblages.

The highest occurrence of Axoprunum angelinum is in Sample 485-5-3, 80-82 cm (35.31 m sub-bottom), and it is present in almost all samples below this to a sub- bottom depth of about 80 meters (Table 5). The only other Quaternary marker species I observed is Lamprocyrtis neoheteroporos from Sample 485A-3,CC (2-4 $\mathrm{cm}$ ) (Plate 3, Fig. 5).

\section{SYSTEMATIC REFERENCE LIST}

The purpose of this list is to provide recent bibliographic references plus notes, where applicable, to the radiolarian taxa present in Holocene sediments from the Gulf of California (Benson, 1964, 1966) and in Quaternary sediments recovered during Leg 65 at the mouth of the Gulf. Original references are generally not given. Most of the generic assignments are those of recent authors; otherwise, the original authors' genera or those of Campbell (1954) were used.

Except for those taxa identified in the text as dominant members of the Pleistocene and Holocene assemblages, all of the taxa listed are generally rare. Nearly all ( $95 \%$ or more) of the taxa present are accounted for in the list.

\section{Acrobotrissa cribosa Popofsky}

(Plate 9, Fig. 5)

Acrobotrissa cribosa Popofsky, 1913, p. 322, text-fig. 29; Benson, 1966 , p. 342 , pl. 23 , fig. 15 , text-fig. 22; Casey, 1971, pl. 23.2, figs. 3-4.

\section{Acrobotrys sp. cf. A. disolenia Haeckel} (Plate 9, Figs. 6-7)

Cf. Acrobotrys disolenia Haeckel, 1887, p. 1114, pl. 96, fig. 10. Acrobotrys cf. disolenia Haeckel, Benson, 1966, p. 339, pl. 23. figs. 13-14, text-fig. 21.

\section{Acrosphaera murrayana Haeckel}

Choenicosphaera murrayana Haeckel, Benson, 1964, pl. 1, fig. 6; 1966 , p. 120 , pl. 2, fig. 3.

Polysolenia murrayana (Haeckel), Nigrini, 1968, p. 52, pl. 1, figs. $1 \mathrm{a}-\mathrm{b}$.

Remarks. According to Johnson and Nigrini (1980), the correct generic name for collosphaerids with irregularly scattered spines is Acrosphaera, not Polysolenia.

\section{Actinomma antarcticum (Haeckel)}

Diploplegma banzare Riedel, Benson, 1966, p. 134, pl. 2, fig. 14, pl. 3, figs. 2-3 (not fig. 1).

Actinomma antarcticum (Haeckel), Nigrini, 1967, p. 26, pl. 2, figs. 1a-d.

\section{Actinomma arcadophorum Haeckel}

Actinomma arcadophorum Haeckel, Nigrini, 1967, p. 29, pl. 2, fig. 3; 1970, p. 167, pl. 1, fig. 11.

\section{Actinomma leptodermum (Jörgensen)}

Actinomma sp., Benson, 1964, pl. 1, fig. 15; 1966, p. 164, pl. 5, fig. 6 (not fig. 5).

Echinomma leptodermum Jörgensen, Kling, 1977, p. 215, pl. 2, fig. 16.

Actinomma leptodermum (Jörgensen), Nigrini and Moore, 1979, p. S35, pl. 3, fig. 7.

\section{Actinomma medianum Nigrini}

Diploplegma banzare Riedel, Benson, 1966, p. 134, pl. 3, fig. 1. Actinomma medianum Nigrini, 1967, p. 27, pl. 2, figs. 2a-b; 1970, p. 167 , pl. 1 , fig. 10 .

\section{Actinomma sp.}

Actinomma sp., Benson, 1964, pl. 1, fig. 16; 1966, p. 164, pl. 5, fig. 5 .

\section{Actinosphaera cristata (Haeckel)(?)}

Cenosphaera cristata Haeckel?, Riedel, 1958, p. 223, pl. 1, figs. 1-2; Kling, 1977, p. 215, pl. 2, fig. 4.

Carposphaera acanthophora (Popofsky), Benson, 1964, pl. 1, fig. $1 ; 1966$, p. 127 , pl. 2, figs. 8-10. 
Actinosphaera acanthophora (Popofsky), Dumitrică, 1972, p. 832, pl. 20, figs. 1-2.

Haliomma erinaceum Haeckel, Renz, 1976, p. 101, pl. 2, figs. 4a-b. Remarks. Dumitrică $(1972$, p. 832) describes this species as having "a delicate primitive microsphere with large polygonal meshes which are connected to the cortical shell by a number of thread-like radial bars." Dumitrică (1972) assigned two such species to the genus $A c$ tinosphaera Hollande and Enjumet, and I have followed this practice for Cenosphaera cristata (Haeckel)(?).

\section{Amphiplecta cylindrocephala Dumitrică}

(Plate 8, Fig. 5)

Amphiplecta cf. acrostoma Haeckel, Benson, 1966, p. 464, pl. 32, figs. 1-2.

Amphiplecta cylindrocephala Dumitrică, 1972, p. 836, pl. 24, figs. 4-5.

\section{Amphirhopalum virchowii (Haeckel) \\ (Plate 2, Figs. 1-3)}

Amphirhopalum wirchowii (sic) (Haeckel), Dumitrică, 1972, p. 835, pl. 9, figs. 2, 4, pl. 11, fig. 6, pl. 21, figs. 2-13.

Remarks. Nigrini and Moore (1979, p. S76) note Dumitricǎ's minor spelling error. Both $A$. ypsilon and $A$, virchowii occur together in Leg 65 samples, although the latter is rare and is absent from Holocene sediments in the Gulf of California (Benson, 1966). The latest occurrence of $A$. virchowii in Leg 65 holes is in Sample $482 \mathrm{~A}-4, \mathrm{CC}$ ( $33.5 \mathrm{~m}$ subsea). All of the sediment at Site 482 is younger than the extinction datum for Axoprunum angelinum; therefore, $A$. virchowii ranges above this datum.

\section{Amphirhopalum ypsilon Haeckel}

(Plate 2, Figs. 4-7)

Amphicraspedum wyvilleanum Haeckel, Benson, 1964, pl. 1, figs. 2728 ; 1966 , p. 221 , pl. 11 , figs. 5-6.

Amphirhopalum ypsilon Haeckel, Nigrini, 1967, p. 35, pl. 3, figs. 3ad; 1970 , p. 168 , pl. 2, fig. 2; 1971, p. 447 , pl. 34.1, figs. 7a-c.

Remarks. Although I did not make counts of specimens, tests found lower in the Quaternary sections at Leg 65 sites generally have fewer chambers (three or four) on the forked arm before bifurcation (Plate 2, Figs. 4-6) than those found higher in the section (Plate 2, Fig. 7), a trend first noted by Nigrini (1971).

\section{Amphisphaera cristata Carnevale \\ (Plate 4, Fig. 5)}

Amphisphaera cf. uranus Haeckel, Benson, 1964, pl. 1, fig. 7; 1966, p. 136, pl. 3, figs. 4-5.

Amphisphaera cristata Carnevale, Dumitrică, 1972, p. 833, pl. 20, fig. 10.

\section{Amphitholus acanthometra Haeckel}

Amphitholus acanthometra Haeckel, 1887, p. 667; Benson, 1964, pl. 1, fig. 60 ; 1966 , p. 258 , pl. 17 , figs. 4-7.

\section{Anomalacantha dentata (Mast)}

Anomalacantha dentata (Mast), Benson, 1966, p. 170, pl. 5, figs. 10-11.

Heteracantha dentata Mast, Nigrini, 1970, p. 167, pl. 1, fig. 9.

\section{Anthocyrtidium angulare Nigrini}

(Plate 3, Fig. 1)

Anthocyrtidium angulare Nigrini, 1971, p. 445, pl. 34.1, figs. 3a-b; Dinkelman, 1973, p. 788, pl. 10, fig. 5; Johnson and Knoll, 1975, p. 107 , pl. 1, fig. 3 .

Remarks. The specimen illustrated in Plate 3, Figure 1 is the only one found in Leg 65 samples that has the dimensions and true birettashaped thorax described for this species by Nigrini (1971). It differs from Nigrini's (1971, pl. 34.1, figs. 3a-b) illustration of the species in the larger size of the thoracic pores, but Nigrini does not mention pore size as an identifying characteristic. Several somewhat larger specimens, having a cylindrical to slightly in-turned thorax beneath a sharp change in contour and herein designated Anthocyrtidium sp. cf. A. angulare (Plate 3, Figs. 2-3), were found at about the same level (Sam- ple 483-9-1, 74-76 cm) as A. angulare or lower. These specimens may be variant forms of Anthocyrtidium ophirense.

\section{Anthocyrtidium ophirense (Ehrenberg)}

Anthocyrtidium cineraria Haeckel, Benson, 1964, pl. 2, figs. 28-29; 1966 , p. 472 , pl. 32 , figs. 6-9.

Anthocyrtidium ophirense (Ehrenberg), Nigrini, 1967, p. 56, pl. 6, fig. 3; 1970, p. 171, pl. 4, fig. 7; Molina-Cruz, 1977, p. 337, pl. 6, fig. 10.

\section{Anthocyrtidium zanguebaricum (Ehrenberg)}

Anthocyrtium oxycephalis (Haeckel), Benson, 1964, pl. 2, fig. 27; 1966, p. 468 , pl. 32 , figs. $3-5$.

Anthocyrtidium zanguebaricum (Ehrenberg), Nigrini, 1967, p. 58, pl. 6, fig. 4; Molina-Cruz, 1977, p. 337, pl. 6, fig. 8.

\section{Arachnocorys umbellifera Haeckel}

(Plate 8, Fig. 6)

Arachnocorys umbellifera Haeckel, 1861, p. 837; 1862, p. 305, pl. 6, fig. 12; Benson, 1966, p. 375, pl. 24, figs. 20-21.

\section{Axoprunum angelinum (Campbell and Clark)}

(Plate 1, Figs. 1-4)

Stylatractus universus Hays, 1970, p. 215, pl. 1, figs. 1-2; Kling, 1971, p. 1086, pl. 1, fig. 7; Dinkelman, 1973, p. 765, pl. 10, figs. 6-7.

Axoprunum angelinum (Campbell and Clark), Kling, 1973, p. 634, pl. 1, figs. 13-16, pl. 6, figs. 14-18; Johnson and Knoll, 1975, p. 107, pl. 1, fig. 5 .

Remarks. The highest occurrence of this species at Sites 483, 484, and 485 represents the only reliable radiolarian datum determined for Leg 65 drill holes. Many tests of this species at its highest occurrence in Leg 65 samples are characterized by the reduction (Plate 1, Fig. 3) or absence (Plate 1, Fig. 4) of the two polar spines.

\section{Botryocyrtis quinaria Ehrenberg}

Botryocyrtis cf. caput-serpentis Ehrenberg, Benson, 1966, p. 348, pl. 23, fig. 17, text-fig. 24 .

Botryocyrtis quinaria Ehrenberg, Renz, 1974, p. 789, pl. 18, fig. 19.

\section{Botryocyrtis scutum (Harting)}

Botryopyle sp., Benson, 1964, pl. 2, fig. 64; 1966, p. 345, pl. 23, fig. 16 , text-fig. 23.

Botryocyrtis scutum (Harting), Nigrini, 1967, p. 52, pl. 6, figs. 1a-c; Molina-Cruz, 1977, p. 338, pl. 6, fig. 14.

Botryocyrtis sp., Casey, 1971, pl. 23.3, fig. 1.

\section{Botryostrobus aquilonaris (Bailey)}

Siphocampium erucosum (Haeckel), Benson, 1964, pl. 2, fig. 63; 1966 , p. 527 , pl. 35 , figs. $18-20$.

Botryostrobus aquilonaris (Bailey), Nigrini, 1977, p. 246, pl. 1, fig. 1; Kling, 1979, p. 309, pl. 2, fig. 18.

\section{Botryostrobus auritus-australis (Ehrenberg) group}

Siphocampium cf. seriatus (Haeckel), Benson, 1964, pl. 2, fig. 62; 1966 , p. 521 , pl. 35 , figs. $12-13$.

Botryostrobus auritus-australis (Ehrenberg) group, Nigrini, 1977, p. 246, pl. 1, figs. 2-5; Kling, 1979, p. 309, pl. 2, fig. 17.

\section{Buccinosphaera invaginata Haeckel}

Buccinosphaera invaginata Haeckel, Nigrini, 1971, p. 445, pl. 34.1, fig. 2; Dinkelman, 1973, p. 764, pl. 10, fig. 3; Johnson and Knoll, 1975, p. 107, pl. 1, fig. 2; Knoll and Johnson, 1975, p. 63, pl. 1, figs. 3-6.

Remarks. Although I searched for this species in Leg 65 samples, I did not find it.

\section{Callimitra emmae Haeckel}

Callimitra emmae Haeckel, 1887, p. 1218, pl. 63, figs. 3-4; Benson, 1966 , p. 390 , pl. 25 , fig. 12 .

Callimitra sp., Renz, 1974, p. 789 , pl. 18, fig. $5 ; 1976$, p. 162, pl. 7 , fig. 1. 


\section{Calocyclas monumentum Haeckel}

Clathrocyclas(?) sp., Benson, 1966, p. 457, pl. 31, figs. 2-3.

Calocyclas monumentum Haeckel, Renz, 1974, p. 789, pl. 16, fig. 25; 1976, p. 128, pl. 5, fig. 1; Riedel and Sanfilippo, 1977, pl. 23, fig. 2 .

\section{Carpocanarium papillosum (Ehrenberg) group}

Eucyrtidium papillosum Ehrenberg, 1872a, p. 310, 1872b, pl. 7, fig. 10.

Dictyocephalus mediterraneus Haeckel, 1887, p. 1307, pl 62, fig. 2; Benson, 1964, pl. 2, fig. 33; 1966, p. 439, pl. 29, fig. 13.

Dictyocephalus papillosus (Ehrenberg), Riedel, 1958, p. 236, pl. 3, fig. 10, text-fig. 8 .

Dictyocryphalus papillosus (Ehrenberg), Nigrini, 1967, p. 63, pl. 6, fig. 6; Renz, 1976, p. 139, pl. 6, fig. 9; Molina-Cruz, 1977, p. 338, pl. 7 , fig. 13 .

Carpocanarium spp., Riedel and Sanfilippo, 1971, p. 1599, pl. 1I, figs. 18, 20, 22-25, pl. 2J, figs. 8, 9(?).

Carpocanarium papillosum (Ehrenberg), Renz, 1974, p. 789, pl. 17, fig. 21; Nigrini and Moore, 1979, p. N27, pl. 21, fig. 3.

\section{Carpocanistrum spp.}

Carpocanium petalospyris Haeckel, 1887, p. 1283, pl. 52, fig. 19; Benson, 1964 , pl. 2 , figs. $24-25$; 1966 , p. 434 , pl. 29 , figs. 9-10, fig. 25.

Carpocanium spp., Nigrini, 1970, p. 171, pl. 4, figs. 4-6.

Carpocanium sp., Casey, 1971, pl. 23.3, fig. 2; Molina-Cruz, 1977, p. 337, pl. 6, fig. 13 (not fig. 12).

Carpocanistrum spp., Riedel and Sanfilippo, 1971, p. 1596, pl. IG, figs. 1-6, 8(?), 9, 10,11(?), 12(?), 13; Dumitrică, 1972, p. 838, pl. 14 , fig. 4, pl. 15, figs. 11-12, pl. 24, figs. 1, 3, 6; Renz, 1974, p. 789 , pl. 17 , fig. $17 ; 1976$, p. 151 , pl. 6 , fig. 4.

Carpocaniidae, gen. et spp. indet., Kling, 1973, p. 638, pl. 5, figs. 15, 6(?).

Remarks. I agree with Nigrini's (1970, p. 171) discussion of this group.

\section{Carpocanistrum(?) sp.}

Unnamed, Benson, 1964, pl. 2, fig. 32

Carpocanistrum sp., Riedel and Sanfilippo, 1971, p. 1596, pl. 1G, fig. 7.

Remarks. As noted by Riedel and Sanfilippo (1971, p. 1596), more detailed morphological studies will be required to determine whether or not forms with a more distinct cephalis are included in Carpocanistrum.

\section{Carpocanistrum sp. A, Nigrini}

Carpocanium sp., Benson, 1964, pl. 2, fig. 23; 1966, p. 438, pl. 29 , figs. 11-12.

Carpocanium sp. A, Nigrini, 1968, p. 55, pl. 1, fig. 4; Molina-Cruz, 1977 , p. 337 , pl. 6, fig. 12 (not fig. 13).

Carpocanistrum sp. A, Nigrini and Moore, 1979, p. N25, pl. 21, fig. 2 .

\section{Cenosphaera coronata Haeckel}

Cenosphaera coronata Haeckel, Molina-Cruz, 1977, p. 333, pl. 1, fig. 4; Nigrini and Moore, 1979, p. S39, pl. 4, fig. 1.

\section{Cenosphaera(?) sp. aff. C. perforata Haeckel} (Plate 4, Fig. 4)

Aff. Cenosphaera perforata Haeckel, 1887, p. 66, pl. 26, fig. 10. Cenosphaera aff. perforata Haeckel, Benson, 1966, p. 125, pl. 2, figs. 6-7.

\section{Ceratospyris(?) sp. cf. C.(?) borealis Bailey}

Eucoronis(?) sp., Benson, 1966, p. 306, pl. 21, figs. 9-10. Acanthodesmiidae, gen. et sp. indet., Kling, 1973, pl. 2, figs. 8-13. Cf. Ceratospyris borealis Bailey, Nigrini and Moore, 1979, p. N9, pl. 19 , figs. 1a-d.

Remarks. The forms identified by Benson (1966) as Eucoronis(?) sp. bear a superficial resemblance to Ceratospyris borealis Bailey.

\section{Circodiscus microporus (Stöhr)}

Trematodiscus microporus Stöhr, 1880, p. 108, pl. 4, fig. 17 .

Ommatodiscus pantanellii Carnevale, 1908, p. 24, pl. 4, fig. 6; Benson, 1964 , pl. 1 , fig. $39 ; 1966$, p. 207 , pl. 9 , figs. $7-8$, pl. 10, fig. 1 , text-fig. 12.

Circodiscus microporus (Stöhr), Petrushevskaya and Kozlova, 1972, p. 526, pl. 19, figs. 1-7.

(?)Xiphatractus sp. cf. X. circularis (Clark and Campbell), Kling, 1973 , p. 635 , pl. 7, figs. 15-17 (not figs. 11-14).

Porodiscus microporus (Stöhr), Renz, 1974, p. 794, pl. 15, fig. 16 1976, p. 109, pl. 3, fig. 15.

\section{Cladococcus cervicornis Haeckel (Plate 4, Fig. 1)}

Cladococcus cervicornis Haeckel, 1862, p. 370, pl. 14, figs. 4-6.

Elaphococcus cervicornis (Haeckel), Benson, 1966, p. 172, pl. 6, fig. 1.

(?)Cladococcus scoparius Haeckel, Renz, 1974, p. 789, pl. 13, fig. 17; 1976, p. 101, pl. 2, fig. 5.

\section{Cladococcus stalactites Haeckel} (Plate 4, Fig. 2)

Cladococcus stalactites Haeckel, 1887, p. 227, pl. 27, fig. 4; Benson, 1966 , p. 173 , pl. 6 , figs. $2-3$.

(?)Cladococcus abietinus Haeckel, Renz, 1974, p. 789, pl. 13, fig. 18.

\section{Cladoscenium(?) sp. ef. C.(?) tricolpum (Haeckel)}

Cf. Cladoscenium tricolpum Haeckel, Jörgensen, 1899, p. 78; 1905, p. 134, pl. 15 , figs. $71-73$.

Cladoscenium cf. tricolpum (Haeckel) Jörgensen, Benson, 1964, pl. 2, fig. $15 ; 1966$, p. 387 , pl. 25 , figs. $10-11$.

\section{Clathrocanium sp. ef. C. coronatum Popofsky}

Cf. Clathrocanium coronatum Popofsky, 1913, p. 342, pl. 33, fig. 1. Clathrocanium cf. coronatum Popofsky, Benson, 1966, p. 394, pl. 26, figs. 1-2.

(?)Clathrocanium ornatum Popofsky, 1913, p. 343, pl. 33, fig. 2; Casey, 1971, pl. 23.3, fig. 3.

Clathrocanium sp., Renz, 1974, p. 789, pl. 18, fig. 3; 1976, p. 163, pl 7, fig. 5.

\section{Clathrocircus stapediüs Haeckel}

(Plate 7, Figs. 5-7)

Clathrocircus stapedius Haeckel, 1887, p. 962, pl. 92, fig. 8; Benson, 1966 , p. 307 , pl. 21, figs. 11-13, pl. 22, fig. 1(?) (not fig. 2); Goll, 1972 , p. 963 , pl. 51 , fig. 3 .

(?)Triceraspyris damaecornis Haeckel, Nigrini, 1967, p. 46, pl. 5, fig. 5.

Remarks. Nigrini's (1967, pl. 5, fig. 5) illustration of Triceraspyris damaecornis resembles Benson's (1966, pl. 21, fig. 11) Clathrocircus stapedius more closely than it does Dendrospyris damaecornis (Benson, 1966, pl. 22, fig. 2).

\section{Clathrocorys murrayi Haeckel}

Clathrocorys murrayi Haeckel, 1887, p. 1219, pl. 64, fig. 8; Benson, 1966 , p. 391 , pl. 25 , figs. $13-15$.

Clathrocorys sp., Renz, 1974, p. 789, pl. 18, fig. 4; 1976, p. 163, pl. 7, fig. 4.

\section{Clathromitra pterophormis Haecke} (Plate 9, Fig.8)

Clathromitra pterophormis Haeckel, 1887, p. 1219, pl. 57, fig. 8; Benson, 1966 , p. 399 , pl. 26 , fig. 4 .

\section{Collosphaera(?) sp. (Plate 4, Fig. 3)}

Polysolenia? sp., Benson, 1966, p. 119, pl. 2, figs. 1-2.

\section{Collosphaera sp. A, Knoll and Johnson}

Collosphaera sp. A, Knoll and Johnson, 1975, p. 63, pl. 1, figs. 1-2, 7, pl. 2, figs. 4-6; Johnson and Knoll, 1975, p. 107, pl. 1, fig. 1. 
Remarks. Although 1 searched for this species in Leg 65 samples, I did not find it.

\section{Collosphaera tuberosa Haeckel}

(Plate 1, Fig. 5-6)

Collośphaera tuberosa Haeckel, Nigrini, 1970, p. 166, pl. 1, fig. 1; 1971, p. 445, pl. 34.1, fig. 1; Dinkelman, 1973, p. 763, pl. 10, figs. 1-2; Johnson and Knoll, 1975, p. 107, pl. 1, fig. 4; Knoll and Johnson, 1975 , p. 63 , pl. 2, figs. 1-3; Molina-Cruz, 1977, p. 332, pl. 2, fig. 6 .

Remarks. I found only one individual of this species in Sample $483-5, \mathrm{CC}, 5-7 \mathrm{~cm}$ and one in Sample 483-6-2, 28-31 cm.

\section{Cornutella profunda Ehrenberg}

Cornutella profunda Ehrenberg, Riedel, 1958, p. 232, pl. 3, figs. 1-2; Benson, 1964, pl. 2, fig. 30; 1966, p. 430, pl. 29, figs. 7-8; Nigrini, 1967 , p. 60 , pl. 6, figs. 5a-c; Casey, 1971, pl. 23.1, fig. 9; Kling, 1973 , p. 635 , pl. 3 , figs. $1-4$, pl. 9 , figs. $8-17$; Renz, 1974 , p. 790 , pl. 17 , figs. $24-25 ; 1976$, p. 149 , pl. 7 , fig. 11 ; Kling, 1977 , p. 215 , pl. 1, fig. 19; 1979, p. 309, pl. 1, fig. 21 .

\section{Cubotholus regularis Haeckel}

Cubotholus cf. octoceras Haeckel, 1887, p. 681; Benson, 1966, p. 260, pl. 17 , fig. 8 .

Cubotholus regularis Haeckel, Renz, 1976, p. 113, pl. 1, fig. 18.

\section{Cypassis irregularis Nigrini}

Spongoliva cf. ellipsoides Popofsky, Benson, 1966, p. 190, pl. 8, figs. 6-7.

Cypassis irregularis Nigrini, 1968, p. 53, pl. 1, figs. 2a-c; Kling, 1977, p. 215 , pl. 2 , fig. 5 .

\section{Dendrospyris damaecornis (Haeckel)}

Clathrocircus stapedius Haeckel, Benson, 1966, p. 307, pl. 22, fig. $1(?)$, fig. 2.

Dendrospyris damaecornis (Haeckel), Goll, 1972, p. 963, pl. 50, figs. $1-4$, pl, 51, figs. 1-2.

Remarks. Benson (1966, p. 308 ) observed a few tests identified as Clathrocircus stapedius that have a bilocular cephalis completely latticed except for the dorsal face. One of the illustrated specimens (Benson, 1966, pl. 22, fig. 2) resembles the closely related species (Goll, $1968,1972)$ Dendrospyris damaecornis as illustrated by Goll $(1972, \mathrm{pl}$. 51 , figs. 1-2). The other specimen (Benson, 1966, pl. 22, fig. 1) may belong to either species.

Dendrospyris sp. aff. D. binapteronis Goll

Patagospyris? sp., Benson, 1966, p. 326, pl. 22, fig. 22, pl. 23, figs. 1-2.

Dendrospyris sp. aff. D. binapteronis Goll, Renz, 1974, p. 790, pl. 19, fig. 11 .

\section{Dictyocoryne profunda Ehrenberg}

Hymeniastrum koellikeri Haeckel, Benson, 1964, pl. 1, fig. 32(?), fig. $34 ; 1966$, p. 225 , pl. 12 , figs. 5-6 (not fig. 4).

Dictyocoryne profunda Ehrenberg, Ling and Anikouchine, 1967, p. 1489, pl. 191, fig. 6, pl. 192, fig. 6; Molina-Cruz, 1977, p. 334, pl. 4, fig. 4; Nigrini and Moore, 1979, p. S87, pl. 12, fig. 1.

\section{Dictyocoryne sp. \\ (Plate 6, Fig. 2)}

Hymeniastrum koellikeri Haeckel, Benson, 1966, p. 225, pl. 12, fig. 4 (not figs. 5-6).

Dictyocoryne sp., Ling and Anikouchine, 1967, p. 1489, pl. 191, figs. 4-5, pl. 192, figs. 4-5

Remarks. Although Nigrini and Moore (1979, pp. S87-S89) tentatively place specimens with chalice-shaped arms, as figured by Benson (1966) and Ling and Anikouchine (1967), in synonymy with D. truncatum, I believe they may represent a separate species or subspecies and have, therefore, designated them as Dictyocoryne sp.

\section{Dictyocoryne truncatum (Ehrenberg)}

(Plate 6, Fig. 1)

Dictyocoryne cf. truncatum (Ehrenberg), Benson, 1964, pl. 1, fig. 47; 1966 , p. 235 , pl. 15 , fig. 1.

Dictyocoryne truncatum (Ehrenberg), Nigrini and Moore, p. S89 (partim.), pl. 12, fig. $2 \mathrm{a}$ (not $2 \mathrm{~b}$ ).

Remarks. In deference to Nigrini and Moore's (1979, pp. S89-S90) opinion that Euchitonia triangulum (Ehrenberg), as figured by them (op. cit., pl. 12, fig. 2b) and Ling and Anikouchine (1967, pls. 189 and 190 , figs. 8-9), may belong to Dictyocoryne truncatum, 1 prefer to keep it as a separate species.

\section{Dictyophimus crisiae Ehrenberg}

Pterocorys? sp., Benson, 1964, pl. 2, fig. 20; 1966, p. 412, pl. 28, figs. 4-6.

Dictyophimus crisiae Ehrenberg, Nigrini, 1967, p. 66, pl. 6, figs. 7a-b; Kling, 1973, p. 636 , pl. 4, figs. 11-15, pl. 10, figs. 18-20; Renz, 1974 , p. 791 , pl. 17 , fig. 2.

Pterocorys hirundo Haeckel, Casey, 1971, pl. 23.1, figs. 6-7; Ling, Stadum, and Welch, 1971, p. 715, pl. 2, figs. 8-10; Molina-Cruz, 1977 , p. 338 , pl. 8 , fig. 9.

\section{Dictyophimus infabricatus Nigrini}

Dictyophimus infabricatus Nigrini, 1968, p. 56, pl. 1, fig. 6, Kling, 1977 , p. 215 , pl. 2 , fig. 8 ; 1979 , p. 309 , pl. 1 , figs. $25-26$; not Molina-Cruz, 1977, pl. 8, fig. 1.

\section{Dictyophimus platycephalus Haeckel} (Plate 8, Fig. 7)

Dictyophimus platycephalus Haeckel, 1887, p. 1198, pl. 60, figs. 4-5; Benson, 1966 , p. 385 , pl. 25 , figs. $7-9$.

(?)Dictyophimus tetracanthus Popofsky, Renz, 1974, p. 791, pl. 18, fig. $11 ; 1976$, p. 157 , pl. 6, fig. 11.

\section{Dictyophimus sp. ef. D. tripus Haeckel}

(Plate 8, Fig. 4)

Cf. Dictyophimus tripus Haeckel, 1862, p. 306, pl. 6, fig. 1.

Dictyophimus cf. tripus Haeckel, Benson, 1966, p. 380, pl. 25, figs. $2-3$, text-fig. 8 C.

\section{Disolenia quadrata (Ehrenberg)}

Disolenia cf. variabilis (Haeckel), Benson, 1966, p. 123, pl. 2, fig. 5. Disolenia quadrata (Ehrenberg), Nigrini, 1967, p. 19, pl. 1, fig. 5.

\section{Doryconthidium sp. cf. D. hexactis (Vinassa de Regny)}

Cf. Dorylonchidium hexactis Vinassa de Regny, 1900, p. 230, pl. 1, fig. 12.

Doryconthidium? sp., Benson, 1966, p. 146, pl. 3, fig. 12.

Actinommid 3 gen. and sp. indet., Renz, 1974, p. 787, pl. 14, fig. 9.

\section{Druppatractus irregularis Popofsky}

Druppotractus (sic) irregularis Popofsky, 1912, p. 114, figs. 24-26. Druppatractus irregularis Popofsky, Benson, 1964, pl. 1, fig. 19; 1966, p. 180, pl. 7, figs. 7-11.

\section{Druppatractus variabilis Dumitrică}

Druppatractus cf. pyriformis (Bailey), Benson, 1964, pl. 1, fig. 20; 1966 , p. 177 , pl. 7, figs. 2-6.

Druppatractus variabilis Dumitrică, 1972, p. 833, pl. 6, fig. 4, pl. 20, figs. 6-7.

\section{Echinomma delicatulum (Dogiel)}

Actinomma cf. hystrix (Müller), Benson, 1966, p. 166, pl. 5, figs. 3-4. Echinomma delicatum (Dogiel), Ling et al., 1971, p. 710, pl. 1, fig. 4; Molina-Cruz, 1977, p. 333, pl. 1, fig. 5.

Echinomma delicatulum (Dogiel), Kling, 1977, p. 215, pl. 2, fig. 9. 


\section{Eucecryphalus cervus (Ehrenberg)}

Coracalyptra (sic) cervus (Ehrenberg), Benson, 1964, pl. 2, figs. 52$53 ; 1966$, p. 447 , pl. 30 , figs. 3-5.

Corocalyptra cervus (Ehrenberg), Renz, 1974, p. 790, pl. 16, fig. 22; 1976, p. 129, pl. 5 , fig. 2 .

?Eucecryphalis (sic) cervus (Ehrenberg), Kling, 1977, p. 215, pl. 1, fig. 21.

Eucecryphalus (?) sp. (Plate 9, Fig. 4)

Eucecryphalus sp., Benson, 1966, p. 450, pl. 30, figs. 6-7; Casey, 1971, pl. 23.2, figs. 14-15.

\section{Euchitonia elegans (Ehrenberg)}

Euchitonia elegans (Ehrenberg), Benson, 1964, pl. 1, fig. 31(?); 1966, p. 230, pl. 14, fig. 1, fig. 2(?); Nigrini, 1967, p. 39, pl. 4, figs. 2a-b; 1970 , p. 169, pl. 2, fig. 6; Ling and Anikouchine, 1967, p. 1486, pls. 189 and 190, figs. 3-4; Molina-Cruz, 1977, p. 334, pl. 2, fig. 8; Nigrini and Moore, 1979, p. S83, pl. 11, figs. 1a-b.

Remarks. Although Nigrini and Moore (1979, p. S83) indicate that Benson's (1966) description (including dimensions) of this species is not consistent with theirs, E. elegans, along with $E$. furcata, is present in the Gulf of California and in Leg 65 samples. Benson's (1966, pl. 14 , fig. 1) illustration of $E$. elegans is similar to other published illustrations of this species.

\section{Euchitonia furcata Ehrenberg}

Euchitonia furcata Ehrenberg, Ling and Anikouchine, 1967, p. 1484, pls. 189 and 190, figs. 1-2, 5-7; Nigrini, 1970, p. 169, pl. 2, fig. 5; Nigrini and Moore, 1979, p. S85, pl. 11, figs. 2a-b.

(?)Euchitonia elegans (Ehrenberg), Benson, 1964, pl. 1, fig. 31; 1966 (partim.), p. 230, pl. 14, fig. 2 (not fig. 1).

Euchitonia mülleri Haeckel, Nigrini, 1967, p. 37, pl. 4, figs. 1a-b. Remarks. Benson's $(1964,1966)$ illustration of a test identified as Euchitonia elegans, with arms slightly expanded distally, may instead represent $E$. furcata.

\section{Euchitonia sp.}

Euchitonia mülleri Haeckel, Benson, 1964, pl. 1, fig. 30; 1966, p. 232, pl. 14, figs. 3-4.

Remarks. This species(?) is distinguished by: its large size; its circular central structure consisting of five to seven concentric, latticed, discoidal shells; its two similar arms which do not bend toward one another; and, in fully developed tests, a patagium with thickened margins, convex outward between the arms, presenting the appearance of a shield. It is clearly not the same as $E$. mülleri $(=E$. furcata $)$ of Nigrini (1967); therefore, I have designated it as Euchitonia sp.

\section{Euchitonia sp. cf. E. furcata Ehrenberg}

Euchitonia cf. furcata Ehrenberg, Benson, 1964, pl. 1, figs. 29, 33; 1966 , p. 228 , pl. 13, figs. 4-5.

Remarks. This species differs from Euchitonia furcata in having shorter arms that are broader and thicker in relation to their length, in having a patagium that is of similar thickness throughout its extent, and in having a central structure consisting of three to five concentric, discoidal, latticed shells with somewhat irregular outlines.

\section{Euchitonia sp. cf. E. triangulum (Ehrenberg)}

(Plate 5, Figs. 4-5)

Euchitonia cf. echinata Haeckel, Benson, 1966, p. 226, pl. 12, fig. 7, pl. 13, figs. 1-3.

Euchitonia cf. E. triangulum (Ehrenberg), Ling and Anikouchine, 1967 , p. 1487 , pl. 189 , figs. $8-9$, pl. 190 , figs $8-9$.

Dictyocoryne truncatum (Ehrenberg), Nigrini and Moore, 1979, p. S89, pl. 12, fig. $2 b$ (not $2 a$ ).

Remarks. This species is characterized by Benson (1966, p. 227) as being distinctly bilateral and having a circular central region consisting of five to eight concentric discoidal shells and an internal arm structure consisting of distinct, equally spaced, latticed rings, traceable from arm to arm. These features clearly distinguish this species from Dictyocoryne truncatum.

\section{Eucyrtidium(?) anomalum (Haeckel)}

Lithocampe anomala Haeckel, 1860, p. 839.

Eucyrtidium anomalum Haeckel, 1862, p. 323, pl. 7, figs. 11-13; Benson, 1964, pl. 2, fig. 56; 1966, p. 496, pl. 34, figs. 4-5; Renz, 1974, p. 791 , pl. 16 , fig. $20 ; 1976$, p. 131 , pl. 5 , fig. 8 .

Stichopterygium anomalum (Haeckel), Dumitrică, 1972, p. 838, pl. 27, fig. 11.

Remarks. This species is placed provisionally in the genus Eucyrtidium, although I observed no vertical tube on the cephalis. This feature is characteristic of Eucyrtidium infundibulum, $E$. hexagonatum, and E.(?) hexastichum (Benson, 1966, p. 505, text-fig. 26). Further study of Eucyrtidium and Eucyrtidium-like species seems warranted because of Dumitrică's $(1972$, p. 838$)$ rationale for placing E.(?) anomalum in the genus Stichopterygium. See also the remarks under E.(?) hexastichum.

\section{Eucyrtidium calvertense Martin}

Eucyrtidium calvertense Martin, Hays, 1970, p. 213, pl. 1, fig. 6; Kling, 1971 , p. 1088 , pl. 1 , fig. 3 ; 1973 , p. 636 , pl. 4 , figs. 16 , $18-19$, pl. 11, figs. $1-5$.

\section{Eucyrtidium hexagonatum Haeckel}

Eusyringium siphonostoma Haeckel, Benson, 1964, pl. 2, figs 55, 5960 ; 1966 , p. 498 , pl. 34 , figs. 6-9.

Eucyrtidium hexagonatum Haeckel, Nigrini, 1967, p. 83, pl. 8, figs. 4a-b; 1970, p. 171, pl. 4, fig. 2; Casey, 1971, pl. 23.3, fig. 5; Molina-Cruz, 1977 , p. 336 , pl. 7, figs. 4-5.

Remarks. Nigrini $(1967$, p. 83$)$ did not mention the inconspicuous vertical tube on the cephalis, which is characteristic of this species as well as of Eucyrtidium infundibulum and E.(?) hexastichum in the Gulf of California (Benson, 1966, p. 505, text-fig. 26).

\section{Eucyrtidium(?) hexastichum (Haeckel) group (Plate 9, Figs. 9-11)}

Lithostrobus hexastichus Haeckel, 1887, p. 1470, pl. 80, fig. 15; Benson, 1966 , p. 506, pl. 34, figs. 13-16.

Stichopilium annulatum Popofsky, 1913, p. 403, pl. 37, figs. 2-3.

Eucyrtidium hexastichum (Haeckel), Renz, 1974, p. 792, pl. 16, fig. 6; 1976, p. 132, pl. 5, fig. 9.

Remarks. Members of this Eucyrtidium-like group are similar in shape to $E$. hexagonatum and $E$. infundibulum and possess the indistinct vertical tube. They differ by having pores aligned transversely, not longitudinally. Because these forms have a lateral cephalic vertical tube and pores arranged in transverse rows, they would qualify for inclusion in the subfamily Artostrobiinae Riedel of the family Artostrobiidae Riedel emend. Foreman (Nigrini, 1977, p. 243), except that they have only four instead of six collar pores.

In the Gulf of California two general forms of this group are present. One has larger pores arranged in three to five transverse rows per abdominal segment (Benson, 1966, pl. 34, figs. 13-14; this chapter, pl.9, fig. 10). The other has smaller pores arranged in eight to twelve rows per segment (Benson, 1966, pl. 34, figs. 15-16; this chapter, pl. 9 , figs. 9, 11). The latter, as well as tests with pores of intermediate size, are less abundant in the Gulf sediments than are the former.

\section{Eucyrtidium infundibulum (Haeckel)}

Lithomitra infundibulum Haeckel, 1887, p. 1487, pl. 79, fig. 5; Benson, 1964 , pl. 2 , figs. $57-58$; 1966, p. 502, pl. 34, figs. 10-12, text-fig. 26 .

Remarks. Because of its similarity in shape to Eucyrtidium hexagonatum and the presence of an inconspicuous vertical tube on the cephalis, this species is placed in the genus Eucyrtidium.

\section{Eucyrtidium matuyamai Hays}

Eucyrtidium matuyamai Hays, 1970, p. 213, pl. 1, figs. 7-9; Kling, 1971, p. 1088, pl. 1, fig. 4; 1973, p. 636, pl. 4, fig. 17.

Remarks. I did not find this species in the Leg 65 drill hole samples examined.

\section{Giraffospyris angulata (Haeckel)}

Eucoronis nephrospyris Haeckel, Benson, 1964, pl. 2, fig. 6; 1966, p. 304 , pl. 21, figs. 6-8. 
Giraffospyris angulata (Haeckel), Goll, 1969, p. 331, pl. 59, figs. 4, 6-7, 9; Renz, 1974, p. 792, pl. 19, fig. 10; 1976, p. 167, pl. 8, fig. 5; Molina-Cruz, 1977, p. 336, pl. 6, fig. 7.

\section{Heliodiscus asteriscus Haeckel}

Heliodiscus asteriscus Haeckel, Benson, 1964, pl. 1, fig. 26; 1966, p. 200, pl. 9, fig. 3 (not fig. 4); Nigrini, 1967, p. 32, pl. 3, figs. 1a-b; 1970 , p. 168 , pl. 2 , fig. 1.

\section{Heliodiscus echiniscus Haeckel}

Heliodiscus asteriscus Haeckel, Benson, 1966, p. 200, pl. 9, fig. 4 (not fig. 3).

Heliodiscus echiniscus Haeckel, Nigrini, 1967, p. 34, pl. 3, figs. 2a-b.

Heliosphaera radiata Popofsky

Heliosphaera radiata Popofsky, 1912, p. 98, fig. 10; Benson, 1964, pl. 1, fig. $14 ; 1966$, p. 160 , pl. 5 , figs. $1-2$.

\section{Helotholus histricosa Jörgensen group (Plate 8, Figs. 1-3)}

Helotholus histricosa Jörgensen, Benson, 1966, p. 459, pl. 31, figs. 4-8; Kling, 1977, p. 215, pl. 2, fig. 6.

Remarks. Specimens from the Gulf that were identified as Helotholus histricosa Jörgensen are of two general types: (1) tests with a partially hidden cephalis and a discernible but indistinct collar stricture (Benson, 1966, pl. 31, figs. 4-5; this chapter, Plate 8, Fig. 2), and (2) tests with a completely hidden cephalis consisting of a broadly rounded cap-like structure with relatively large pores (Benson, 1966, pl. 31, figs. 6-8; this chapter, Plate 8, Figs. 1, 3).

\section{Hexacontium enthacanthum Jörgensen}

Hexacontium entacanthum (sic) Jörgensen, Benson, 1964, pl. 1, fig. 12; 1966, p. 149, pl. 3, figs. 13-14, pl. 4, figs. 1-3; Kling, 1977, p. 215 , pl. 2 , fig. 15 .

(?)Hexalonche anaximandri Haeckel, Renz, 1976, p. 103, pl. 2, fig. 8.

Hexacontium encanthum (sic) (Jörgensen), Molina-Cruz, 1977, p. 333 , pl. 2, fig. 5 (not fig. 3?).

Hexacontium enthacanthum Jörgensen, Nigrini and Moore, 1979, p. S45, pl. 5, figs. 1a-b.

\section{Hexacontium heteracantha (Popofsky)}

Hexalonche heteracantha Popofsky, 1912, p. 88, text-fig. 3.

Hexacontium cf. heteracantha (Popofsky), Benson, 1964, pl. 1, fig. $10 ; 1966$, p. 156 , pl. 4 , figs. $6-7$.

Actinomma sp. aff. Hexacontium arachnoidale Hollande and Enjumet, Petrushevskaya and Kozlova, 1972, p. 515, pl. 9, figs. 4-7. Remarks. This species is nearly identical with Hexacontium sp. cf. $H$. heracliti, and the two may be conspecific. Because both species are undoubtedly cubosphaerids with a constant number of six mutually perpendicular radial beams extended as main spines, I do not agree with Bjørklund's (1977) placement of them in synonymy with Actinomma haysi, unless it can be demonstrated that $A$. haysi is basically a cubosphaerid but with a variable number of additional beams and spines.

\section{Hexacontium laevigatum Haeckel}

Hexacontium laevigatum Haeckel, Benson, 1964, pl. 1, figs. 9, 13; 1966 , p. 153 , pl. 4 , figs. $4-5$; Molina-Cruz, 1977 , p. 333 , pl. 2 , fig. 7.

\section{Hexacontium sp. cf. $\mathrm{H}$. heracliti (Haeckel)}

(Plate 4, Fig. 7)

Cf. Hexalonche heracliti Haeckel, 1887, p. 187, pl. 22, fig. 7.

Hexacontium cf. hericliti (sic) (Haeckel), Benson, 1966, p. 158, pl. 4, figs. 8-10.

Remarks. See those under Hexacontium heteracantha.

\section{Hexapyle dodecantha Haeckel group}

$$
\text { (Plate 6, Figs. 6-7) }
$$

Hexapyle dodecantha Haeckel, 1887, p. 569, pl. 48, fig. 16; Benson, 1964 , pl. 1 , fig. $41 ; 1966$, p. 275 , pl. 18 , figs. $14-16$, text-fig. 20 .
Discopyle? sp., Benson, 1966, p. 271, pl. 18, figs. 11-13, text-fig. 19. Hexapyle spp., Molina-Cruz, 1977, p. 335, pl. 2, figs. 9-10.

Remarks. Fully developed individuals with an outer ellipsoidal shell of smooth outline (Benson, 1966, pl. 18, figs. 12, 13, 16; this chapter, Plate 6, Fig. 6) may closely resemble fully developed individuals of Phorticium pylonium and Larcopyle butschlii.

\section{Hexastylus triaxonius Haeckel}

(Plate 4, Fig. 6)

Hexastylus triaxonius Haeckel, 1887, p. 175, pl. 21, fig. 2; Benson, 1966 , p. 139 , pl. 3 , figs. $6-7$.

\section{Hymeniastrum euclidis Haeckel}

Hymeniastrum euclidis (Haeckel) Popofsky, Benson, 1964, pl. 1, fig. 45 ; 1966 , p. 222 , pl. 12 , figs. $1-3$.

Hymeniastrum euclidis Haeckel, Nigrini, 1970, p. 168, pl. 2, fig. 4; Kling, 1977, p. 215, pl. 2, fig. 6.

\section{Lamprocyclas maritalis maritalis Haeckel}

Lamprocyclas maritalis Haeckel, Benson, 1964, pl. 2, figs. 41-42; 1966 , p. 475 , pl. 32 , fig. 12 , pl. 33 , fig. 1(?).

Lamprocyclas maritalis maritalis Haeckel, Nigrini, 1967, p. 74, pl. 7, fig. 5; 1970, p. 171, pl. 4, fig. 9; Molina-Cruz, 1977, p. 337, pl. 6, figs. 8-9.

Remarks. Lamprocyclas maritalis maritalis was distinguished from $L . m$. polypora on the basis of having ten or fewer pores on the half equator of the abdomen, a thicker abdominal wall with pores set in polygonal frames, generally smaller abdominal dimensions, and, typically, a well-developed hyaline peristome with numerous toothlike spines. Some specimens with very broad abdomens (Benson, 1966 , pl. 33, fig. 1) may belong to $L . m$, ventricosa (Nigrini, 1968). $L$. $m$. maritalis is the dominant member of this group in the samples examined.

\section{Lamprocyclas maritalis Haeckel polypora Nigrini}

Lamprocyclas maritalis Haeckel, Benson, 1966, p. 475, pl. 32, figs. 10-11, pl. 33, fig. 1(?).

Lamprocyclas maritalis Haeckel polypora Nigrini, 1967, p. 76, pl. 7, fig. 6; 1970, p. 171, pl. 4, fig. 8; Molina-Cruz, 1977, p. 337, pl. 6, fig. 6 .

\section{Lamprocyrtis(?) hannai (Campbell and Clark)}

Lamprocyrtis(?) hannai (Campbell and Clark), Kling, 1973, p. 638, pl. 5, figs. 12-14, pl. 12, figs. 10-14.

Lamprocyclas junonis Haeckel, Molina-Cruz, 1977, p. 337, pl. 7, fig. 10.

\section{Lamprocyrtis neoheteroporos Kling}

(Plate 3, Figs. 4-6)

Lamprocyrtis neoheteroporos Kling, 1973, p. 639, pl. 5, figs. 17-18, pl. 15, figs. 4-5; Johnson and Knoll, 1975, p. 109, pl. 1, fig. 9; Riedel and Sanfilippo, 1978, p. 69, pl. 5, fig. 10.

\section{Lamprocyrtis nigriniae (Caulet)}

Conarachnium sp., Benson, 1964, pl. 2, fig. 31; 1966, p. 479, pl. 33, figs. 2-3.

Conarachnium? sp., Nigrini, 1968, p. 56, pl. 1, fig. 5a, 5b(?).

Lamprocyrtis haysi Kling, 1973, p. 639, pl. 5, figs. 15-16, pl. 15, figs. 1-3; Molina-Cruz, 1977, p. 337, pl. 6, fig. 9.

Lamprocyrtis nigriniae (Caulet) $(=$ L. haysi, Kling, 1973), Kling, 1977, p. 217, pl. 1, fig. 17; Nigrini and Moore, 1979, p. N81, pl. 25, fig. 7.

\section{Lampromitra quadricuspis Haeckel}

(Plate 8, Fig. 8)

Lampromitra quadricuspis Haeckel, 1887, p. 1214, pl. 58, fig. 7; Benson, 1966, p. 455 , pl. 30 , fig. 11, pl. 31 , fig. 1 .

\section{Larcopyle butschlii Dreyer group}

Larcopyle butschlii Dreyer, 1889, p. 124, pl. 10, fig. 70; Benson, 1966, p. 280 , pl. 19 , figs. $3-5$. 
Larcopyle? sp., Benson, 1966, p. 279, pl. 19, figs. 1-2.

Larcopyle butschlii Dreyer, 1889(?), Kling, 1977, p. 217, pl. 1, fig. 11. Remarks. This group of ellipsoidal tests with regular outline is identified on the basis of its internal pylonid structure and the presence of a cluster of spines at one pole of the test.

\section{Larcospira quadrangula Haeckel}

Larcospira quadrangula Haeckel, Benson, 1966, p. 266, pl. 18, figs. 7-8; Nigrini, 1970, p. 169, pl. 2, fig. 9; Casey, 1971, pl. 23.3, fig. 8; Kling, 1977, p. 217, pl. 2, fig. 18; Molina-Cruz, 1977, p. 335, pl. 3 , fig. 3 .

\section{Lipmanella dictyoceras (Haeckel)}

Dictyoceras acanthicum Jörgensen, Benson, 1964, pl. 2, fig. 37; 1966, p. 417 , pl. 28 , figs. $8-10$.

(?)Lipmanella irregularis (Cleve), Dumitrică, 1972, p. 840, pl. 25, fig. 2 .

Lipmanella dicytoceras (Haeckel), Kling, 1973, p. 636, pl. 4, figs. 2426; 1977, p. 217, pl. 2, fig. 2.

Lithopilium sphaerocephalum Popofsky, Renz, 1974, p. 794, pl. 16, fig. $12 ; 1976$, p. 123 , pl. 4, fig. 8 .

\section{Lipmanella tribranchiata Dumitrică}

(Plate 9, Fig. 1)

Dictyoceras cf. pyramidale (Popofsky), Benson, 1966, p. 419, pl. 28, fig. 11.

Lipmanella tribranchiata Dumitrică, 1972, p. 840 , pl. 25, figs. 3-5.

\section{Liriospyris reticulata (Ehrenberg)}

Amphispyris toxarium Haeckel, 1887, p. 1097, pl. 88, fig. 7; Benson, 1964, pl. 2, figs. 2-3; 1966, p. 293, pl. 20, figs. 2-7.

Amphispyris reticulata (Ehrenberg), Nigrini, 1967, p. 44, pl. 5, fig. 3. Amphispyris costata Haeckel, Nigrini, 1967, p. 45, pl. 5, fig. 4.

Liriospyris reticulata (Ehrenberg), Goll, 1968, p. 1429, pl. 176, figs. 9, 11, 13; Molina-Cruz, 1977, p. 336, pl. 6, fig. 6.

Amphispyris costata-thorax Haeckel group, Casey, 1971, pl. 23.2, figs. 5-7.

Liriospyris sp., Renz, 1976, p. 167, pl. 8, fig. 14.

Liriospyris(?) toxarium A, Molina-Cruz, 1977, p. 336, pl. 6, figs. 1-3.

Liriospyris toxarium (Haeckel), Molina-Cruz, 1977, p. 336, pl. 6, figs. 4-5.

\section{Litharachnium tentorium Haeckel}

Litharachnium tentorium Haeckel, 1860 , p. $836 ; 1862$, p. 281 , pl. 4 , figs. 7-10; Benson, 1966, p. 427, pl. 29, figs. 5-6; Renz, 1974, p. 793, pl. 17, fig. 19; 1976, p. 150, pl. 7, fig. 6; Kling, 1979, p. 309, pl. 1 , fig. 22.

\section{Lithelius minor Jörgensen}

Lithelius minor Jörgensen, 1899, p. 65 , pl. 5, fig. 24; Benson, 1964 , pl. 1 , fig. 38 ; 1966 , p. 262 , pl. 17 , figs. $9-10$, pl. 18 , figs. $1-4$; Kling, 1977, p. 217, pl. 1, fig. 16.

\section{Lithelius(?) sp.}

Lithelius? sp., Benson, 1964, pl. 1, fig. 37; 1966, p. 265, pl. 18, figs. 5-6.

Spongurus(?) sp., Ling, Stadum, and Welch, 1971, p. 711, pl. 1, fig. 6 ; Kling, 1977, p. 217, pl. 2, fig. 3.

Spongurus sp., Molina-Cruz, 1977, p. 333, pl. 1, fig. 2.

Remarks. As noted by Benson (1966, p. 265), this species has an internal structure similar to that of Lithelius minor, i.e., four to five closely spaced, concentric, trizonal shells which appear as single or double spirals or as concentric shells, depending upon the orientation of the test. On the other hand, the genus Spongurus is more closely allied with species consisting of closely spaced, concentric ellipsoidal (not trizonal) shells such as Spongocore puella and Spongurus sp. cf. S. elliptica.

\section{Lithomelissa hystrix Jörgensen}

Lithomelissa hystrix Jörgensen, 1899, p. 83; 1905, p. 136, pl. 16, fig. 84 ; Benson, 1966 , p. 363 , pl. 24 , figs. $6-8,9(?)$.
Lithomelissa cf. thoracites Haeckel, Dumitrică, 1972, p. 837, pl. 21, figs. 14-15.

Arachnocorys(?) sp. cf. A. pentacantha Popofsky, Kling, 1977, p. 215 , pl. 1, fig. 10 .

\section{Lithomelissa laticeps Jörgensen \\ (Plate 9, Fig. 3)}

Lithomelissa laticeps Jörgensen, 1905, p. 136, pl. 16, fig. 84; Benson, 1966 , p. 369 , pl. 24 , figs. $14-15$.

\section{Lithomelissa monoceras Popofsky}

Lithomelissa thoracites Haeckel, Benson, 1966, p. 366, pl. 24, fig. 13 (not figs. 10-12).

Lithomelissa monoceras Popofsky, Casey, 1971, p. 23.2, fig. 16; Renz, 1974, p. 794, pl. 18, fig. 14; 1976, p. 158, pl. 6, fig. 12.

\section{Lithomelissa thoracites Haeckel}

(Plate 9, Fig. 2)

Lithomelissa thoracites Haeckel, 1862, p. 301, pl. 6, figs. 2-8; Benson, 1964, pl. 2, fig. $17 ; 1966$, p. 366 , pl. 24 , figs. 10-12 (not fig. 13).

\section{Lithopera bacca Ehrenber}

Lithopera bacca Ehrenberg, Benson, 1966, p. 489, pl. 33, fig. 10-11; Nigrini, 1967 , p. 54 , pl. 6 , fig. 2 .

\section{Lithostrobus sp. cf. L. hexagonalis Haeckel}

Cf. Lithostrobus hexagonalis Haeckel, p. 1475, pl. 79, fig. 20; Nigrini, 1968 , p. 58 , pl. 1 , fig. 10

Lithostrobus cf. hexagonalis Haeckel, Benson, 1964, pl. 2, fig. 61; 1966 , p. 508 , pl. 35 , figs. $1-2$.

\section{Lophocorys polyacantha Popofsky group}

Lophocorys polyacantha Popofsky, 1913, p. 400, fig. 122; Benson, 1966, p. 494, pl. 34, figs. 1-3; Kling, 1979, p. 309, pl. 1, fig. 27.

(?)Artopilium undulatum Popofsky, 1913 , p. 405 , pl. 36, figs. 4-5; Renz, 1974, p. 788 , pl. 16, fig. 13.

(?)Stichopilium anocor Renz, 1976, p. 124, pl. 5, fig. 10.

Remarks. This group is characterized by undulatory constrictions in the distally expanding abdomen and by a relatively large cephalis. Variations in members of this group are the number of abdominal constrictions, the size and shape of pores, and the degree of spininess of the cephalis (smooth surface to one with several scattered, thin, conical spines; with or without apical horn).

\section{Lophophaena cylindrica (Cleve)}

Acanthocorys variabilis Popofsky, Benson, 1964, pl. 2, fig. 14; 1966, p. 373 , pl. 24 , fig. 19 .

Lophophaena cylindrica (Cleve), Renz, 1974, p. 794 , pl. 18, fig. 6 ; 1976 , p. 159 , pl. 6 , fig. 21.

\section{Lophophaenoma sp. aff. L. witjazii Petrushevskaya}

Lophophaena cf. capito Ehrenberg, Benson, 1964, pl. 2, fig. 16; 1966, p. 378, pl. 24 , figs. $22-23$, pl. 25 , fig. 1 .

Lophophaenoma sp. aff. L. witjazii Petrushevskaya, Renz, 1974, p. 794 , pl. 18 , fig. $13 ; 1976$, p. 159 , pl. 6 , fig. 14 .

\section{Lophospyris pentagona (Ehrenberg) hyperborea (Jörgensen)}

Ceratospyris polygona Haeckel, Benson, 1966, p. 321, pl. 22, figs. 1718 (not figs, 15-16)

Lophospyris pentagona hyperborea (Jörgensen), Goll, 1976, p. 400, pl. 14 , figs. $4-6,8-9,11-12$, pl. 15 .

\section{Lophospyris pentagona pentagona (Ehrenberg)}

Certospyris polygona Haeckel, Benson, 1964, pl. 2, fig. 10; 1966, p. 321 , pl. 22, figs. 15-16 (not figs, 17-18).

Lophospyris pentagona pentagona (Ehrenberg), Goll, 1976, p. 398, pl. 10, pl. 11, figs. 1-3, 5 . 


\section{Lophospyris pentagona (Ehrenberg) quadriforis (Haeckel)}

Ceratospyris cf. pentagona Ehrenberg, Benson, 1966, p. 324, pl. 22, figs. 19-21.

Lophospyris pentagona quadriforis (Haeckel), Goll, 1976, p. 398, pl. 13 , pl. 14 , figs. $1-3,7,10,13$.

\section{Neosemantis distephanus (Haeckel)}

Semantis distephanus Haeckel, 1887, p. 957, pl. 83, fig. 3.

Neosemantis distephanus Popofsky, 1913, p. 299, pl. 29, fig. 2.

Neosemantis distephanus (Haeckel) Popofsky, Benson, 1966, p. 291, pl. 19, fig. 18, pl. 20, fig. 1.

Neosemantis distephanus (Haeckel), Kling, 1979, p. 309, pl. 1, figs. $15-16$.

\section{Nephrospyris renilla Haeckel}

Nephrodictyum renilla (Haeckel), Benson, 1966, p. 302, pl. 21, fig. 5. Nephrospyris renilla Haeckel, Dumitrică, 1972, p. 841, pl. 28, fig. 11; Renz, 1974, p. 794, pl. 19, fig. 6; 1976, p. 176, pl. 8, fig. 18.

\section{Octopyle stenozona Haeckel}

Octopyle stenozona Haeckel, 1887, p. 652, pl. 9, fig. 11; Benson, 1964, pl. 1, fig. 53; 1966, p. 251, pl. 16, figs. 3-4; Molina-Cruz, 1977 , p. 335 , pl. 5 , figs. $1-3$.

\section{Ommatartus tetrathalamus (Haeckel)}

Zygocampe chrysalidium Haeckel, Benson, 1964, pl. 1, figs. 5, 22-25; 1966, p. 193 , pl. 8 , figs. $8-13$, pl. 9, figs. 1-2, text-fig. 10.

Panartus tetrathalamus Haeckel, Nigrini, 1967, p. 30, pl. 2, figs. 4a-d.

Panartus tetrathalamus tetrathalamus Haeckel, Nigrini, 1970, p. 168, pl. 1 , fig. 12.

Ommatartus tetrathalamus (Haeckel), Riedel and Sanfilippo, 1971, p. 1588, pl. 1C, figs. 5-7; Kling, 1977, p. 217, pl. 2, fig. 11.

\section{Peridium longispinum Jörgensen(?)}

Peridium sp., Benson, 1966, p. 362, pl. 24, figs. 4-5

(?)Peridium longispinum Jörgensen, 1905, p. 135, pl. 15, figs. 75-79, pl. 16, fig. 80; Bjørklund in Aarseth et al., 1975, p. 58, fig. 14, Radiolarians-F.

\section{Peridium spinipes Haeckel}

Peridium longispinum Jörgensen, Benson, 1966, p. 359, pl. 23, fig. 27, pl. 24 , figs. $1-3$.

Peridium spinipes Haeckel, Casey, 1971, pl. 23.2, figs. 17-18.

Psilomelissa calvata Haeckel, Renz, 1974, p. 795, pl. 18, fig. 8; 1976, p. 160, pl. 6 , fig. 15 .

\section{Peripyramis circumtexta Haeckel}

Peripyramis circumtexta Haeckel, 1887, p. 1162, pl. 54, fig. 5; Riedel 1958 , p. 231 , pl. 2 , figs. $8-9$; Benson, 1966 , p. 426 , pl. 29 , fig. 4 ; Kling, 1973, p. 637, pl. 2, figs. 15-19, pl. 9, figs. 1-3; Kling, 1979, p. 309, pl. 1, fig. 20.

\section{Phormacantha hystrix Jörgensen (Plate 7, Fig. 12)}

Phormacantha hystrix Jörgensen, 1905, p. 132, pl. 14, figs. 59-63; Benson, 1966, p. 357, pl. 23, figs. 24-26.

\section{Phormospyris stabilis (Goll) capoi Goll}

Rhodospyris sp., Benson, 1964, pl. 2, fig. 9; 1966, pl. 23, figs. 3-5. Phormospyris stabilis capoi Goll, 1976, p. 392, pl. 5, figs. 1-2, pl. 6, pl. 7.

\section{Phormospyris stabilis (Goll) scaphipes (Haeckel)}

Tristylospyris scaphipes Haeckel, Benson, 1964, pl. 2, figs. 7-8; 1966, p. 316, pl. 22, figs. 7-10, text-fig. 8A; Casey, 1971, pl. 23.2, figs. 19-20.

Tholospyris scaphipes (Haeckel), Goll, 1969, p. 328, pl. 58, figs. 1-8, 13,14 .
Phormospyris stabilis scaphipes (Haeckel), Goll, 1976, p. 394, pls. $8,9$.

Phormospyris scaphipes (Haeckel), Kling, 1979, p. 309, pl. 1, fig, 17.

\section{Phormospyris stabilis stabilis (Goll)}

Desmospyris anthocyrtoides (Bütschli), Benson, 1964, pl. 2, fig. 11; 1966 , p. 332 , pl. 23 , figs. $6-8$.

Phormospyris stabilis stabilis (Goll), Goll, 1976, p. 390, pl. 1, pl. 2, figs. 7-14.

\section{Phormospyris tricostata Haeckel}

Phormospyris tricostata Haeckel, 1887, p. 1087, pl. 83, fig. 15; Benson, 1964, pl. 2, fig. 12; 1966, p. 334, pl. 23, fig. 9.

\section{Phormostichoartus corbula (Harting)}

Siphocampium cf. polyzona Haeckel, Benson, 1964, pl. 2, fig. 49; 1966 , p. 513 , pl. 35 , figs. $5-8$, text-fig. 27.

Siphocampe corbula (Harting), Nigrini, 1967, p. 85, pl. 8, fig. 5, pl. 9, fig. 3; 1970, p. 172, pl. 4, fig. 11; Riedel and Sanfilippo, 1971, p. 1601, pl. 1H, figs. 18-25; Kling, 1973, p. 639, pl. 5, figs. 22-23, pl. 12, figs. 21-23(?); Molina-Cruz, 1977, p. 338, pl. 8, fig. 6

Phormostichoartus corbula (Harting), Nigrini, 1977, p. 252, pl. 1, fig. 10; Kling, 1979, p. 309, pl. 2, fig. 20.

\section{Phorticium pylonium Haeckel group} (Plate 7, Figs. 15-16)

Phorticium pylonium Haeckel, 1887, p. 709, pl. 49, fig. 10.

Phorticium pylonium (Haeckel?) Cleve, Riedel, 1958, p. 229, pl. 2, fig. 5 ; Benson, 1964 , pl. 1 , fig. 61 ; 1966, p. 252 , pl. 16, figs. 5-9, pl. 17 , figs. 1-3.

Pylospira octopyle Haeckel(?), Nigrini and Moore, 1979, p. S139, pl. 17 , figs. $6 a-c$.

Remarks. This group differs from the Tetrapyle octacantha group in (1) the presence of more than three (as many as five) systems of dimensive girdles supported by numerous radial beams (20-30) not confined to the regions of the dimensive axes of the test, and (2) the presence, in fully-developed individuals, of an ellipsoidal outer shell of smooth outline (Benson, 1966, pl. 17, figs. 1-3; this chapter, Plate 7, Fig. 15) similar to the outer shell of Hexapyle dodecantha and Larcopyle bütschlii. This group is a difficult one to work with because in certain orientations individuals appear as a double spiral (Benson, 1964, pl. 1, fig. $61 ; 1966$, pl. 16, figs. 5, 8) and in others as a concentric system of elliptical shells (Benson, 1966, pl. 16, fig. 9; this chapter, Plate 7, Fig. 16).

\section{Plagiacantha(?) panarium Dumitricà} (Plate 7, Figs. 10-11)

Plectacantha? sp., Benson, 1966, p. 356, pl. 23, figs. 21-23. Plagiacantha(?) panarium Dumitricǎ, 1972, p. 835 , pl. 22, figs. 1, 3,5 .

\section{Plagonium sp. cf. $P$. sphaerozoum Haeckel} (Plate 7, Figs. 1-2)

Cf. Plagonium sphaerozoum Haeckel, 1887, p. 916, pl. 91, fig. 6 . Plagonium cf. sphaerozoum Haeckel, Benson, 1966, p. 286, pl. 19, figs. 12-13.

\section{Plectacantha oikiskos Jörgensen}

(Plate 7, Figs. 13-14)

Plectacantha oikiskos Jörgensen, 1905 , p. 131 , pl. 13, figs. 50-57 Benson, 1964, pl. 2, fig. 14(?); 1966, p. 353, pl. 23, figs. 18-20. Remarks. In the Gulf of California, this species(?) may represent tests of Lithomelissa hystrix with the thorax undeveloped.

\section{Plectopyramis dodecomma Haeckel}

Plectopyramis dodecomma Haeckel, 1887, p. 1258, pl. 54, fig. 6; Benson, 1964 , pl. 2, fig. 26 ; 1966, p. 424 , pl. 29, fig. 3.

Bathropyramis woodringi Campbell and Clark, Kling, 1973, p. 635 , pl. 2, figs. 20-23, pl. 9, figs. 4, 5(?), 6, 7(?).

Peripyramis circumtexta Haeckel, Casey, 1971, pl. 23.1, fig. 11. 
Cinclopyramis infundibulum Haeckel, Renz, 1974, p. 789, pl. 17, fig. 23 ; 1976 , p. 149 , pl. 7 , fig. 12

Remarks. The specimen identified as Plectopyramis dodecomme and illustrated by Nigrini and Moore (1979, pl. 21, fig. 5) appears to represent a different species for the following reasons: (1) the thorax flares distally, producing a more trumpet-like than conical shape, (2) the transverse bars of the thoracic meshwork are not continuous around the circumference, and (3) the thoracic surface has scattered spines. Their specimen is probably conspecific with one illustrated by Renz (1976, pl. 7, fig. 3) that she identified as Bathropyramis sp.

\section{Pseudocubus obeliscus Haeckel}

$$
\text { (Plate 7, Figs. 8-9) }
$$

Pseudocubus obeliscus Haeckel, 1887, p. 1010, pl. 94, fig. 11; Benson, 1966 , p. 312 , pl. 22, figs. 3-6.

Plectophora triacantha Popofsky, 1908, p. 262, pl. 29, fig. 1, pl. 30 fig. 1.

Obeliscus pseudocuboides Popofsky, 1913, p. 280, pl. 29, figs. 4-5.

\section{Pseudodictyophimus gracilipes (Bailey)}

Dictyophimus gracilipes Bailey, Benson, 1966, p. 382, pl. 25, figs. 46.

Pseudodictyophimus gracilipes (Bailey), Kling, 1977, p. 217, pl. 1, fig. 7; 1979, p. 309, pl. 1, figs. 23-24.

\section{Psilomelissa(?) sp. cf. P.(?) galeata Ehrenberg}

Psilomelissa galeata Ehrenberg(?), Popofsky, 1908, p. 304, pl. 33, fig. 6 .

Lithomelissa cf. galeata (Ehrenberg)? Popofsky, Benson, 1964, pl. 2 fig. $34 ; 1966$, p. 371 , pl. 24 , figs. 16-17, 18(?).

Remarks. This species appears to be related to Lithomelissa spp. but lacks the well-developed lateral spines of this genus.

\section{Pterocanium bicorne Haeckel(?)}

Pterocanium sp., Benson, 1964, pl. 2, fig. 21; 1966, p. 401, pl. 26 , figs. 5-6; Casey, 1971, pl. 23.1, figs. 1-2; Nigrini and Moore, 1979 , p. N49, pl. 23, figs. 6a-b.

(?)Pterocanium bicorne Haeckel, Renz, 1974, p. 795, pl. 17, fig. 6 .

Dictyophimus infabricatus, Molina-Cruz, 1977, pl. 8, fig. 1.

Remarks. The assignment of this species to Pterocanium bicorne is questionable in light of Nigrini and Moore's (1979) reservations about applying Haeckel's species name before examining topotypic material.

\section{Pterocanium grandiporus Nigrini}

Pterocanium grandiporus Nigrini, 1968, p. 57, pl. 1, fig 7; Benson, 1964, pl. 2, fig. 39; Molina-Cruz, 1977, p. 336, pl. 6, fig. 11.

\section{Pterocanium korotnevi (Dogiel)}

Pterocanium korotnevi (Dogiel), Benson, 1964, pl. 2, fig. 18; Nigrini, 1970, p. 170, pl. 3, figs. 10-11; Ling, Stadum, and Welch, 1971, p. 714 , pl. 2, fig. 4 ; Kling, 1973, p. 638 , pl. 4, figs. 1-4.

\section{Pterocanium praetextum (Ehrenberg) eucolpum Haeckel}

Pterocanium prosperinae Ehrenberg, Benson, 1964, pl. 2, fig. 38(?) 1966 , p. 405 , pl. 27 , figs. $3(?$ ), 5 (not fig. 4 ).

Pterocanium praetextum (Ehrenberg) eucolpum Haeckel, Nigrini, 1967, p. 70, pl. 7, fig. 2; 1970, p. 170, pl. 3, fig. 8; Kling, 1979, p. 311 , pl. 2, figs. 14a-b, 15a-b, 16.

\section{Pterocanium praetextum praetextum (Ehrenberg)}

Pterocanium praetextum (Ehrenberg), Benson, 1964, pl. 2, fig. 22; 1966 , p. 408 , pl. 27 , fig. 6 , pl. 28 , fig. 1 .

(?)Pterocanium prosperinae Ehrenberg, Benson, 1964, pl. 2, fig. 38; 1966 , p. 405 , pl. 27 , fig. 3 (not figs 4,5 ).

Pterocanium praetextum praetextum (Ehrenberg), Nigrini, 1967, p. 68 , pl. 7 , fig. $1 ; 1970$, p. 170 , pl. 3 , fig. 7.

\section{Pterocanium prismatium Riedel}

Pterocanium prismatium Riedel, 1957, p. 87, pl. 3, figs. 4-5; Riedel and Sanfilippo, 1971, p. 1595, pl. 8, fig. 1; Nigrini, 1971, p. 447, pl. 34.1, fig. 4; Johnson and Knoll, 1975, p. 109, pl. 1, fig. 9.
Remarks. This useful stratigraphic marker for the top of the Pliocene was not found in sediments that may be of Pliocene age at Site 485 .

\section{Pterocanium sp. cf. P. elegans (Haeckel)}

Cf. Artopilium elegans Haeckel, 1887, p. 1440, pl. 75, fig. 1. Pterocanium cf. elegans (Haeckel), Benson, 1966, p. 403, pl. 27, figs. $1-2$.

Eucyrtid 2 gen. and sp. indet., Renz, 1974, p. 791, pl. 17, fig. 1.

\section{Pterocanium trilobum (Haeckel)}

Pterocanium prosperinae Ehrenberg, Benson, 1964, pl. 2, fig. 19; 1966, p. 405 , pl. 27, fig. 4 (not figs. 3, 5).

Lychnodictyum challengeri Haeckel, Benson, 1966, p. 410, pl. 28, figs. $2-3$.

Pterocanium trilobum Haeckel, Nigrini, 1967, p. 71, pl. 7, figs. 3a-b; 1970 , p. 170, pl. 3, fig. 9; Casey, 1971, pl. 23.3 fig. 14; Kling, 1973 , p. 638 , pl. 4 , figs. $5-8$; Molina-Cruz, 1977 , p. 337 , pl. 8 , fig. 5 .

\section{Pterocorys hertwigii (Haeckel)}

Phormocyrtis fastuosa (Ehrenberg), Benson, 1966, pl. 33, figs. 6-7. Theoconus hertwigii (Haeckel), Nigrini, 1967, p. 73, pl. 7, figs. 4a-b; Molina-Cruz, 1977 , p. 338 , pl. 8 , figs. 7-8

Eucyrtidium hertwigii Haeckel, Casey, 1971, pl. 23.1, figs. 18-20.

Pterocorys hertwigii (Haeckel), Riedel and Sanfillppo, 1978, p. 72, pl. 9, fig. 2; Nigrini and Moore, 1979, p. N85, pl. 25, fig. 9.

\section{Pterocorys killmari (Renz)}

Pterocorys cf. columba Haeckel, Benson, 1964, pl. 2, fig. 35; 1966, p. 414 , pl. 28 , fig. 7.

Corocalyptra killmari Renz, 1974, p. 790 , pl. 17, fig. 10;1976, p. 118, pl. 4, fig. 11.

Remarks. Because its test is "more cylindrical, very different from other hat-shaped Corocalyptra"' (Renz, 1976, p. 118), I have placed this species in the genus Pterocorys (cephalis, thorax, abdomen with three solid thoracic wings; without terminal feet, Campbell, 1954, p. 130).

\section{Pterocorys minythorax (Nigrini)}

Theoconus zancleus (Müller), Benson, 1964, pl. 2, figs. 50-51; 1966, p. 482 , pl. 33, fig. 5 (not fig. 4); Casey, 1971, pl. 23.3, fig. 15.

Theoconus minythorax Nigrini, 1968, p. 57, pl. 1, fig. 8; Kling, 1977, p. 217 , pl. 1, fig. 8

Pterocorys zancleus (Müller), Casey, 1977, pl. 4, fig. 15.

\section{Pterocorys zancleus (Müller)}

Theoconus zancleus (Müller), Benson, 1966, p. 482, pl. 33, fig. 4 (not fig. 5).

(?)Pterocorys clausus (Popofsky), Kling, 1979, p. 311, pl. 2, fig. 22. Pterocorys zancleus (Mueller), Nigrini and Moore, 1979, pl. 25, figs. $11 \mathrm{a}-\mathrm{b}$.

Pylonium sp.

(Plate 6, Fig. 5)

Pylonium sp., Benson, 1966, p. 250, pl. 16, fig. 2.

\section{Saturnalis circularis Haeckel}

Saturnalis circularis Haeckel, Nigrini, 1967, p. 25, pl. 1, fig. 9; Kling, 1973, p. 635 , pl. 1, figs. 21-25, pl. 7, figs. 1-5.

\section{Sethoconus(?) dogieli Petrushevskaya}

Sethoconus(?) dogieli Petrushevskaya, Dumitrică, 1972, p. 837, pl. 23, figs. 1-2.

Lipmanella(?) dogieli (Petrushevskaya), Petrushevskaya and Kozlova, 1972 , p. 542 , pl. 37 , fig. 10.

\section{Sethophormis pentalactis Haeckel}

Lampromitra cf. coronata Haeckel, Benson, 1966, p. 452, pl. 30, fig. 8 (not figs. 9-10). 
Sethophormis pentalactis Haeckel, Renz, 1974, p. 795, pl. 18, figs. 18a-b; 1976, p. 165, pl. 7, fig. 7 .

\section{Sethophormis sp. aff. $S$. pentalactis Haeckel}

Lampromitra cf. coronata Haeckel, Benson, 1966, p. 452, pl. 30, figs. 9-10 (not fig. 8).

Sethophormis sp. aff. S. pentalactis Haeckel, Renz, 1974, p. 795, pl. 18 , fig. 22.

\section{Siphocampe arachnea (Ehrenberg) group}

Siphocampe arachnea (Ehrenberg) group, Nigrini, 1977, p. 255, pl. 3, figs. 7-8; Kling, 1979, p. 311, pl. 2, fig. 19.

Lithomitra lineata (Ehrenberg), Kling, 1977, p. 217, pl. 1, fig. 1.

\section{Siphocampe lineata (Ehrenberg) group}

Siphocampium cf. cylindrica Haeckel, Benson, 1964, pl. 2, fig. 48; 1966 , p. 520 , pl. 35 , figs. $10-11$.

Siphocampe lineata (Ehrenberg) group, Nigrini, 1977, p. 256, pl. 3, figs. 9-10.

\section{Siphocampe nodosaria (Haeckel)}

Siphocampe nodosaria (Haeckel), Nigrini, 1977, p. 256, pl. 3, fig. 11.

\section{Siphocampe sp.}

Siphocampium sp., Benson, 1964, pl. 2, fig. 47; 1966, p. 517, pl. 35, fig. 9.

\section{Siphonosphaera polysiphonia Haeckel}

Siphonosphaera cf. socialis Haeckel, Benson, 1966, p. 121, pl. 2, fig. 4 .

Siphonosphaera polysiphonia Haeckel, Nigrini, 1967, p. 18, pl. 1, figs. 4a-b; 1970, p. 167, pl. 1, fig. 6; Kling, 1979, p. 311, pl. 1, fig. 1.

\section{Sphaeropyle langii Dreyer}

Sphaeropyle langii Dreyer, 1889, p. 89, pl. 9, fig. 54; Benson, 1966, p. 166, pl. 5, figs. 7-9; Kling, 1973, p. 634, pl. 1, figs. 5-10, pl. 13, figs. 6-8.

(?)Prunopyle antarctica Dreyer, 1889, p. 24, pl. 5, fig. 75; Riedel, 1958 , p. 225 , pl. 1 , figs. $7-8$.

\section{Spirema sp.}

(Plate 6, Figs. 3-4)

Spirema sp., Benson, 1966, p. 268, pl. 18, figs. 9-10.

\section{Spirocyrtis gyroscalaris Nigrini}

Siphocampium cf. cornutella Haeckel, Benson, 1966, p. 523, pl. 35, figs. 14-15 (not figs. 16-17).

Spirocyrtis gyroscalaris Nigrini, 1977, p. 258, pl. 2, figs. 10-11.

\section{Spirocyrtis scalaris Haeckel}

Siphocampium cf. cornutella Haeckel, Benson, 1966, p. 523, pl. 35, figs. 16-17 (not figs. 14-15).

Spirocyrtis scalaris Haeckel, Nigrini, 1967, p. 88, pl. 8, fig. 7, pl. 9, figs. $4 ; 1977$, p. 259 , pl. 2 , figs. $12-13$.

\section{Spongaster tetras Ehrenberg}

Spongaster tetras Ehrenberg, Benson, 1964, pl. 1, fig. 46; 1966; p. 238, pl. 15, fig. 2; Riedel and Sanfilippo, 1971, p. 1589, pl. 1D, figs. 5-7; Casey, 1971, pl. 23.3, figs. 18-19.

Spongaster tetras tetras Ehrenberg, Nigrini, 1967, p. 41, pl. 5, figs. la-b; 1970, p. 169, pl. 2, fig. 7.

\section{Spongocore puella Haeckel}

Spongocore puella Haeckel, Benson, 1964, pl. 1, figs. 21, 43, 44; 1966 , p. 187 , pl. 8 , figs. $1-3$; Nigrini, 1970 , p. 168 , pl. 2 , fig. 3 ; Casey, 1971, pl. 23.3, fig. 20; Kling, 1977, pl. 2, fig. 12.

Spongocore diplocylindrica Haeckel, Renz, 1976, p. 95, pl. 3, fig. 8 .

\section{Spongodiscus biconcavus (Haeckel)}

Spongodiscus biconcavus (Haeckel), Popofsky, 1912, p. 143, pl. 6, fig. 2; Benson, 1964, pl. 1, fig. 42; 1966, p. 214, pl. 11, fig. 1 , text-fig. 14.

Spongaster disymmetricus (Dogiel), Petrushevskaya and Kozlova, 1972 , p. 528 , pl. 21 , fig. 14.

Spongodiscus sp. 3, Renz, 1974, p. 796, pl. 15, fig. 11.

\section{Spongopyle osculosa Dreyer}

Spongopyle osculosa Dreyer, Benson, 1966, p. 215, pl. 11, figs. 2-3, text-fig. 15; Casey, 1971, pl. 23.1, fig. 14; Kling, 1977, p. 217, pl. 1 , fig. 4 .

\section{Spongosphaera streptacantha Haeckel}

Spongosphaera streptacantha Haeckel, 1860, p. 840, 1862, p. 455, pl. 26, figs. 1-3; Benson, 1966, p. 175, pl. 6, fig. 4, pl. 7, fig. 1; Renz, 1976, p. 105 , pl. 2, fig. 13.

\section{Spongotrochus sp. cf. S. glacialis Popofsky} (Plate 5, Fig. 3)

Cf. Spongotrochus glacialis Popofsky, 1908, p. 228, pl. 26, fig. 8, pl. 27, fig. 1, pl. 28, fig. 2; Casey, 1971, pl 23.1, figs. 4-5.

Spongotrochus cf. glacialis Popofsky, Benson, 1966, p. 218, pl. 11, fig. 4 , text-fig. 16 .

\section{Spongurus sp. cf. S. elliptica (Ehrenberg)}

Cf. Acanthosphaera elliptica Ehrenberg, 1872a, p. 301; 1872b, pl. 7, fig. 4 .

Spongurus cf. elliptica (Ehrenberg), Benson, 1966, p. 189, pl. 8, figs. 4-5; Nigrini and Moore, 1979, p. S63, pl. 8, fig. 2.

\section{Stichopera pectinata Haeckel group}

Cyrtopera laguncula Haeckel, Benson, 1966, p. 510, pl. 35, figs. 3-4; Casey, 1971, pl. 23.1, fig. 10.

Stichopera pectinata Haeckel group, Kling, 1973, p. 638, pl. 3, figs. $25-27$, pl. 10 , figs. $1-5 ; 1979$, p. 311 , pl. 1 , fig. 19.

\section{Stichopilium bicorne Haeckel}

Stichopilium bicorne Haeckel, 1887, p. 1437, pl. 77, fig. 9; Benson, 1964 , pl. 2, fig. 36; 1966, p. 422, pl. 29, figs. 1-2; Renz, 1976, p. 125, pl. 4, fig. 9; Molina-Cruz, 1977, p. 337, pl. 7, fig. 14; Kling, 1979 , p. 311 , pl. 2 , figs. $11-12$.

Corocalyptra sp. aff. C. kruegeri Popofsky, Renz, 1974, p. 790, pl. 16 , fig. 11.

\section{Stylacontarium acquilonium (Hays)}

Druppatractus acquilonius Hays, 1970, p. 214, pl. 1, figs. 4-5. Stylacontarium acquilonium (Hays), Kling, 1973, p. 634, pl. 1, figs. $17-20$, pl. 14, figs. $1-4$.

Remarks. Although I searched for $S$. acquilonium in each of the Leg 65 samples I examined, I found only specimens of $S$. bispiculum, these latter do not have an outer medullary shell of elliptical shape protruding at the connecting bars, characters which define $S$. acquilonium (Kling, 1973, p. 634).

\section{Stylacontarium bispiculum Popofsky (Plate 1, Figs. 7-10)}

Stylacontarium bispiculum Popofsky, Kling, 1973, p. 634, pl. 15, figs. 11-14.

Remarks. As noted by Kling (1973, p. 634), this species differs from $S$. acquilonium ( = Druppatractus acquilonius) primarily in having a spherical outer medullary shell. Based on this criterion, no specimens of $S$. acquilonium are present in Leg 65 samples, but $S$. bispiculum ranges throughout the Quaternary section cored.

\section{Stylacontarium sp. ef. S. bispiculum Popofsky}

Cf. Stylacontarium bispiculum Popofsky, 1912, p. 91, pl. 2, fig. 2. Stylacontarium bispiculum Popofsky, Benson, 1964, pl. 1, figs. 8, 11; 1966 , p. 141 , pl. 3, figs. 8-11. 
Remarks. Benson (1966) characterized this species as a cubosphaerid having a cortical shell with a subquadrate outline and compressed in one dimensive axis. It differs from Popofsky's species in having three-bladed rather than conical main spines and a thorny to spiny rather than smooth cortical shell. The latter characteristics agree well with Kling's (1973, pl. 15, figs. 13-14) illustrations of thin-walled specimens of $S$. bispiculum.

\section{Stylochlamydium asteriscus Haeckel group}

Ommatodiscus sp., Benson, 1964, pl. 1, fig. 35; 1966, p. 210, pl. 10, figs. 3, 5(?), 6 .

Stylochlamydium sp. aff. S. venustum (Bailey), Renz, 1974, p. 798, pl. 15 , fig. 17.

Stylochlamydium asteriscus Haeckel, Molina-Cruz, 1977, p. 335, pl.

4, fig. 6; Nigrini and Moore, 1979, p. S113, pl. 14, fig. 5.

Remarks. This group includes specimens with an opaque, biconvex central region (concentric discoidal shells), surrounded in a single plane by numerous concentric, equally spaced, regular, latticed rings covered by a porous sieve plate and with or without marginal spines. Nigrini and Moore (1979, p. S107) designate an incompletely developed test of S. asteriscus illustrated by Benson (1966, pl. 10, fig. 3 ) as Porodiscus sp. A.

\section{Stylochlamydium venustum (Bailey) group}

Ommatodiscus sp., Benson, 1964, pl. 1, fig. 40, fig. 36(?); 1966, p. 210 , pl. 10, figs. 2, 4, 5(?), 7.

Stylochlamydium venustum (Bailey), Kling, 1977, p. 217, pl. 1, fig. 5 .

Stylodictya sp. aff. S. multispina Haeckel, Renz, 1974, p. 798, pl. 15, fig. 12 .

Remarks. This group includes specimens with an opaque, biconvex central region (concentric discoidal shells), surrounded in a single plane by generally irregular concentric rings which may be broken into concave outward segments near the periphery of the test. A porous sieve plate covers the rings on both sides of the test. Nigrini and Moore (1979, p. S109) identify an incompletely developed specimen of $S$. venustum illustrated by Benson (1966, pl. 10, fig. 4) as Porodiscus(?) sp. B.

\section{Stylodictya validispina Jörgensen}

Stylodictya validispina Jörgensen, Benson, 1964, pl. 1, fig. 35; 1966, p. 203, pl. 9, figs. 5-6, text-fig. 11; Kling, 1977, p. 217, pl. 2, fig. 1. Xiphospira sp. cf. X. circularis (Clark and Campbell), Kling, 1973, p. 635 , pl. 2, figs. 1-3, pl. 7, figs. 11-14 (not figs. 15-17). Stylodictya multispina Haeckel, Renz, 1976, p. 111, pl. 3, fig. 13.

\section{Tessarastrum straussi Haeckel}

Tessarastrum straussi Haeckel, Renz, 1974, p. 798, pl. 15, fig. 15; 1976, p. 112, pl. 3, fig. 7.

Amphirhopalum cf. Tessarastrum straussi Haeckel, Johnson and Nigrini, 1980, p. 148, pl. 2, fig. 4(?), pl. 5, fig. 1, 2(?).

Remarks. The rarely occurring tests of this species in Leg 65 samples show no indication of cross arms (as illustrated by Johnson and Nigrini, 1980 , pl. 5, fig. 1). Renz (1974, p. 798) noted that the cross arms were either rudimentary or lacking. Johnson and Nigrini (1980) suggest that the lateral arms are taxonomically unimportant; therefore, the species might be more properly placed in the genus $\mathrm{Am}$ phirhopalum.

\section{Tetrapyle octacantha Müller group}

Tetrapyle octacantha Müller, 1858, p. 33, pl. 2, figs. 12-13, pl. 3, figs. 1-12; Benson, 1964, pl. 1, figs. 48-52, 54-59; 1966, p. 245, pl. 15 , figs. $3-10$, pl. 16, fig. 1, text-fig. 18; Molina-Cruz, 1977, p. 335, pl. 5, figs. 5-7.

Tetrapyle sp. cf. T. octacantha Muller, Kling, 1977, p. 217, pl. 1, fig. 14 .

Remarks. This group differs from the Phorticium pylonium group in (1) the presence of few, if any, radial beams, which are generally confined to the regions of the dimensive axes of the tests, and (2) the presence, in fully developed individuals, of no more than three systems of girdles, the third being irregular and joined to the second system by numerous short beams arising from the surface of the latter (Benson, 1964, pi. 1, fig. 48; 1966, pl. 16, fig. 1).

\section{Thecosphaera spp.}

Thecosphaera sp., Benson, 1964, pl. 1, figs. 2-4; 1966, p. 132, pl. 2, figs. 11-13.

(?)Cenosphaera spp., Nigrini and Moore, 1979, p. S43, pl. 4, figs. 3a-d.

Remarks. Because many of the specimens in the Gulf of California lack the two medullary shells whereas others with identical cortical shells have these, I have placed all such forms in the genus Thecosphaera (Benson, 1966, p. 133). Nigrini and Moore's (1979, pl. 4, figs. 3a-d) illustrations of Cenosphaera spp. are identical with the Gulf of California specimens and on further study may be found to contain two inner medullary shells.

This group may be subdivided on the basis of size and number of pores of the cortical shell.

Theocalyptra davisiana (Ehrenberg) cornutoides (Petrushevskaya)

Theocalyptra davisiana (Ehrenberg), Benson, 1966, p. 441, pl. 29, fig. 16 (not figs. 14-15); Kling, 1973, p. 441, pl. 3, figs. 5-8.

Cycladophora davisiana "var." cornutoides Petrushevskaya, Ling, Stadum, and Welch, 1971 , p. 714, pl. 2, figs. 6-7.

(?)Clathrocycloma davisiana (Ehrenberg), Dumitrică, 1972, p. 837, pl. 24 , fig. 7 .

Theocalyptra davisiana (Ehrenberg) cornutoides (Petrushevskaya) Kling, 1977, p. 217, pl. 1, fig. 20;1979, p. 311, pl. 2, fig. 3.

\section{Theocalyptra davisiana davisiana (Ehrenberg)}

Theocalyptra davisiana (Ehrenberg), Riedel, 1958, p. 239, pl. 4, figs. 2-3, text-fig. 10; Benson, 1964, pl. 2, figs. 45-46; 1966, p. 441, pl. 29, figs. 14-15 (not fig. 16); Kling, 1973, p. 638, pl. 3, figs. 9-12, 28.

Theocalyptra davisiana davisiana (Ehrenberg), Kling, 1977, p. 217, pl. 2, fig. 17.

Cycladophora davisiana (Ehrenberg), Molina-Cruz, 1977, p. 337, pl. 7 , fig. 19.

\section{Theocorys veneris Haeckel}

Theocorys veneris Haeckel, 1887, p. 1415, pl. 69, fig. 5; Popofsky, 1913 , p. 399 , text-fig. 119 ; Benson, 1966 , p. 492 , pl. 33 , figs. 12-13; Renz, 1976, p. 137, pl. 5, fig. 11; Kling, 1979, p. 313, pl. 2, fig. 9 .

\section{Theocorythium trachelium trachelium (Ehrenberg)}

Calocyclas amicae Haeckel, Benson, 1964, pl. 2, fig. 43; 1966, p. 487, pl. 33 , figs. 8-9.

Theocorythium trachelium trachelium (Ehrenberg), Nigrini, 1967, p. 79 , pl. 8 , fig. 2 , pl. 9 , fig. 2 ; 1970 , p. 172 , pl. 4 , fig. 10; MolinaCruz, 1977, pl. 8, fig. 4.

\section{Theocorythium vetulum Nigrini \\ (Plate 3, Figs. 7-9)}

Theocorythium vetulum Nigrini, 1971 , p. 447 , pl. 34.1 , figs. $6 a-b$; Dinkelman, 1973, p. 788, pl. 10, figs. 11-12; Johnson and Knoll, 1975 , p. 109 , pl. 1 , fig. 8 .

Remarks. Although I searched for $T$. vetulum in Leg 65 samples, I identified only one specimen, in Sample $483-8, C C(11-13 \mathrm{~cm})$. It belongs to the genus Theocorythium because it has paired cephalic lobes beneath the larger, unpaired lobe (Plate 3, Figs. 7, 9), and it has the dimensions of $T$. vetulum (abdominal breadth: $107 \mu \mathrm{m}$; abdominal length: $54 \mu \mathrm{m}$ ). C. Nigrini (personal communication) is not sure that the figured specimen is really $T$. vetulum; if it is, it is atypical.

\section{Theopilium tricostatum Haeckel}

Theopilium tricostatum Haeckel, 1887, p. 1332, pl. 70, fig. 6; Benson, 1964 , pl. 2 , fig. $40 ; 1966$, p. 444 , pl. 30 , figs. $1-2$.

Theocalyptra sp., Renz, 1974 , p. 798 , pl. 16 , fig. $21 ; 1976$, p. 137 , pl. 5, fig. 13.

\section{Tholospyris devexa Goll}

Amphispyris aff. zonarius (Haeckel), Benson, 1966, p. 300, pl. 20, figs. 13-14, pl. 21, figs. 1-4.

Tholospyris devexa Goll, 1969, p. 326, pl. 57, figs. 9, 10, 13, 14. 


\section{Tholospyris kantiana (Haeckel)}

Tricolospyris kantiana Haeckel, Benson, 1966, p. 366, pl. 23, figs. 10-12.

Tholospyris kantiana (Haeckel), Goll, 1969, p. 327, pl. 58, figs. 17$19,23$.

Liriospyris sp. 2, Renz, 1974, p. 793, pl. 19, fig. 4.

\section{Tholospyris procera Goll}

Amphispyris subquadrata Haeckel, Benson, 1964, pl. 2, figs. 1, 13; 1966, p. 297, pl. 20, figs. 8-12.

Tholospyris procera Goll, 1969, p. 328, pl. 59, figs. 8, 10-12.

Trissocyclid gen. and sp, indet.

Petalospyris cf. ophirensis Ehrenberg, Benson, 1966, p. 318, pl. 22, figs. 11-14.

\section{Verticillata hexacantha Popofsky}

Verticillata hexacantha Popofsky, 1913, p. 282, text-fig. 11; Benson, 1966, p. 397, pl. 26, fig. 3; Renz, 1974, p. 799, pl. 18, fig. 1; 1976, p. 161 , pl. 6 , fig. 5 .

\section{Xiphatractus cronos (Haeckel)}

(Plate 4, Fig. 8)

Amphisphaera cronos Haeckel, 1887, p. 144, pl. 17, fig. 5.

Xiphatractus cronos (Haeckel), Benson, 1964, pl. 1, fig. 17; 1966, p. 182, pl. 7, figs. 12-13.

(?)Stylosphaera lithatractus Haeckel, Renz, 1976, p. 105, pl. 2 , fig. 7. Stylactractus spp., Nigrini and Moore, 1979, p. S55, pl. 7, figs. 1a-b.

\section{Xiphatractus pluto (Haeckel) \\ (Plate 5, Figs. 1-2)}

Amphisphaera pluto Haeckel, 1887, p. 144, pl. 17, figs. 7-8.

Xiphatractus pluto (Haeckel), Benson, 1964, pl. 1, fig. 18; 1966, p. 184, pl. 7, figs. 14-17.

(?)Druppatractus (?)sp., Dumitrică, 1972 , p. 833 , pl. 20 , fig. 5 .

\section{Zygocircus productus (Hertwig)}

Zygocircus productus (Hertwig), Benson, 1964, pl. 2, fig. 5; 1966, p. 288, pl. 19, figs. 14-15; Dumitrică, 1972, p. 840, pl. 27, figs. 7-10.

Zygocircus sp. aff. Z. capulosus Popofsky, Renz, 1974, p. 799, pl. 19, fig. $23 ; 1976$, p. 170 , pl. 8 , fig. 2.

\section{Zygocircus sp.}

(Plate 7, Figs. 3-4)

Zygocircus sp., Benson, 1966, p. 290, pl. 19, figs. 16-17.

\section{Sponge Spicules}

\section{Geodia phlegraei (Sollas)(?)}

Spumellina incertae sedis, Forma B, Benson, 1966, p. 284, pl. 19, figs. 9-11.

(?)Geodia phlegraei (Sollas), Aarseth et al., 1975, p. 57, fig. 14. Sponge spicules-C.

Remarks. Geodia phlegraei is a sponge spicule.

\section{Hataina ovata Huang}

Spumellina incertae sedis, Forma A, Benson, 1966, p. 283, pl. 19, figs. 6-8.

Hataina ovata Huang, 1967, p. 178, pl. 17, figs. 1-6, pl. 18, figs. 1-4, pl. 19, figs. 1-6.

Remarks. This may be part of a sponge sketelon (W. R. Riedel, personal communication).

\section{ACKNOWLEDGMENTS}

I thank Cathy Nigrini, La Habra Heights, California, and Ted Moore, Jr., University of Rhode Island, for their reviews of the original manuscript.

\section{REFERENCES}

Aarseth, 1., Bjerkli, K., Bjфrklund, K. R., et al., 1975. Late Quaternary sediments from Korsfjorden, western Norway. Sarsia, 58:43-66.

Bé, A. W. H., 1977. An ecological, zoogeographic and taxonomic review of Recent planktonic foraminifera. In Ramsay, A. T. S. (Ed.), Oceanic Micropaleontology: New York (Academic Press), $1: 1-100$.

Benson, R. N., 1964. Preliminary report on Radiolaria in Recent sediments of Gulf of California. In van Andel, Tj. H., and Shor, G. G. (Eds.), Marine Geology of the Gulf of California: (Mem. 3): Tulsa (American Association of Petroleum Geologists), pp. 398400 .

1966. Recent Radiolaria from the Gulf of California [Ph. D. dissert.]. University of Minnesota, Minneapolis.

Bjфrklund, K. R., 1977. Actinomma haysi, n. sp., its Holocene distribution and size variation in Atlantic Ocean sediments. Micropaleontology, 23:114-126.

Campbell, A. S., 1954. Radiolaria. In Moore, R. C. (Ed.), Treatise on Invertebrate Paleontology, Part D, Protista 3: Lawrence (Geological Society of America and University of Kansas Press), pp. 11-163.

Carnevale, P., 1908. Radiolarie e Silicoflagellati di Bergonzano (Reggio Emilia). Memorie del Reale Istituo Veneto di Scienze, Lettere ed Arti, 28(3):1-46.

Casey, R. E., 1971. Radiolarians as indicators of past and present water-masses. In Funnell, B. M., and Riedel, W. R. (Eds.), The Micropaleontology of Oceans: Cambridge (Cambridge University Press), pp. 331-341.

1977. The ecology and distribution of Recent Radiolaria. In Ramsey, A. T. S. (Ed.), Oceanic Micropaleontology: New York (Academic Press), 2:809-845.

Dinkelman, M. G., 1973. Radiolarian stratigraphy: Leg 16, Deep Sea Drilling Project. In van Andel, Tj. H., Heath, G. R., et al., Init. Repts. DSDP, 16: Washington (U.S. Govt. Printing Office), 747-813.

Dreyer, F., 1889. Morphologische Radiolarien studien. 1. Die Pylombildungen in vergleichend-anatomischer und entwicklungsgeschichtlicher Beziehung bei Radiolarien und bei Protisten uberhaupt, nebst System und Beschreibung neuer und der bis jetzt bekannten pylomatischen Spumellarien. Jena. Z. Naturwiss., 23 (n.s. Vol. 16): $1-138$.

Dumitrică, P., 1972. Cretaceous and Quaternary Radiolaria in deep sea sediments from the northeast Atlantic Ocean and Mediterranean Sea. In Ryan, W. B. F., Hsü; K. J., et al., Init. Repts. DSDP, 13: Washington (U.S. Govt. Printing Office), 829-901.

Ehrenberg, C. G., 1861. Über die Tiefgründ-Verhältnisse des Oceans am Eingange der Davisstrasse und bei Island. Kgl. Preuss. Akad. Wiss. Berlin, Monatsber., 1861:275-315.

1872a. Mikrogeologischen Studien als Zusammenfassung seiner Beobachtungen des kleinsten Lebens der Meeres-Tiefgründe aller Zonen und dessen geologischen Einfluss. Kgl. Preuss. Akad. Wiss. Berlin, Monatsber., 1872:265-322.

1872b. Mikrogeologischen Studien uber das kleinste Leben der Meeres-Tiefgründe aller Zonen und dessen geologischen Einfluss. Abh. Kgl. Akad. Wiss. Berlin, 1872:131-399.

Gartner, S., 1977. Calcareous nannofossil biostratigraphy and revised zonation of the Pleistocene. Mar. Micropaleontol., 2:1-25.

Goll, R. M., 1968. Classification and phylogeny of Cenozoic Trissocyclidae (Radiolaria) in the Pacific and Caribbbean basins, Part I. J. Paleontol., 42:1409-1432, pls. 173-176.

1969. Classification and phylogeny of Cenozoic Trissocy clidae (Radiolaria) in the Pacific and Caribbean basins, Part II. $J$. Paleontol., 43:322-339, pls. 55-60.

1972. Leg 9 synthesis, Radiolaria. In Hays, J. D., et al., Init. Repts. DSDP, 9: Washington (U.S. Govt. Printing Office), 947-1058.

1976. Morphological intergradation between modern populations of Lophospyris and Phormospyris (Trissocyclidae, Radiolaria). Micropaleontology, 22:379-418. 
Haeckel, E., 1860. Abbildungen und Diagnosen neuer Gattungen und Arten von lebenden Radiolarien des Mittelmeeres. Kgl. Preuss. Akad. Wiss. Berlin, Montasber., 1860:835-845.

1862. Die Radiolarien, Eine Monographie: Berlin (Reimer). 1887. Report on the Radiolaria collected by H.M.S. Challenger during the years 1873-1876. Rept. Voyage Challenger, 1873-1876. Zool., Vol. 18.

Hays, J. D., 1965. Radiolaria and late Tertiary and Quaternary history of Antarctic seas. Biology of Antarctic Seas II, Antarctic Research Ser., 5:125-184.

1970. Stratigraphy and evolutionary trends of Radiolaria in north Pacific deep-sea sediments. In Hays, J. D. (Ed.), Geological Investigations of the North Pacific (Mem. 126): Boulder (Geological Society of America), 185-218.

Hays, J. D., and Shackleton, N. J., 1976. Globally synchronous extinction of the radiolarian Stylatractus universus. Geology, 4:649652.

Huang, T., 1967. A new Radiolaria from the Somachi Formation, Kikai-Jima, Kagoshima Prefecture, Japan. Paleontol, Soc. Japan, Trans. N. S., no. 68:177-184, pls. 17-19.

Johnson, D. A., and Knoll, A. H., 1975. Absolute ages of Quaternary datum levels in the equatorial Pacific. Quaternary Research, 5:99-110.

Johnson, D. A., and Nigrini, C., 1980. Radiolarian biogeography in surface sediments of the western Indian Ocean, Mar. Micropaleontol., 5:111-152, pls. I-V.

Jörgensen, E., 1899. Protophyten und Protozoen im Plankton aus der norwegischen Westkuste. Bergens Museums Aarbog, for 1899, no. 6.

1905. The protist plankton and the diatoms in bottom samples. Bergens Mus. Skr., 1905:49-151, pls. 6-18.

Kling, S. A., 1971. Radiolaria: Leg 6 of the Deep Sea Drilling Project. In Fischer, A. G., Heezen, B. C., et al., Init. Repts. DSDP, 6: Washington (U.S. Govt. Printing Office), 1069-1117. 1973. Radiolaria from the eastern north Pacific, Deep Sea Drilling Project, Leg 18. In Kulm, L. D., von Huene, R., et al., Init. Repts. DSDP, 18: Washington (U.S. Govt. Printing Office), 617-671.

, 1977. Local and regional imprints on radiolarian assemblages from California coastal basin sediments. Mar. Micropaleontol., 2:207-221.

1979. Vertical distribution of polycystine radiolarians in the central north Pacific. Mar. Micropaleontol., 4:295-318.

Knoll, A. H., and Johnson, D. A., 1975. Late Pleistocene evolution of the collosphaerid radiolarian Buccinosphaera invaginata Haeckel. Micropaleontology, 21:60-68.

Ling, H.-Y., and Anikouchine, W. A., 1967. Some spumellarian Radiolaria from the Java, Philippine, and Mariana Trenches. $J$. Paleontol., 41:1481-1491, pls. 189-192.

Ling, H.-Y., Stadum, C. J., and Welch, M. L., 1971. Polycystine Radiolaria from Bering Sea surface sediments. In Farinacci, A. (Ed.), Proc. II Plankt. Conf., Roma 1970: Roma (Edizioni Technoscienza), pp. 705-729.

Molina-Cruz, A., 1977. Radiolarian assemblages and their relationship to the oceanography of the subtropical southeastern Pacific. Mar. Micropaleontol., 2:315-352.

Müller, J., 1858. Über die Thalassiocollen, Polycystinen und Acanthometren des Mittelmeeres. Abh. Kgl. Akad. Wiss. Berlin, 1858: $1-62$.

Nigrini, C., 1967. Radiolaria in pelagic sediments from the Indian and Atlantic Oceans. Bull. Scripps Inst. Oceanog., 11:1-125.
1968. Radiolaria from [the] eastern tropical Pacific sediments. Micropaleontology, 14:51-63.

1970. Radiolarian assemblages in the north Pacific and their application to a study of Quaternary sediments in core V20-130. In Hays, J. D. (Ed.), Geological Investigations of the North Pacific (Mem. 126): Boulder (Geological Society of America), 139-183.

1971. Radiolarian zones in the Quaternary of the equatorial Pacific Ocean. In Funnell, B. M., and Riedel, W. R. (Eds.), The Micropaleontology of Oceans: Cambridge (Cambridge University Press), pp. 443-461.

1977. Tropical Cenozoic Artostrobiidae (Radiolaria). Micropaleontology, 23:241-269.

Nigrini, C., and Moore, T. C., Jr., 1979. A guide to modern Radiolaria. Cushman Found. Foraminiferal Res., Spec. Publ., 16.

Petrushevskaya, M. G., and Bjørklund, K. R., 1974. Radiolarians in Holocene sediment of the Norwegian-Greenland Seas. Sarsia, 57:33-46.

Petrushevskaya, M. G., and Kozlova, G. E., 1972. Radiolaria: Leg 14, Deep Sea Drilling Project. In Hayes, D. E., Pimm, A. C., et al., Init. Repts. DSDP, 14: Washington (U.S. Govt. Printing Office), 495-648.

Popofsky, A., 1908. Die Radiolarien der Antarktis (mit Ausnahme der Tripyleen). Deutsche Südpolar-Expedition, 1901-1903, 10 (Zool., Vol. 2) (3):183-305, pls. 20-36.

1912. Die Sphaerellarien des Warmwassergebietes. Deutsche Südpolar-Expedition, 1901-1903, 13 (Zool., Vol. 5) (2):73-159. 1913. Die Nassellarien des Warmwassergebietes. Deutsche Südpolar-Expedition, 1901-1903, 14 (Zool., Vol. 6):217-416, pls. 28-38.

Renz, G. W., 1974. Radiolaria from Leg 27 of the Deep Sea Drilling Project. In Veevers, J. J., Heirtzler, J. R., et al., Init. Repts. DSDP, 27: Washington (U.S. Govt. Printing Office), 769-841.

1976. The distribution and ecology of Radiolaria in the central Pacific: plankton and surface sediments. Bull. Scripps Inst. Oceanog., 22:267.

Riedel, W. R., 1957. Radiolaria: a preliminary stratigraphy. Rept. Swedish Deep-Sea Expedition, Ser. B., 6 (Pt. 10):217-255.

1958. Radiolaria in Antarctic sediments. B.A.N.Z. Antarctic Research Expedition Reports, Ser. B., 6(Pt. 10):217-255.

Riedel, W. R., and Sanfilippo, A., 1971. Cenozoic Radiolaria from the western tropical Pacific, Leg 7. In Winterer, E. L., Riedel, W. R., et al., Init. Repts. DSDP, 7, Pt. 2: Washington (U.S. Govt. Printing Office), 1529-1672.

1977. Cainozoic Radiolaria. In Ramsay, A. T. S. (Ed.), Oceanic Micropalaeontology: New York (Academic Press), 2: 847-912.

1978. Stratigraphy and evolution of tropical Cenozoic radiolarians. Micropaleontology, 24:61-96.

Stöhr, E., 1880. Die Radiolarienfauna der Tripoli von Grotte, Provinz Girgenti in Sicilien. Palaeontographica, 26 (Ser. 3, Vol. 2): 69-124 (and Corrigenda), pls. 17-23.

van Andel, Tj. H., 1964. Recent marine sediments of Gulf of California. In van Andel, Tj. H., and Shor, G. G. (Eds.), Marine Geology of the Gulf of California (Mem. 3): Tulsa (American Association of Petroleum Geologists), 216-310.

Vinassa de Regny, P., 1900. Radiolari Miocenici Italiani. Memorie della R. Accademia delle Scienze dell'Istituto di Bologna, Ser. 5, 8: 227-257. 


\section{R. N. BENSON}

\section{APPENDIX}

Radiolarian Names

Acanthocorys variabilis

Acanthosphaera elliptica

Acrobotrissa cribosa (Plate 9, Fig. 5)

Acrobotyrs disolenia

A. sp. cf. A. disolenia (Plate 9, Fig. 6-7)

Acrosphaera murrayana

Actinomma antarctium

A. arcadophorum

A. haysi

A. hystrix

A. leptodermum

A. medianum

A. sp.

Actinosphaera cristata

Amphicraspedum wyvilleanum

Amphiplecta acrostoma

A. cylindrocephala (Plate 8, Fig. 5)

Amphirhopalum

A. virchowii (Plate 2, Figs. 1-3)

A. ypsilon (Plate 2, Figs. 4-7)

Amphisphaera cristata (Plate 4, Fig. 5)

A. cronos

A. pluto

A. uranus

Amphispyris costata

A. costata-thorax

A. reticulata

A. subquadrata

A. toxarium

A. zonarius

Amphitholus acanthometra

Anomalacantha dentata

Anthocyrtidium angulare (Plate 3, Fig. 1)

A. cineraria

A. ophirense

A. sp. cf. A. angulare (Plate 3, Figs. 2-3)

A. zanguebaricum

A. oxycephalis

Arachnocorys pentacantha

A. umbellifera (Plate 8, Fig. 6)

Artopilium elegans

A. undulatum

Axoprunum angelinum (Plate 1, Figs. 1-4)

Bathropyramis woodringi

Botryocyrtis caput-serpentis

B. quinaria

B. scutum

B. sp.

Botryopyle sp.

Botryostrobus aquilonaris

B. auritus-australis

Buccinosphaera invaginata

Callimitra emmae

C. sp.

Calocyclas amicae

C. monumentum

Carpocanarium papillosum

C. spp.

Carpocanistrum sp. A

C. spp.

C.(?) sp.

Carpocanium petalospyris

C. spp.

Carposphaera acanthophora

Cenosphaera coronata

C. cristata

C. perforata

C. spp.

C.(?) sp. aff. C. perforata (Plate 4, Fig 4)

Ceratospyris borealis

C. pentagona

C. polygona
Choenicosphaera murrayana

Cinclopyramis infundibulum

Circodiscus microporus

Cladococcus abietinus

C. cervicornis (Plate 4, Fig. 1)

C. scoparius

C. stalactites (Plate 4, Fig. 2)

Cladoscenium tricolpum

Clathrocanium coronatum

C. ornatum

C. sp.

Clathrocircus stapedius (Plate 7, Figs. 5-7)

Clathrocorys murrayi

C. sp.

Clathrocyclas? sp.

Clathrocycloma davisiana

Clathromitra pterophormis (P1. 9, Fig. 8)

Collosphaera sp. A

C. tuberosa (Plate 1, Figs. 5-6)

$C$.(?) sp. (Plate 4, Fig. 3)

Conarachnium sp.

Cornutella profunda

Corocalyptra

C. cervus

C. killmari

C. kruegeri

Cubotholus octoceras

C. regularis

Cycladophora davisiana

Cypassis irregularis

Cyrtopera laguncula

Dendrospyris binapteronis

D. damaecornis

Desmospyris anthocyrtoides

Dictyocephalus mediterraneus

D. papillosus

Dictyoceras acanthicum

D. pyramidale

Dictyocoryne profunda

D. sp. (Plate 6, Fig. 2)

D. truncatum (Plate 6, Fig. 1)

Dictyocryphalus papillosus

Dictyophimus crisiae

D. gracilipes

D. infabricatus

D. platycephalus (Plate 8, Fig. 7)

D. sp. cf. D. tripus (Plate 8, Fig. 4)

D. tetracanthus

D. tripus

Diploplegma banzare

Discopyle(?) sp.

Disolenia quadrata

D. variabilis

Doryconthidium hexactis

D. (?) sp.

Druppatractus acquilonius

D. irregularis

D. pyriformis

D. variabilis

D. (?) sp.

Echinomma delicatulum

E. delicatum

E. leptodermum

Elaphococcus cervicornis

Eucecryphalus cervus

E.(?) sp. (Plate 9, Fig. 4)

Euchitonia echinata

E. elegans

E. furcata

E. mulleri

E. sp.

E. sp. cf. E. triangulum (Plate 5, Figs. 4-5)

E. triangulum

Eucoronis nephrospyris

$E$.(?) sp. 
Eucyrtidium

E. anomalum

E. calvertense

E. hertwigii

E. hexagontum

E. hexastichum

E. infundibulum

E. matuyamai

E. papillosum

E. (?) hexastichum (Plate 9, Figs. 9-11)

Eusyringium siphonostoma

Giraffospyris angulata

Haliomma erinaceum

Heliodiscus asteriscus

H. echiniscus

Heliosphaera radiata

Helotholus histricosa (Plate 8, Fig. 1-3)

Heteracantha dentata

Hexacontium arachnoidale

H. enthacanthum

H. heracliti

$H$. heteracantha

H. laevigatum

$H$. sp. cf. H. heracliti (Plate 4, Fig. 7)

Hexalonche anaximandri

$H$. heracliti

H. heteracantha

Hexapyle dodecantha (Plate 6, Figs. 6-7)

$H$. spp.

Hexastylus triaxonius (Plate 4, Fig. 6)

Hymeniastrum euclidis

H. koellikeri

Lamprocyclas junonis

L. maritalis

L. maritalis maritalis

L. maritalis polypora

L. maritalis ventricosa

Lamprocyrtis haysi

L. neoheteroporos (Plate 3, Figs. 4-6)

L. nigriniae

L.(?) hannai

Lampromitra coronato

L. quadricuspis (Plate 8, Fig. 8)

Larcopyle butschlii

L.(?) sp.

Larcospira quadrangula

Lipmanella dictyoceras

L. irregularis

L. tribranchiata (Plate 9, Fig. 1)

L.(?) dogieli

Liriospyris reticulata

L. sp.

L. sp. 2

L. toxarium

L.(?) toxarium A

Litharachnium tentorium

Lithelius minor

L. (?) sp.

Lithocampe anomala

Lithomelissa galeata

L. hystrix

L. laticeps (Plate 9, Fig. 3)

L. monoceras

L. spp.

L. thoracites (Plate 9, Fig. 2)

Lithomitra infundibulum

L. lineata

Lithopera bacca

Lithopilium sphaerocephalum

Lithostrobus hexagonalis

$L$. hexastichus

Lophocorys polyacantha

Lophophaena capito

L. cylindrica

Lophophaenoma witjazii
Lophospyris pentagona hyperborea

L. pentagona pentagona

L. pentagona quadriforis

Lychnodictyum challengeri

Neosemantis distephanus

Nephrodictyum renilla

Nephrospyris renilla

Obeliscus pseudocuboides

Octopyle stenozona

Ommatartus tetrathalamus

Ommatodiscus pantanellii

$O$. sp.

Panartus tetrathalamus

$P$. teirathalamus tetrathalamus

Patagospyris(?) sp.

Peridium longispinum

$P$. sp.

$P$. spinipes

Peripyramis circumtexto

Petalospyris ophirensis

Phormacantha hystrix (Plate 7, Fig. 12)

Phormocyrtis fastuosa

Phormospyris scaphipes

P. stabilis capoi

P. stabilis scaphipes

P. stabilis stabilis

P. tricostata

Phormostichoartus corbula

Phorticium pylonium (Plate 7, Figs. 15-16)

Plagiacantha(?) panarium (Plate 7, Figs. 10-11)

Plagonium sp. cf. P. sphaerozoum (Plate 7, Figs. 1-2)

$P$. sphaerozoum

Plectacantha oikiskos (Plate 7, Figs. 13-14)

$P$.(?) sp.

Plectophora triacantha

Plectopyramis dodecomma

Polysolenia murrayana

P.(?) sp.

Porodiscus microporus

P.(?) sp. B

Prunopyle antarctica

Pseudocubus obeliscus (Pl. 7, Figs. 8-9)

Pseudodictyophimus gracilipes

Psilomelissa calvata

$P$. galeata

Pterocanium bicorne

$P$. elegans

$P$. grandiporus

P. korotnevi

$P$. praetextum

P. praetextum eucolpum

P. praetextum praetextum

P. prismatium

P. prosperinae

P. sp.

P. trilobum

Pterocorys

P. clausus

P. columba

P. hertwigii

$P$. hirundo

P. killmari

P. minythorax

P.(?) sp.

$P$. zancleus

Pylonium sp. (Plate 6, Fig. 5)

Rhodospyris $\mathrm{sp}$.

Saturnalis circularis

Semantis distephanus

Sethoconus(?) dogieli

Sethophormis pentalactis

Siphocampe arachnea

$S$. corbula

S. lineata

S. nodosaria 


\section{R. N. BENSON}

S. sp.

Siphocampium cornutella

S. cylindrica

S. erucosum

S. polyzona

S. seriatus

$S$. sp.

Siphonosphaera polysiphonia

S. socialis

Sphaeropyle langii

Spirema sp. (Plate 6, Figs. 3-4)

Spirocyrtis gyroscalaris

S. scalaris

Spongaster disymmetricus

$S$. tetras

S. tetras tetras

Spongocore diplocylindrica

S. puella

Spongodiscus biconcavus

S. sp. 3

Spongoliva ellipsoides

Spongopyle osculosa

Spongosphaera streptacantha

Spongotrochus glacialis

S. sp. cf. S. glacialis (Plate 5, Fig. 3)

Spongurus elliptica

S. sp.

Stichopera pectinata

Stichopilium bicorne

$S$. annulatum

S. anocor

Stichopterygium anomalum

Stylacontarium acquilonium

S. bispiculum (Plate 1, Figs. 7-10)

Stylatractus spp.

S. universus
Stylochlamydium asteriscus

S. venustum

Stylodictya multispina

S. validispina

Stylosphaera lithatractus

Tessarastrum straussi

Tetrapyle octacantha

Thecosphaera spp.

Theocalyptra davisiana

T. davisiana cornutoides

T. davisiana davisiana

T. sp

Theoconus hertwigii

T. minythorax

T. zancleus

Theocorys veneris

Theocorythium trachelium trachelium

$T$. vetulum (Plate 3, Figs. 7-9)

Theopilium triscostatum

Tholospyris devexa

T. kantiana

T. procera

T. scaphipes

Trematodiscus microporus

Triceraspyris damaecornis

Tricolospyris kantiana

Tristylospyris scaphipes

Verticillata hexacantha

Xiphatractus circularis

$X$. cronos (Plate 4, Fig. 8)

$X$. pluto (Plate 5, Figs. 1-2)

Xiphospira circularis

Zygocampe chrysalidium

Zygocircus capulosus

Z. productus

Z. sp. (Plate 7, Figs. 3-4) 

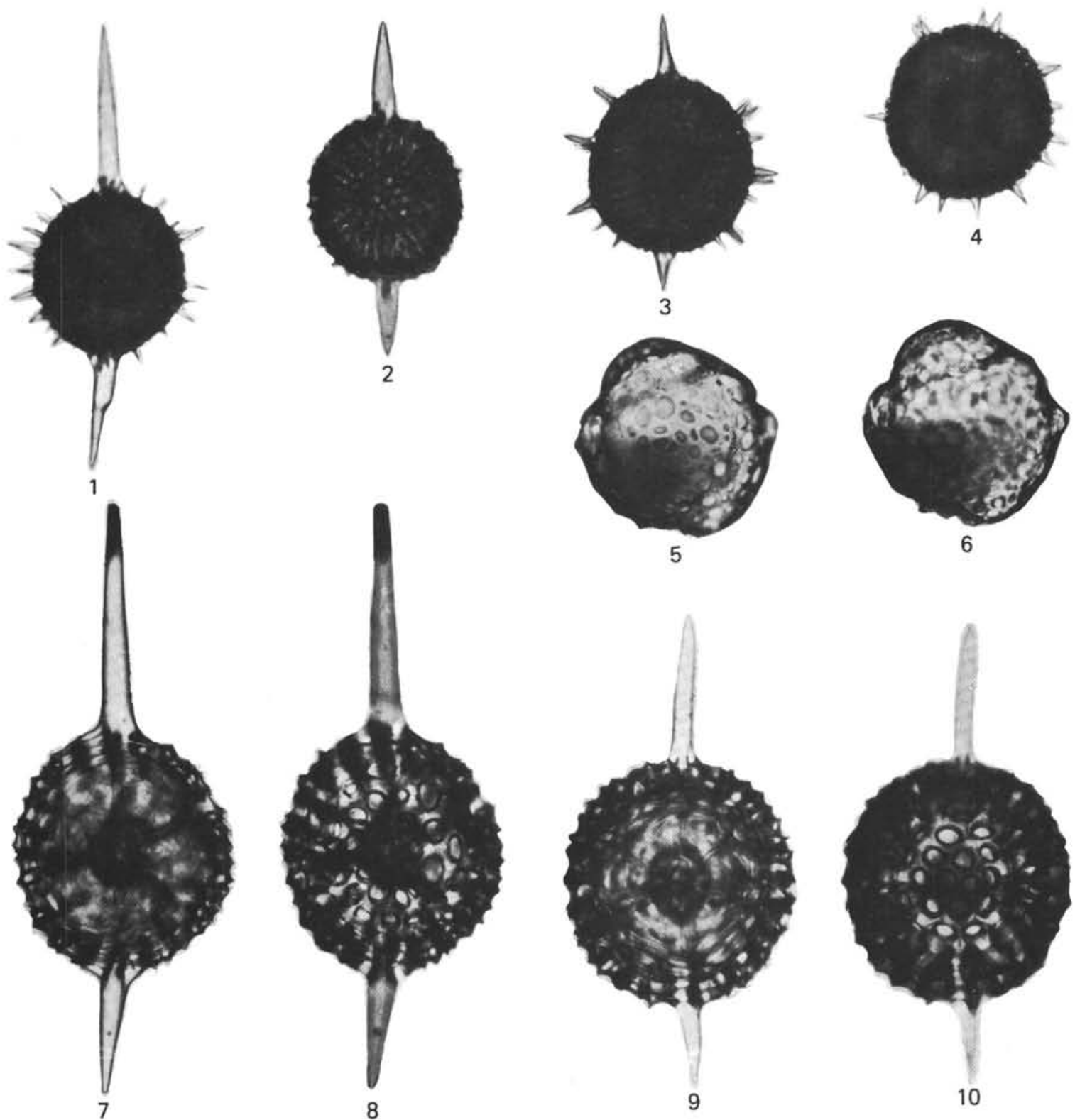

4

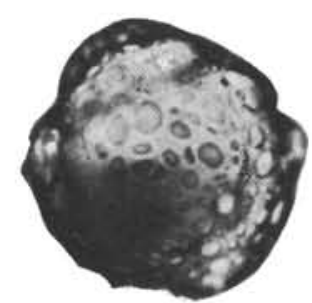

5

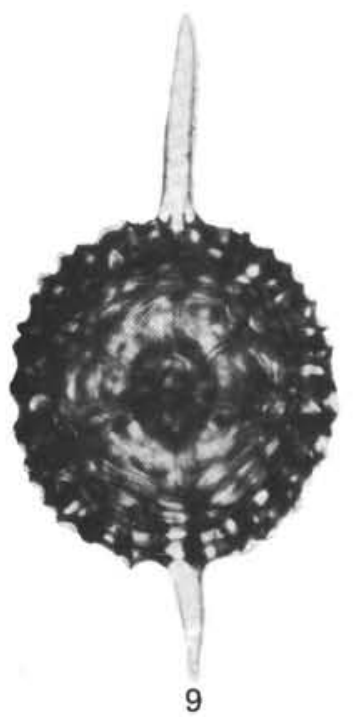

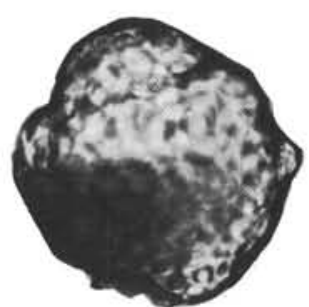

6

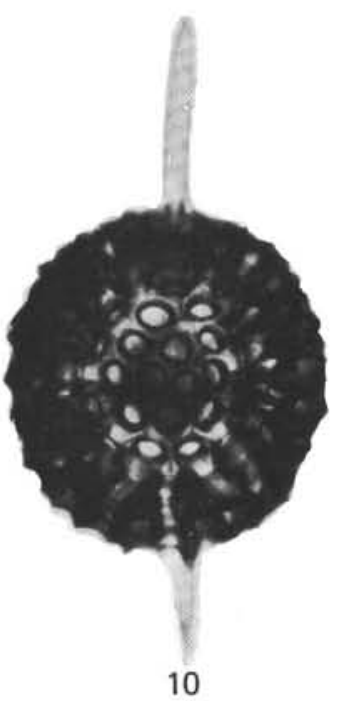

$100 \mu \mathrm{m}$

Plate 1. Radiolarians of Quaternary age from Leg 65 samples. (Scale bar equals $100 \mu \mathrm{m}$.) 1-4. Axoprunum angelinum (Campbell and Clark). 1. Sample 483-6,CC (16-18 cm), E43/2. 2. Sample 483-9,CC (10-12 cm), slide a, V13/4. 3, 4. Tests with polar spines reduced or lacking, typical of species at highest occurrence in Leg 65 samples, (3) Sample 484A-5, CC (5-7 cm), J20/2, (4) Sample 484A-5, CC (5-7 cm), X7/2. 5, 6. Collosphaera tuberosa Haeckel. Sample 483-5, CC (5-7 cm), slide a, Z24/4, (5) focus on upper surface, (6) focus on periphery. 7-10. Stylacontarium bispiculum Popofsky. Tests closely resembling Stylacontarium acquilonium (= Druppatractus acquilonius Hays) but having a spherical outer medullary shell rather than an ellipitical shell protruding at the connecting bars, $(7,8)$ Sample $483-9, C C(10-11 \mathrm{~cm})$, slide a, X12/1, focus on medullary shell and on surface of cortical shell, respectively, $(9,10)$ Sample 482A-1,CC, H26/0, focus on medullary shell and on surface of cortical shell, respectively. Note: Species shown in Plates 1-3 are either biostratigraphic marker species, are related to these, or have potential utility in Quaternary radiolarian biostratigraphy. (Slide numbers are followed by England Finder coordinates.) 


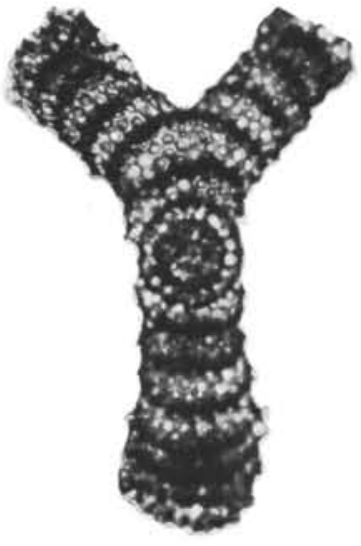

1

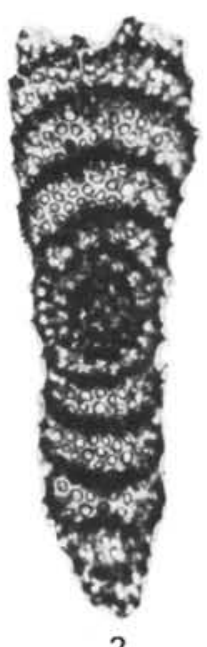

2

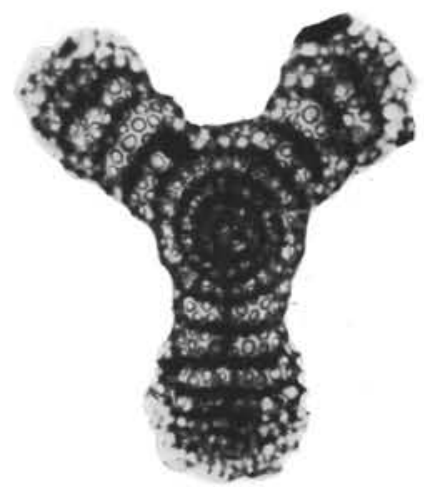

3

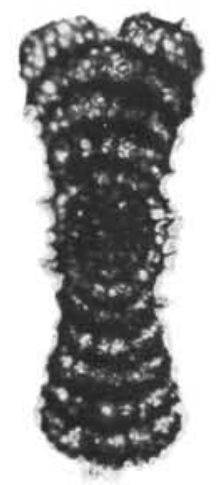

4

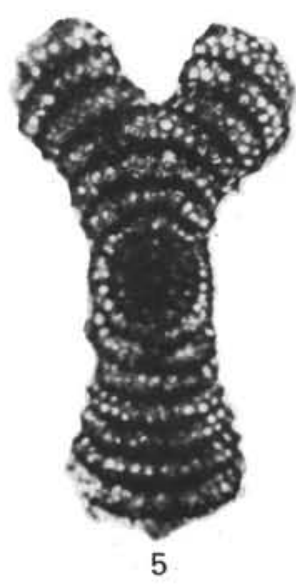

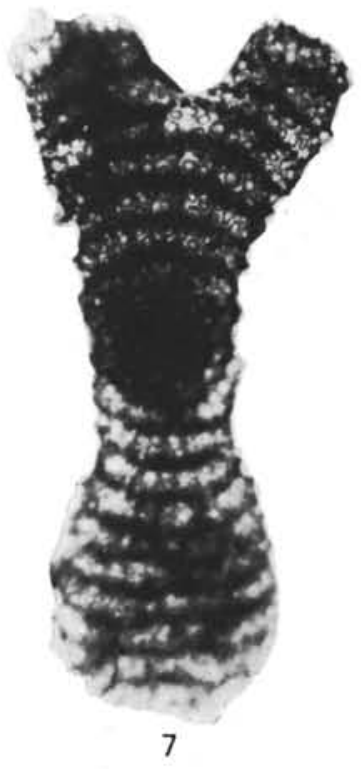

$100 \mu \mathrm{m}$

Plate 2. Radiolarians of Quaternary age from Leg 65 samples. (Scale bar equals $100 \mu \mathrm{m}$.) 1-3. Amphirhopalum virchowii (Haeckel), (1) Sample 483-6,CC (16-18 cm), R18/0, (2) Sample 482B-3,CC (5-7 cm), Y26/0, (3) Sample 485A-3,CC (2-4 cm), O35/1. 4-6. Amphirhopalum ypsilon Haeckel. Low occurrence forms showing three chambers on the forked arm before bifurcation, (4) Sample $482 \mathrm{~B}-2, \mathrm{CC}(4-6 \mathrm{~cm}), \mathrm{R} 7 / 3(60.73 \mathrm{~m}$ sub-bottom), (5) Sample 485A-3,CC (2-4 cm), G17/0 (73.22 m sub-bottom). (6) Sample 485A-3,CC (2-4 cm), N19/4 (73.22 m sub-bottom). 7. Amphirhopalum ypsilon Haeckel. High occurrence form showing five chambers on the forked arm before bifurcation, Sample 482A-1,CC, $\mathrm{H} 26 / 0$ (4.00 $\mathrm{m}$ sub-bottom).

See Note, Plate 1. (Slide numbers are followed by England Finder coordinates.) 

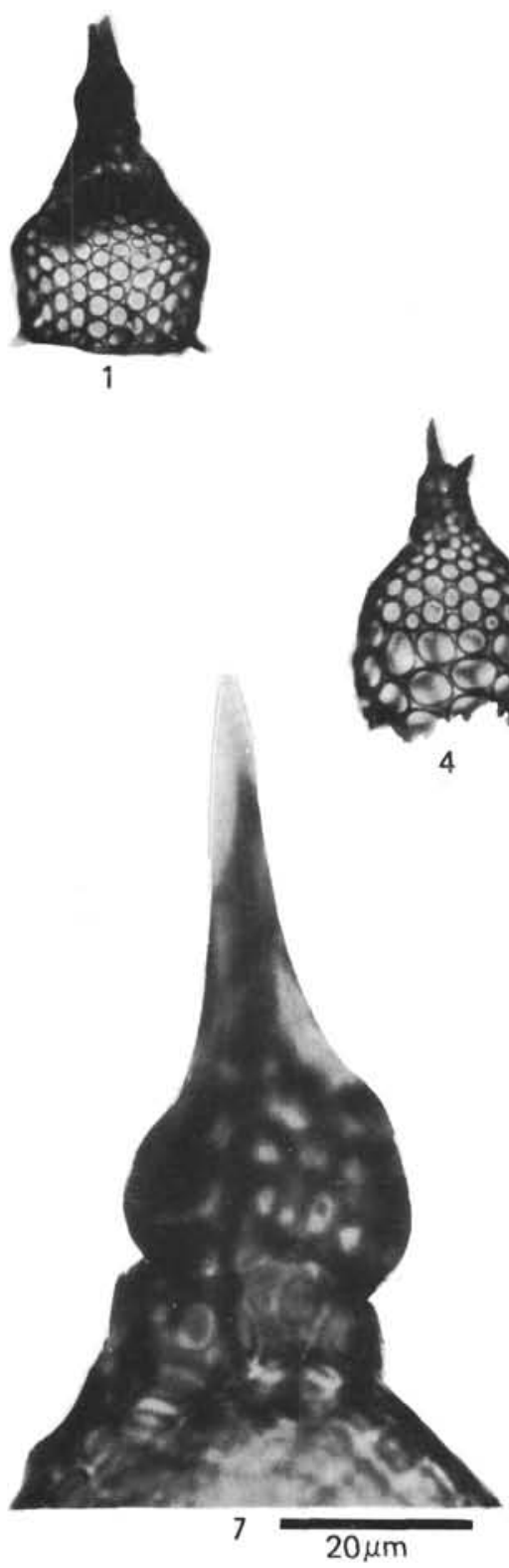

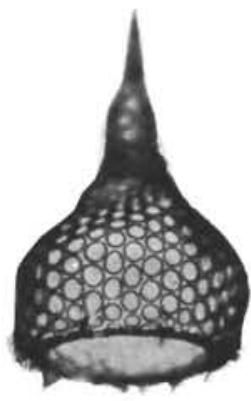

2

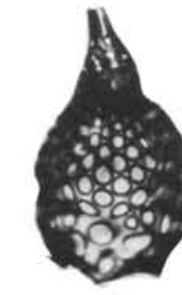

5

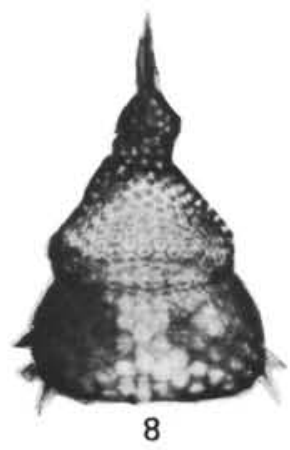

$100 \mu \mathrm{m}$

Figs. 1-6,8
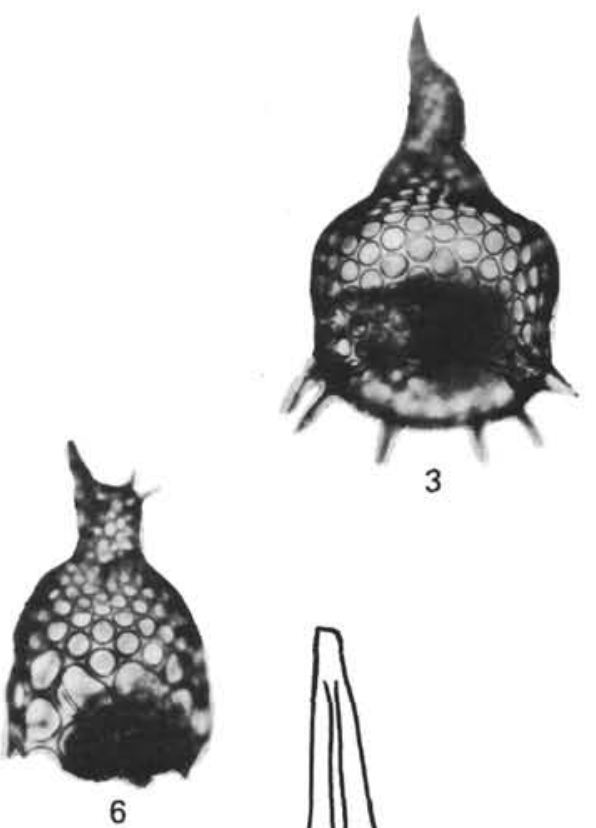

3

Plate 3. Radiolarians of Quaternary age from Leg 65 samples. (Scale bars equal $100 \mu \mathrm{m}$ for Figs. 1-6, 8 and $20 \mu \mathrm{m}$ for Figs. 7, 9.) 1. Anthocyrtidium angulare Haeckel. Sample 483-9-1, 74-76 cm, slide a, D17/2. 2-3. Anthocyrtidium sp. cf. A. angulare Haeckel, (2) Sample 483-9-1, 74-76 cm, slide b, O44/0, (3) Sample 483-9-1, 74-76 cm, slide b, M34/0. 4-6. Lamprocyrtis neoheteroporos Kling, (4) Sample 483-8, CC (11-13 $\mathrm{cm}), \mathrm{D} 40 / 1$, (5) Sample 485A-3,CC (2-4 cm), T5/0, (6) Sample 483-8,CC (11-13 cm), E36/0. 7-9. Theocorythium vetulum Nigrini, all of same specimen, Sample 483-8,CC (11-13 cm), E32/0. Figures 7 and 9 illustrate the paired cephalic lobes directly beneath the larger unpaired lobe, a characteristic of the genus Theocorythium.

See Note, Plate 1. (Slide numbers are followed by England Finder coordinates.) 


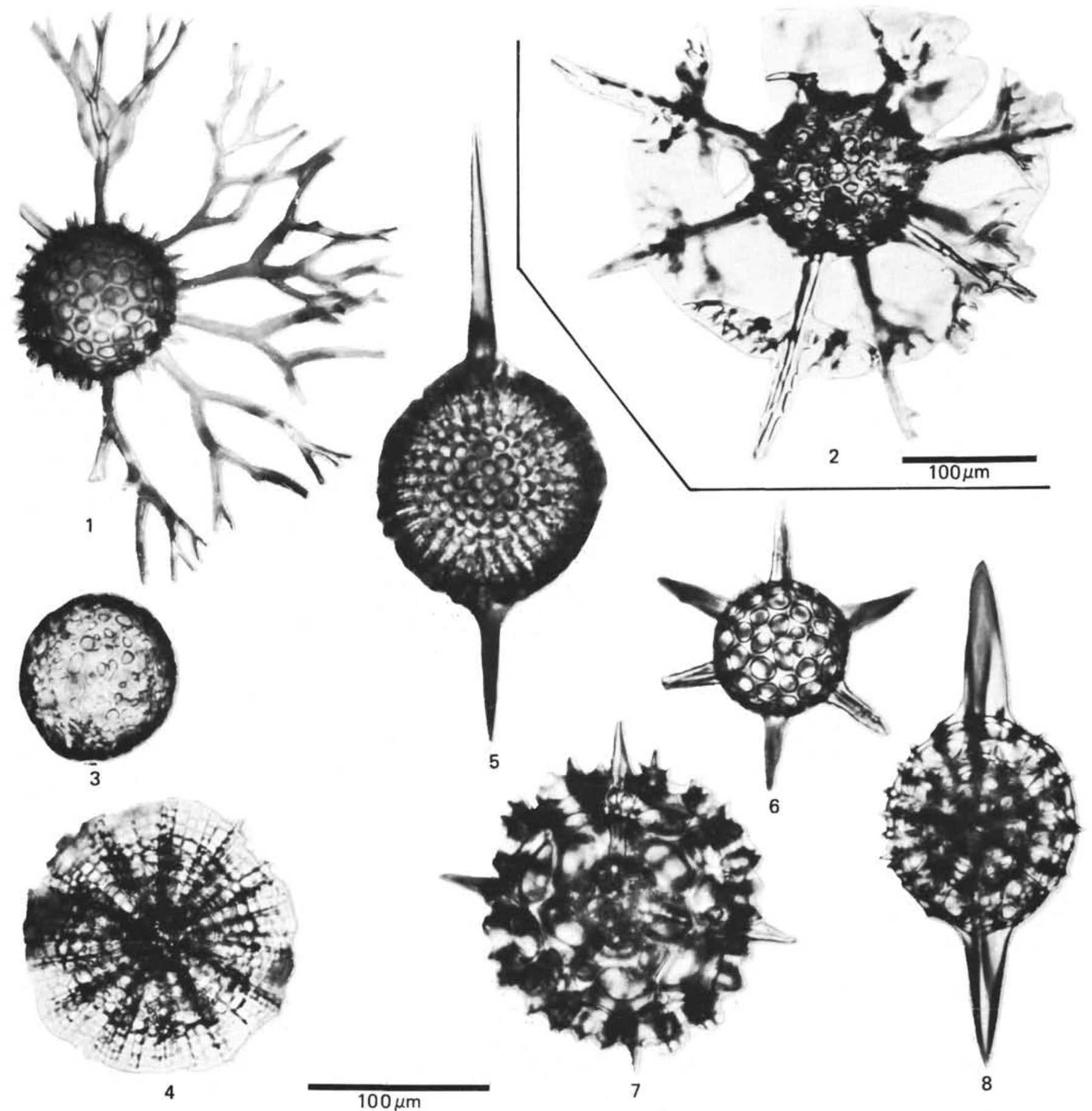

$100 \mu \mathrm{m}$

Plate 4. Radiolarians from Holocene sediments in the Gulf of California. (Scale bars equal $100 \mu \mathrm{m}$.) 1. Cladococcus cervicornis Haeckel. VS-R133b, 1-3 cm, V17/2. Benson, 1966, pl. 6, fig. 1. 2. Cladococcus stalactities Haeckel. VS-R-136a, 1-3 cm, M6/3. Benson, 1966, pl. 6, fig. 2. 3. Collosphaera(?) sp. VS-R-60a, 3-5 cm, K12/1, Benson, 1966, pl. 2, fig. 1. 4. Cenosphaera(?) sp. aff. C. perforata Haeckel. VS-R-60a, 3-5 $\mathrm{cm}, \mathrm{S} 15 / 4$. Benson, 1966, pl. 2, fig. 6. 5. Amphisphaera cristata Carnevale. VS-R-46a, 1-3 cm, M32/1. Benson, 1966, pl. 3, fig. 5. 6. Hexastylus triaxonius Haeckel. VS-R-71a, 1-3 cm, E32/3. Benson, 1966, pl. 3, fig. 6. 7. Hexacontium sp. cf. H. heracliti (Haeckel). VS-R-71a, 1-3 $\mathrm{cm}, \mathrm{J6} / 0$. Benson, 1966, pl. 4, fig. 8. 8. Xiphatractus cronos (Haeckel). VS-R-56a, 1-3 cm, J55/1. Benson, 1966, pl. 7, fig. 12. Note: Plates 4 through 9 illustrate species from Holocene sediments in the Gulf of California that are part of the Quaternary-age assemblages from Leg 65 and that have not been illustrated previously by Benson (1964) or other contemporary radiolarian workers. The photographs are from Benson's (1966) plates. (Slide numbers are followed by England Finder coordinates. The numbers preceded by VS-R- refer to sampling stations in the Gulf of California [this chapter, Fig. 2].) 

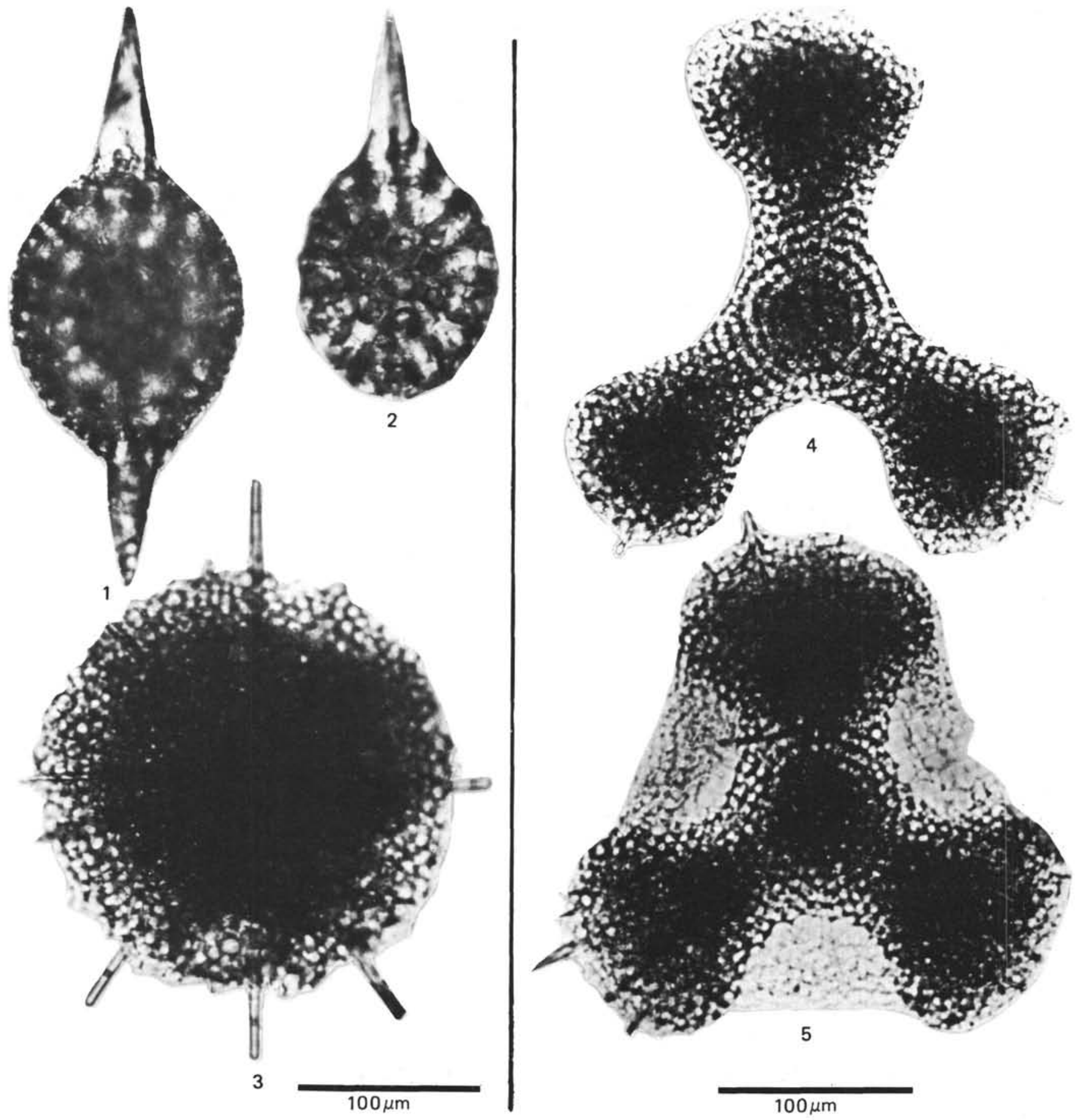

Plate 5. Radiolarians from Holocene sediments in the Gulf of California. (Scale bars equal $100 \mu \mathrm{m}$.) 1-2. Xiphatractus pluto (Haeckel), (1) VS-R27b, 1-3 cm, D15/0. Benson, 1966, pl. 7, fig. 17, (2) VS-R-27b, 1-3 cm, D15/0. Focus on surface of cortical shell. Benson, 1966, pl. 7, fig. 16. 3. Spongotrochus sp. cf. S. glacialis Popofsky. VS-R-81a, 1-3 cm, S41/2. Benson, 1966, pl. 11, fig. 4. 4-5. Euchitonia sp. cf. E. triangulum (Ehrenberg), (4) VS-R-71a, 1-3 cm, J38/0. Benson, 1966, pl. 13, fig. 1, (5) VS-R-34a, 3-5 cm, S30/3. Benson, 1966, pl. 13, fig. 3. See Note, Plate 4. 

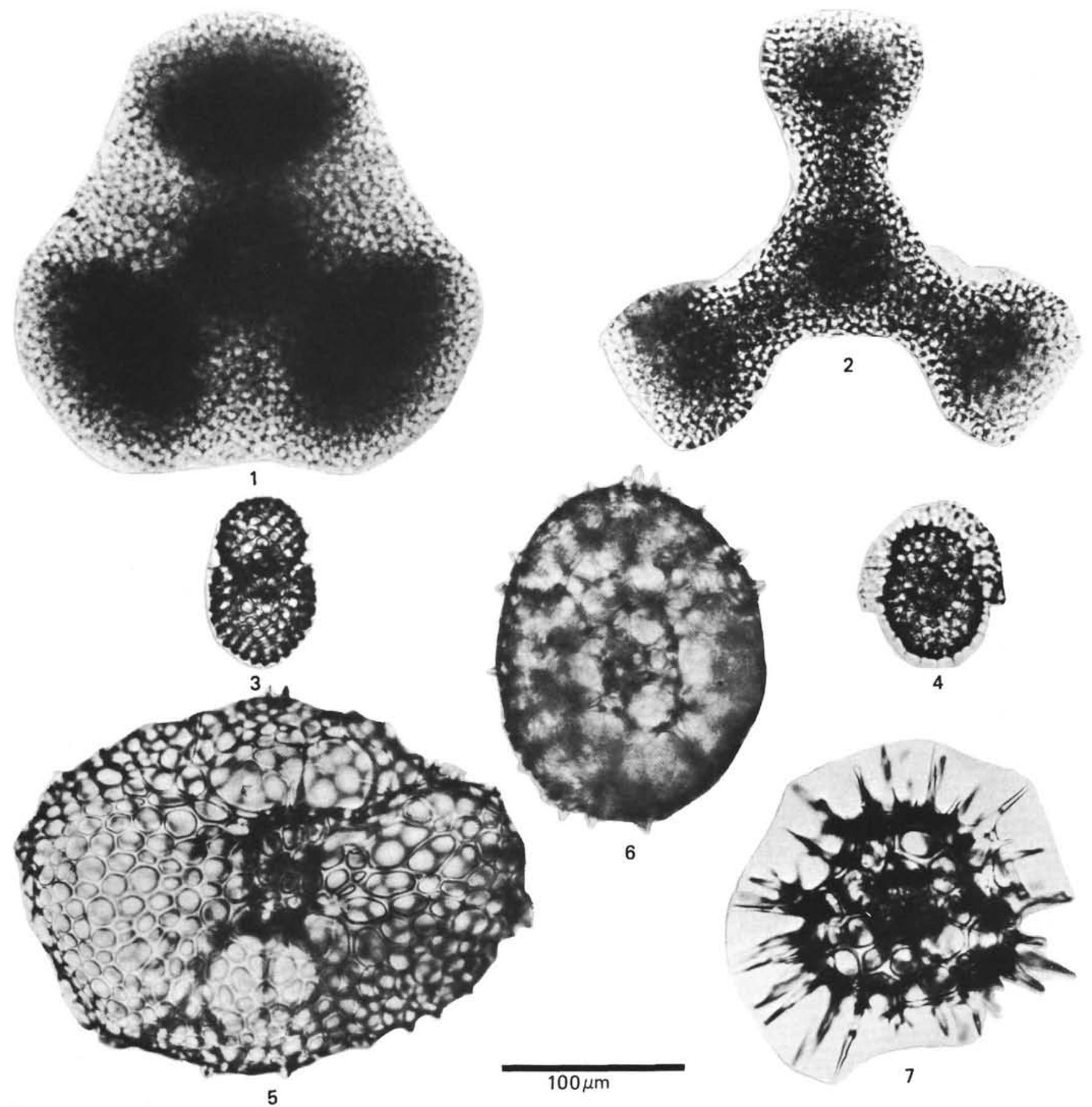

Plate 6. Radiolarians from Holocene sediments in the Gulf of California. (Scale bar equals $100 \mu \mathrm{m}$.) 1. Dictyocoryne truncatum (Ehrenberg). VSR-56a, 1-3 cm, Q20/0. Benson, 1966, pl. 15, fig. 1. 2. Dictyocoryne sp. VS-R-27b, 1-3 cm, Z52/4. Benson, 1966, pl. 12, fig. 4. 3-4. Spirema sp., (3) VS-R-27b, 1-3 cm, K19/2. Benson, 1966, pl. 18, fig. 9, (4) VS-R-27b, 1-3 cm, R50/2. Benson, 1966, pl. 18, fig. 10. 5 Pylonium sp. VSR-81a, 1-3 cm, X38/4. Benson, 1966, pl. 16, fig. 2. 6-7. Hexapyle dodecantha Haeckel group, (6) VS-R-184b, 1-3 cm, Y17/4. Benson, 1966, pl. 18, fig. 13, (7) VS-R-81a, $1-3 \mathrm{~cm}$, Y51/2. Benson, 1966, pl. 18, fig. 15. See Note, Plate 4. 

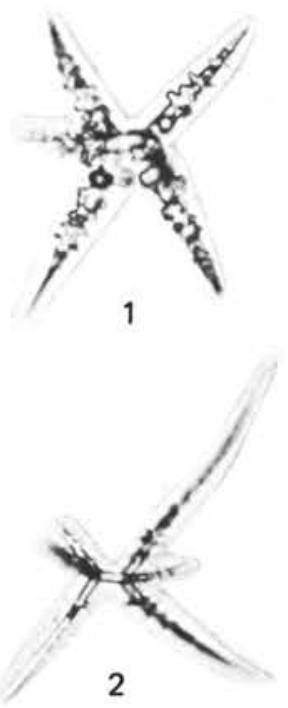

2

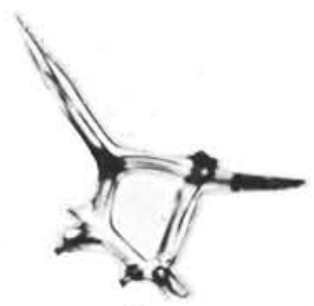

3

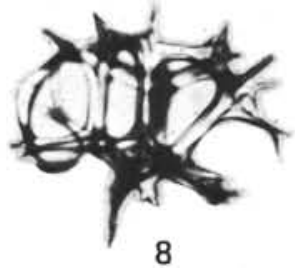

8

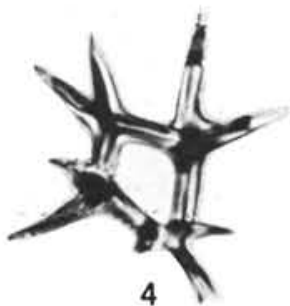

4

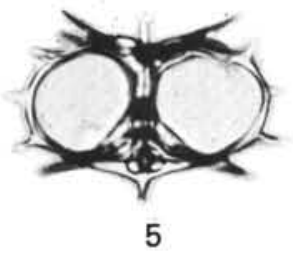

,

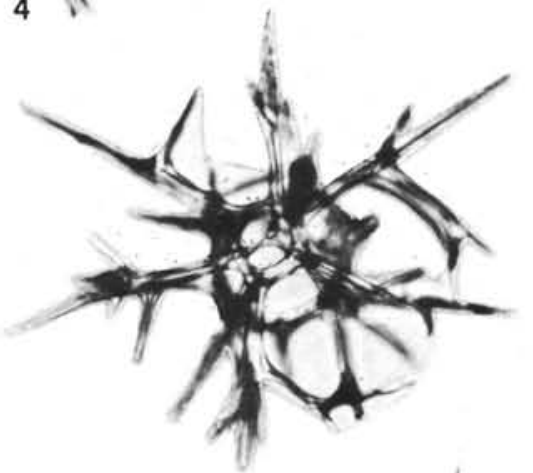

9

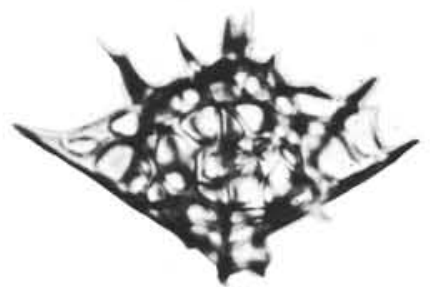

10

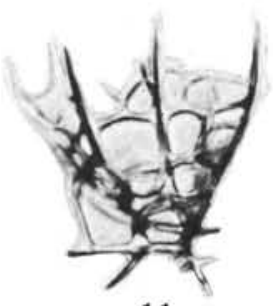

11

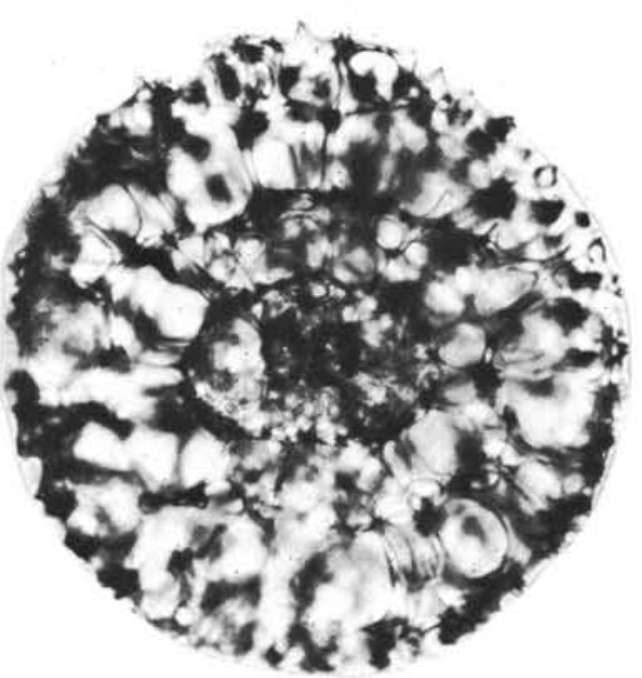

15

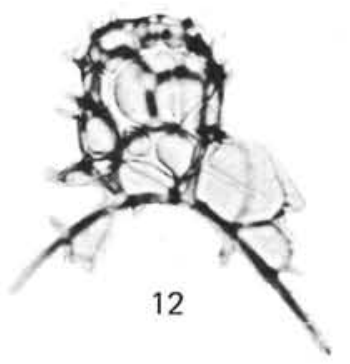

$100 \mu \mathrm{m}$
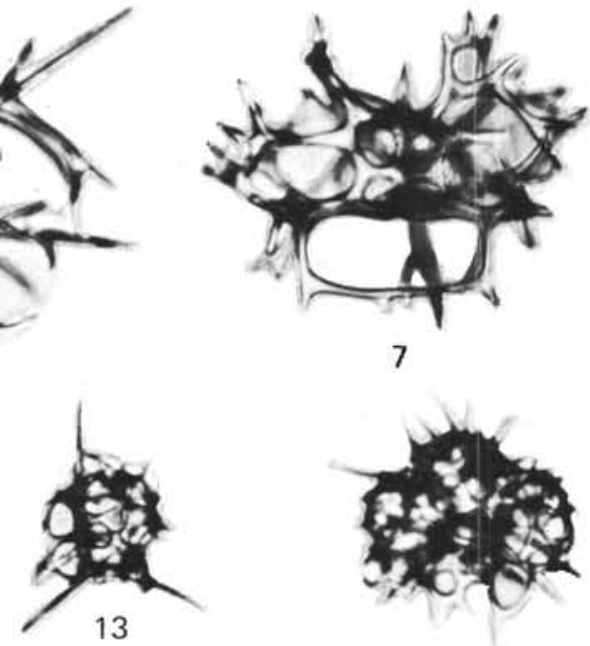

7

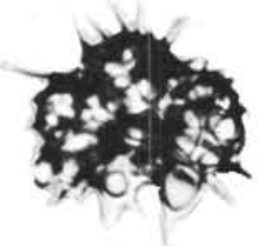

14

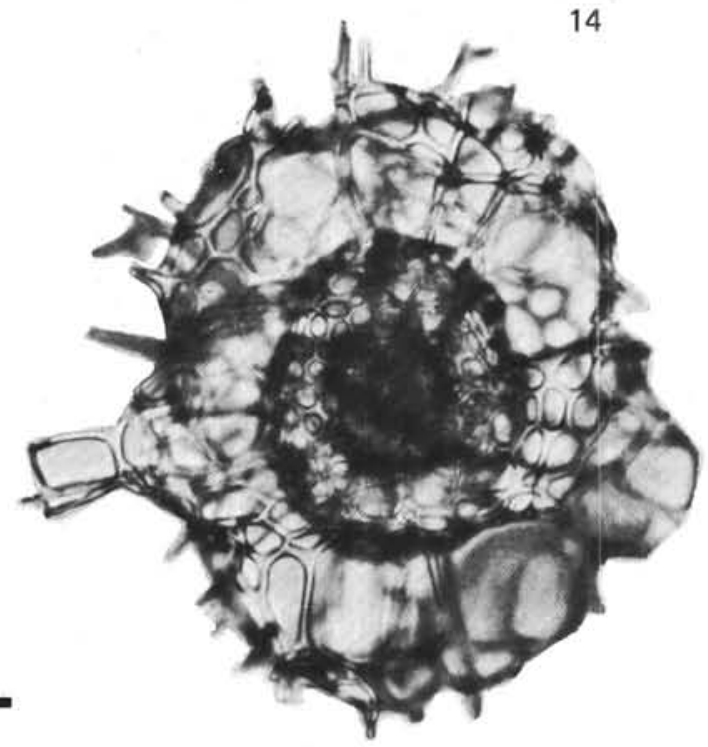

16

Plate 7. Radiolarians from Holocene sediments in the Gulf of California. (Scale bar equals $100 \mu \mathrm{m}$.) 1-2. Plagonium sp. cf. P. sphaerozoum Haeckel, (1) VS-R-151a, 1-3 cm, C44/0. Benson, 1966, pl. 19, fig. 13, (2) VS-R-93b, 1-3 cm, U11/4. Benson, 1966, pl. 19, fig. 12. 3-4. Zygocircus sp., (3) VS-R-93b, 1-3 cm, X17/4. Benson, 1966, pl. 19, fig. 16, (4) VS-R-133b, 1-3 cm, O36/0. Benson, 1966, pl. 19, fig. 17. 5-7. Clathrocircus stapedius Haeckel, (5) VS-R-81a, 1-3 cm, G20/1. Ventral view. Benson, 1966, pl. 21, fig. 11, (6) VS-R-81a, 1-3 cm, G19/4. Basal view showing collar pores. Benson, 1966, pl. 21, fig. 12, (7) VS-R-133b, 1-3 cm, E30/3. Apical view. Benson, 1966, pl. 21, fig. 13. 8-9. Pseudocubus obeliscus Haeckel, (8) VS-R-133b, 1-3 cm, D28/1. Left lateral view. Benson, 1966, pl. 22, fig. 6, (9) VS-R-133b, 1-3 cm, F43/0. Basal view, focus on collar ring. Benson, 1966, pl. 22, fig. 4. 10-11. Plagiacantha(?) panarium Dumitrica, (10) VS-R-81a, 1-3 cm, U34/0. Benson, 1966, pl. 23, fig. 21, (11) VS-R-151a, 1-3 cm, N16/0. Left lateral view. Benson, 1966, pl. 23, fig. 23. 12. Phormacantha hystrix Jorgensen. VS-R-81a, 1-3 cm, J52/4. Left lateral view. Benson, 1966, pl. 23, fig. 26. 13-14. Plectacantha oikiskos Jorgensen, (13) VS-R-191a, 1-3 cm, H20/3. Right lateral view. Benson, 1966, pl. 23, fig. 19, (14) VS-R-133b, 1-3 cm, M41/0. Right lateral view. Benson, 1966, pl. 23, fig. 20. 15-16. Phorticium pylonium Haeckel group, (15) VS-R-93b, 1-3 cm, Y22/1. Polar view. Benson, 1966, pl. 17, fig. 3, (16) VS-R-81a, 1-3 cm, K38/4. Frontal view. Benson, 1966, pl. 16, fig. 9.

See Note, Plate 4. 

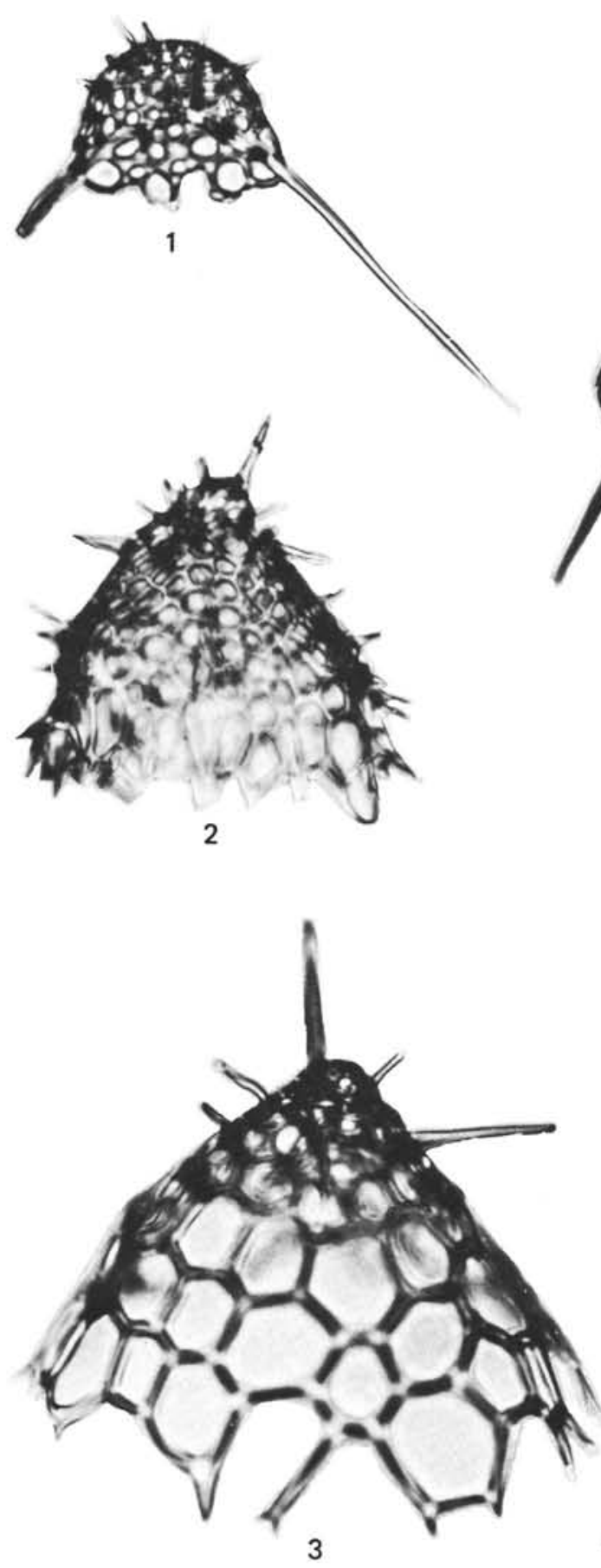

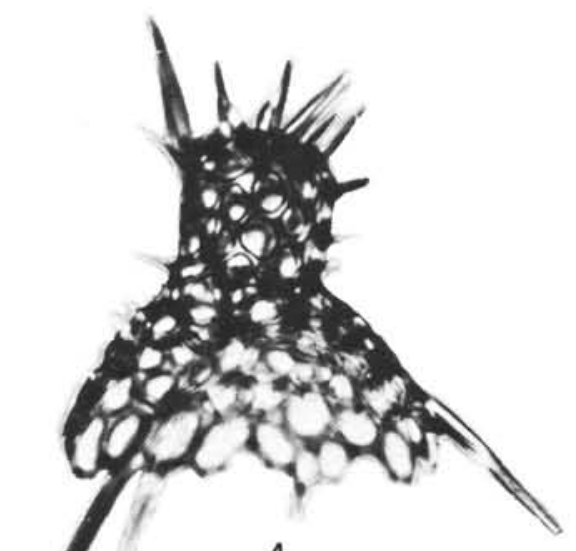

4

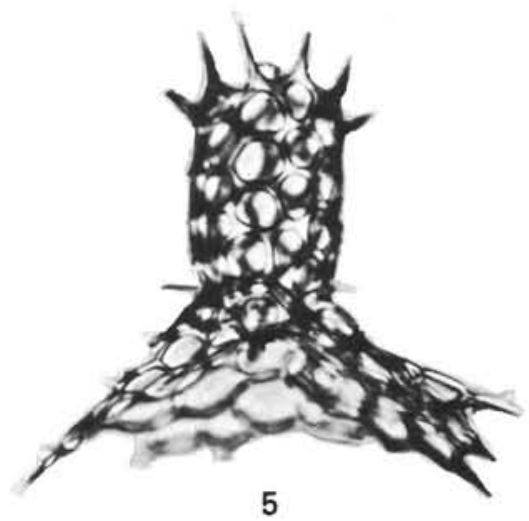

5
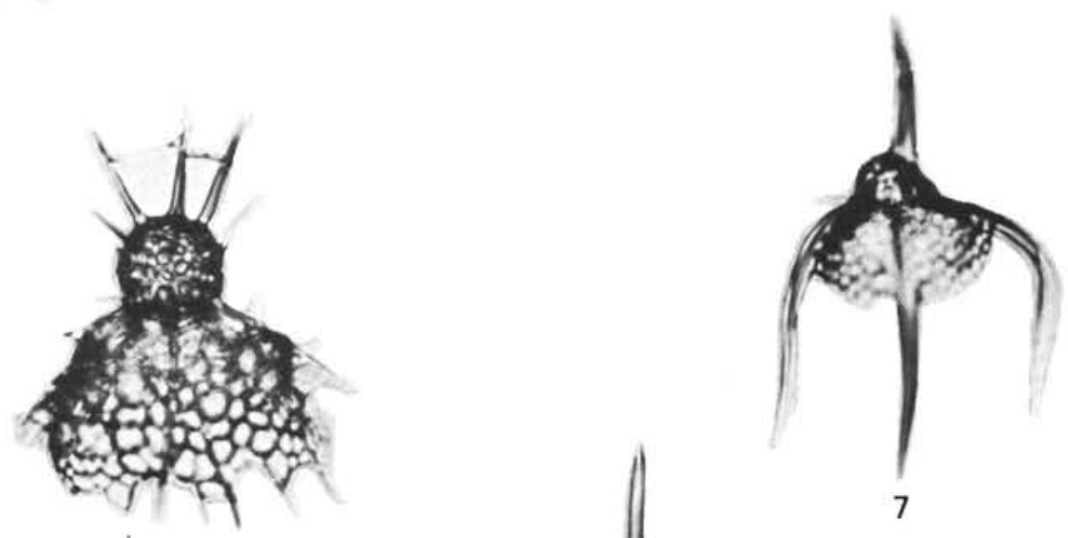

6

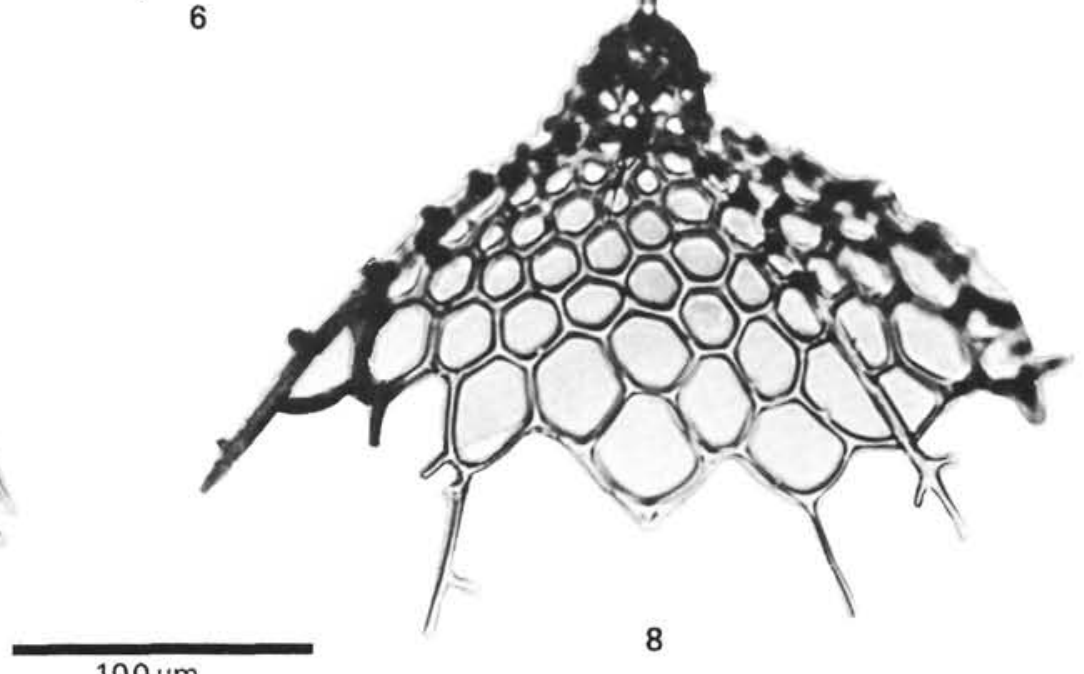

$100 \mu \mathrm{m}$

Plate 8. Radiolarians from Holocene sediments in the Gulf of California. (Scale bar equals $100 \mu \mathrm{m}$.) 1-3. Helotholus histricosa Jorgensen group, (1) VS-R-115a, 1-3 cm, S47/0. Left lateral view. Benson, 1966, pl. 31, fig. 6, (2) VS-R-151b, 1-3 cm, V31/4. Left lateral view. Benson, 1966, pl. 31, fig. 4, (3) VS-R-60a, 3-5 cm, E9/3. Right lateral view. Benson, 1966, pl. 31, fig. 8. 4. Dictyophimus sp. cf. D. tripus Haeckel. VS-R-81a, 1-3 cm, V49/1. Right lateral view. Benson, 1966, pl. 25, fig. 3. 5. Amphiplecta cylindrocephala Dumitrica. VS-R-60a, 3-5 cm, U9/0. Right lateral view. Benson, 1966, pl. 32, fig. 2. 6. Arachnocorys umbellifera Haeckel. VS-R-64a, 1-3 cm, Y20/4. Ventral view. Benson, 1966, pl. 24, fig. 21. 7. Dictyophimus platycephalus Haeckel. VS-R-60b, 3-5 cm, L23/0. Left lateral view. Benson, 1966, pl. 25, fig. 7. 8. Lampromitra quadricuspis Haeckel. VS-R-56a, 1-3 cm, D33/1. Ventral view, Benson, 1966, pl. 31, fig. 1.

See Note, Plate 4. 

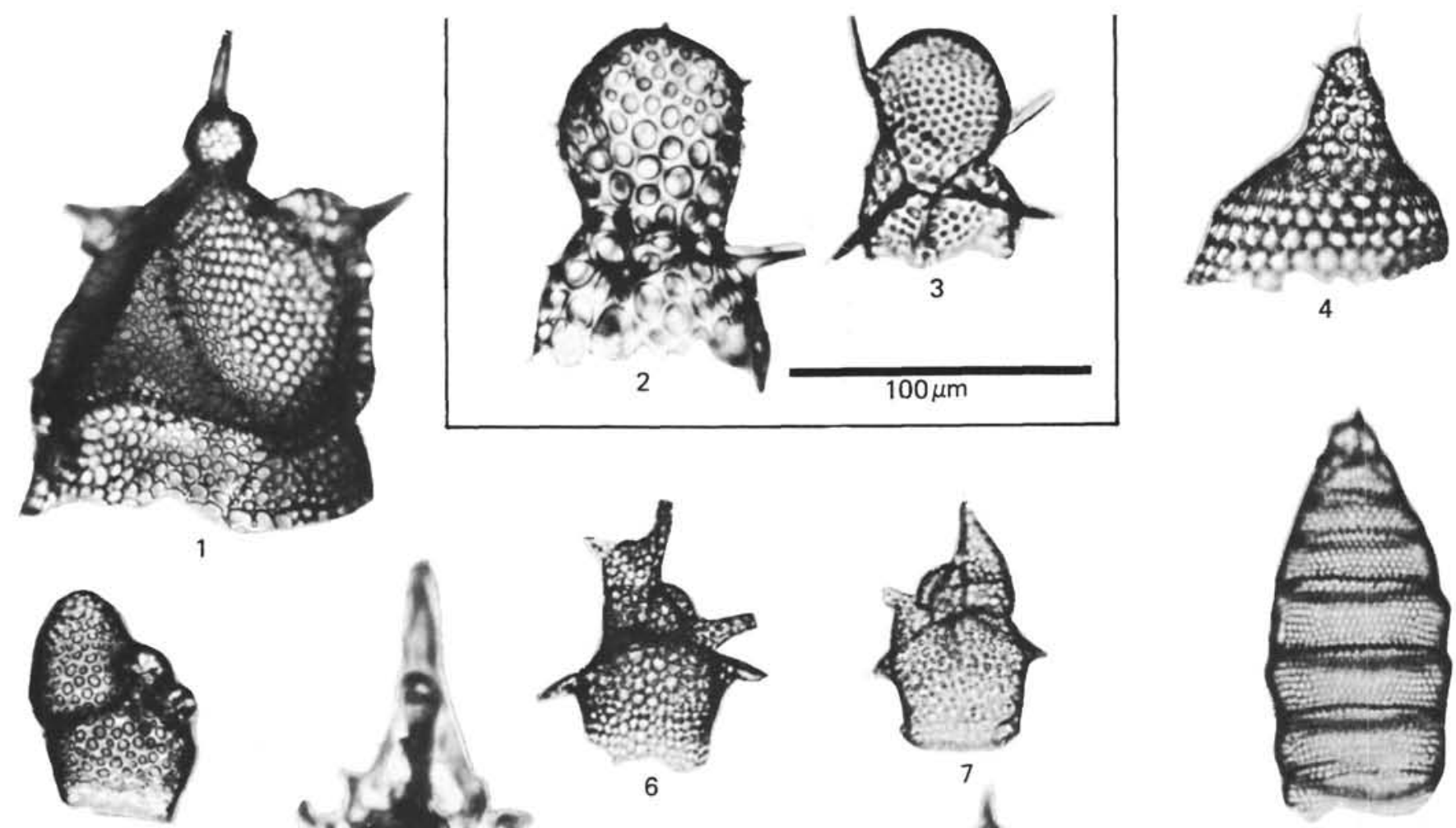

1

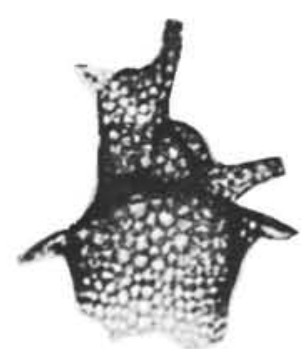

6

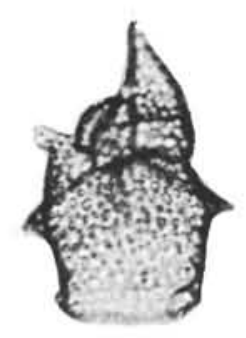

7

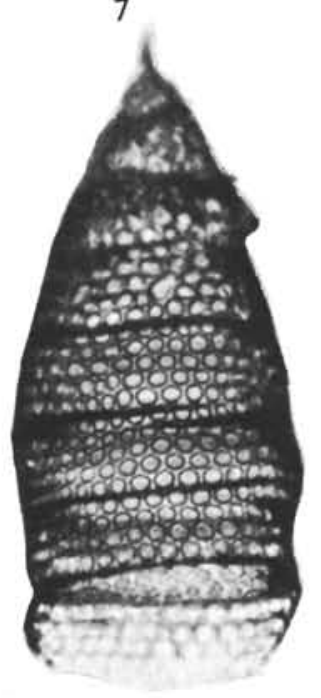

10

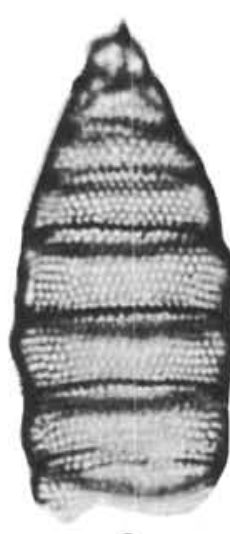

9

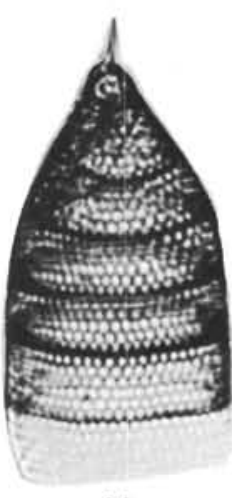

11

$100 \mu \mathrm{m}$

Plate 9. Radiolarians from Holocene sediments in the Gulf of California. (Scale bars equal $100 \mu \mathrm{m}$.) 1. Lipmanella tribranchiata Dumitrică. VSR-81a, 1-3 cm, G41/1. Doroso-right lateral view. Benson, 1966, pl. 28, fig. 11. 2. Lithomelissa thoracites Haeckel. VS-R-92b, 1-3 cm, E23/0. Ventro-right lateral view. Benson, 1966, pl. 24, fig. 11. 3. Lithomelissa laticeps Jorgensen. VS-R-93b, 1-3 cm, Z46/0. Right lateral view. Benson, 1966, pl. 24, fig. 14. 4. Eucecryphalus(?) sp. VS-R-192b, 1-3 cm, U34/2. Left lateral view. Benson, 1966, pl. 30, fig. 6. 5. Acrobotrissa cribosa Popofsky. VS-R-60a, 3-5 cm, O39/4. Right lateral view. Benson, 1966, pl. 23, fig. 15. 6-7. Acrobotrys sp. cf. A. disolenia Haeckel, (6) VS-R-81a, 1-3 cm, G29/0. Right lateral view. Benson, 1966, pl. 23, fig. 14, (7) VS-R-93b, 1-3 cm, D46/2. Left lateral view. Benson, 1966, pl. 23, fig. 13. 8. Clathromitra pterophormis Haeckel. VS-R-56a, 1-3 cm, E19/2. Left lateral view from below, focus on basal tripodium. Benson, 1966, pl. 26, fig. 4. Figure 9-11. Eucyrtidium(?) hexastichum (Haeckel) group, (9) VS-R-92a, 1-3 cm, D20/0. Benson, 1966, pl. 34, fig. 16, (10) VS-R-60b, 3-5 cm, N45/0. Benson, 1966, pl. 34, fig. 13, (11) VS-R-60b, 3-5 cm, F43/1. Benson, 1966, pl. 34, fig. 15. See Note, Plate 4. 